HU-EP-02/23

SPIN-02/17

ITP-UU-02/26

hep-th/0206038

\title{
Orientifolds of K3 and Calabi-Yau Manifolds with Intersecting D-branes
}

\author{
Ralph Blumenhagen ${ }^{1}$, Volker Braun ${ }^{1}$, Boris Körs ${ }^{2}$, and Dieter Lüst ${ }^{1}$ \\ ${ }^{1}$ Humboldt-Universität zu Berlin, Institut für Physik, \\ Invalidenstrasse 110, 10115 Berlin, Germany \\ e-mail: blumenha, braun, luest@physik.hu-berlin.de \\ 2 Spinoza Institute, Utrecht University, \\ Utrecht, The Netherlands \\ email: kors@phys.uu.nl
}

\begin{abstract}
We investigate orientifolds of type II string theory on K3 and Calabi-Yau 3-folds with intersecting D-branes wrapping special Lagrangian cycles. We determine quite generically the chiral massless spectrum in terms of topological invariants and discuss both orbifold examples and algebraic realizations in detail. Intriguingly, the developed techniques provide an elegant way to figure out the chiral sector of orientifold models without computing any explicit string partition function. As a new example we derive a non-supersymmetric Standard-like Model from an orientifold of type IIA on the quintic Calabi-Yau 3-fold with wrapped D6-branes. In the case of supersymmetric intersecting brane models on CalabiYau manifolds we discuss the D-term and F-term potentials, the effective gauge couplings and the Green-Schwarz mechanism. The mirror symmetric formulation of this construction is provided within type IIB theory. We finally include a short discussion about the lift of these models from type IIB on K3 to F-theory and from type IIA on Calabi-Yau 3 -folds to M-theory on $G_{2}$ manifolds.
\end{abstract}

$06 / 2002$ 


\section{Introduction}

One of the ultimate goals of string theory is to provide a model from which one might derive the real low energy physics as its effective theory. Of course, we are still far away from a solution to this problem, but recently new models have been devised that qualitatively come fairly close to the Standard Model in many respects. The main new ingredient in these models is that they contain intersecting D-branes and open strings in a consistent manner, which provide simple mechanisms to generate chiral fermions and to break supersymmetry [1-29]. They were therefore called intersecting brane worlds. The main focus has so far been put on orientifolds for which the background geometry is realized as a torus or a toroidal orbifold. This restriction we want to remove in the present paper, and generalize the concept of intersecting brane worlds to generic background geometries of K3 or Calabi-Yau 3-folds. We construct six- and four-dimensional orientifold vacua of type II string theories with D-branes wrapping cycles of middle dimension in the internal compact space. The patterns of their intersections govern the properties of the effective theory, such as its chiral fermion spectrum, the breaking of supersymmetry and the scalar potential generated for the moduli fields.

In brane world models one usually considers D-branes wrapping part of the internal compactification space and filling out the non-compact space-time. It is clear that a single D-brane wrapping a cycle in some compact Calabi-Yau manifold is not a consistent string background of such a type, as the total Ramond-Ramond (RR)-charge has to vanish. One way to remedy the situation is to consider non-compact Calabi-Yau manifolds allowing the RR-flux to escape to infinity. This option is only applicable to local models of gauge theories, as the gravitational excitations on the internal space will in general not decouple from the effective four-dimensional physics. Another option within type II string theory is to use D-branes together with their anti-branes, which will allow for configurations with vanishing RR-charge, however at the price of breaking supersymmetry [30]. Since for a supersymmetric model not only the RR-charges must add up to zero but also the overall tension of the branes must vanish, one must introduce objects with negative tension to compensate for the branes. Such objects are provided by orientifold planes, so that the natural arena for compact models are orientifolds of type II string theory on Calabi-Yau manifolds.

Apparently, in such models we have to deal with the description of D-branes wrapping internal cycles of the K3 respectively the Calabi-Yau manifold. Independent from more 
phenomenological considerations, the physics of D-branes wrapped on supersymmetric cycles in some Calabi-Yau manifold was studied in detail during the last few years [31-45]. As a very striking result the notion of mirror symmetry has been shown to generalize to such open string models, in simple cases allowing to compute the corrections to the $\mathcal{N}=1$ superpotential due to world-sheet disc instantons by utilizing the perturbative tree level result of the mirror configuration. In principle these techniques should find a natural application in the far more complicated models we are going to discuss in this paper. Thus, in generalizing the orientifold construction from the singular orbifold backgrounds to generic Calabi-Yau spaces, we hope to provide opportunities for contact between the two fields of study in the future.

The rather simple toroidal and orbifold intersecting brane worlds models have been of interest during the last two years for their ability to produce phenomenologically appealing string vacua in a simple way. They allow for a bottom-up approach to search for both supersymmetric and non-supersymmetric stringy realizations of the Standard Model or grand unified extensions thereof [8, 11, 12, 13, 25]. One starts with a conventional orientifold model, defined by taking the quotient of a type II string theory by a group of symmetries of the background and the world sheet parity $\Omega$. For simplicity, one can restrict to the case that the orientifold planes just wrap middle dimensional homological cycles of the underlying manifold. It is then possible to introduce generic intersecting D-branes wrapping middle class homological cycles, as well, in a way that the total RR-charge cancels among the two. At the intersection of any such D-branes chiral fermions are localized [46]. It was shown that one can actually maintain supersymmetry in particular models of this kind [2, [7, 12, 13], but generically it will be broken. If the supersymmetric models are perturbed, a scalar potential will be generated in the form of an F- or D-term and tachyons can arise. The latter is not automatic and can also be avoided. The scalar potential then freezes some of the Kähler and complex structure moduli. This phenomenon of moduli stabilization can, of course, also be observed in non-supersymmetric compactifications [11,20,24].

It will turn out that a major fraction of the usual orientifold models studied extensively in six and four uncompactified space-time dimensions can be considered as a small subclass of the orientifolds with intersecting branes. The standard compactifications considered in the literature are recovered from the more general class of intersecting brane models by placing the D-branes parallel to the orientifold planes. 
To appreciate some of the results we will derive in this paper let us shortly review, how the CFT construction of an orientifold model on a toroidal orbifold background proceeds technically. ${ }^{1}$ One considers a quotient of a type II string theory on a four- or sixdimensional torus by a finite group which not only contains discrete symmetries but also the world-sheet parity transformation $\Omega$. In order to derive the RR-charge cancellation conditions one needs to compute the Klein bottle amplitude explicitly in order to extract the infra-red divergences in the tree-channel. They are due to the exchange of massless closed string modes, which couple to the RR charge of the orientifold planes. Next one has to add appropriate open string sectors, D-branes, into the theory to cancel the divergences via the interference of the annulus and the Möbius strip amplitudes with the Klein bottle. This means that the RR-charges of the orientifold planes and the D-branes add up to zero. Due to the fact that the coupling of the massless RR closed string modes to the orientifold planes and D-branes is of a topological nature, the conditions that derive from the cancellation requirement only restrict some discrete parameters of the model, like the number of D-branes. But usually, some freedom remains in the construction, for instance in the action of the discrete symmetries on the Chan-Paton factors. Utilizing this information and fixing the arbitrariness by some choice one is finally able to compute the massless spectrum. It is not obvious that the resulting spectrum is given in terms of topological invariants of the D-brane geometry, as it involves a projection in the Chan-Paton gauge bundle on their world volume and the latter seems to depend on the details of the model.

However, from other string models we are used to the fact that the spectrum of chiral fermions is determined entirely by the topology of the compact manifold. For instance, the number of generations in compactifications of the heterotic string on a Calabi-Yau manifold is determined by its Euler characteristic. In the same way, when toroidal intersecting brane models of this type were first considered in [1], it was shown by explicit world sheet CFT computations that the chiral massless spectrum is determined by the topological intersection numbers of the homological cycles the D-branes are wrapping on. Since this relation between chiral fermions and topology is manifest in the anomalies, we expect a similar result to hold in any case. More concretely, one expects that for orientifold models on smooth K3 and Calabi-Yau 3-folds the chiral fermion spectrum should be given by topological invariants of the compactification space, of the submanifolds wrapped by the orientifold planes and the D-branes, and of the gauge bundles on the respective D-branes.

1 For a recent and exhaustive review on open strings and orientifold constructions see [47]. 
By restricting the branes and planes to preserve supersymmetry in the weak sense, that is any single D-brane preserves some supersymmetry, but not all branes and planes necessarily the same, one can simplify the situation considerably (see also [19,22]). The branes then have to be wrapped on certain calibrated cycles and the gauge bundles on their world volume need to be flat. This restriction is natural in the orientifold models which we shall be considering, and will only be relaxed in the non-supersymmetric Standard Model on the quintic in section (7.6). In particular, it allows to write explicit formulas for the chiral fermion spectra. We will see that the reason, why this has not been apparent in most of the orientifold models studied so far, is, that the actual computation has been performed at a singular point in moduli space, where certain cycles have shrunken to zero size.

Concretely, the spectrum of chiral fermions is always given by the topological intersection numbers of the cycles the D-branes are wrapped on. We consider it the main result of this paper to make this explicit and test the resulting spectra for their consistency with a large number of examples. In order to make a comparison to singular orbifold models one first has to perform a blow-up of their singularities and only then compute the intersection numbers on the resulting smooth manifold. We will show that the computations for the orientifold on the blown-up K3 or on Calabi-Yau 3-folds agree completely with the chiral spectrum determined at the orbifold point by the lengthy procedure outlined above. For K3 these examples include T-dual versions of the model commonly known as the Gimon-Polchinski model [48] (even though it was first constructed by Bianchi and Sagnotti [49]) and those studied by Gimon-Johnson [50]. Thus, the developments of this paper can be considered technically as an alternative route towards the determination of the phenomenologically important chiral massless spectrum avoiding the computation of explicit string partition functions.

This paper is organized as follows. In section 2 we introduce the general set-up of intersecting brane worlds on orientifolds of smooth K3 and Calabi-Yau 3-folds. In section 3 we discuss K3 compactifications and present the generic result for the chiral massless spectrum expressed in terms of topological intersection numbers of 2-cycles in the K3. Anomaly cancellation in six dimensions yields a non-trivial constraint on the selfintersection number of the orientifold O7-planes for which we also give a mathematical proof. In section 4 we revisit a number of six-dimensional orientifold models studied so far in the literature and show that the chiral spectra computed by utilizing our novel prescription for the blown-up orbifolds agree with the spectra obtained at the orbifold point. The discussed examples include the original orientifold models of Gimon-Johnson, 
the orientifolds on orbifolds of K3 studied in [51,52,53,54 and a model on the quartic. We study both non-supersymmetric and supersymmetric models. For specific supersymmetric orbifold models we also discuss the lift to F-theory on an elliptically fibered Calabi-Yau threefold. Finally, we show that the compactification of F-theory on Voisin-Borcea CalabiYau 3-folds is consistent with the proposed chiral spectrum, as well. In section 5 we apply our methods to orientifolds on smooth Calabi-Yau 3-folds. Again the chiral matter content is given by the homological intersection numbers of the D6-branes respectively O6-planes. Section 6 is devoted to a detailed discussion of the generalized Green-Schwarz mechanism in these models. In section 7 we present some blown-up orbifold examples and construct on the Quintic Calabi-Yau manifold a non-supersymmetric Standard like Model. We continue in section 8 with the application of known results on the physics of D-branes wrapping special Lagrangian cycles in Calabi-Yau manifolds to supersymmetric intersecting brane worlds. This includes the generation of F-term and D-term potentials as well as a discussion of the gauge couplings. An equivalent mirror symmetric formulation for these set-ups is discussed in section 9 and finally in section 10 we comment on the lift of these configurations to M-theory and the geometric interpretation of their phase transitions. Finally, section 11 contains our conclusions.

\section{Intersecting brane worlds}

The models we are going to study all start from a supersymmetric type II compactification, either of type IIB on a K3 surface or of type IIA on a Calabi-Yau 3-fold, which admits an involution $\bar{\sigma}$ of order 2 . For the most part of the paper, we will want it to be the complex conjugation,

$$
\bar{\sigma}: z_{i} \mapsto \bar{z}_{i}, i=1, \ldots, d
$$

in local coordinates. It is anti-holomorphic in the sense that it takes the holomorphic $d$-form to its conjugate. This geometric involution is then combined with the world sheet parity $\Omega$. Dividing out by $\Omega \bar{\sigma}$ leads to the orientifold backgrounds

$$
\mathcal{X}=\mathbb{R}^{9-2 d, 1} \times \frac{\mathcal{M}^{2 d}}{\Omega \bar{\sigma}}
$$

we are going to study. Of course, these models have some relations to type I string compactifications, which we are also going to use on occasion. Our examples for $\mathcal{M}^{2 d}$ will 
involve K3 and Calabi-Yau orbifolds as well as algebraic models such as the quartic in $\mathbb{C P}^{3}$ and the quintic in $\mathbb{C P}^{4}$.

As is well known, the fixed locus $\operatorname{Fix}(\bar{\sigma})$ defines the location of orientifold Oq-planes of dimension $q+1=10-d$, which takes values $q+1=8,7$ in the cases at hand. They extend into $d$ internal directions and fill out the space-time. Just by the definition of $\bar{\sigma}$, $\operatorname{Fix}(\bar{\sigma})$ is a special Lagrangian (sLag) submanifold of the internal space. Define locally the holomorphic $d$-form $\Omega_{d}$ and the Kähler form $J$ by

$$
\Omega_{d}=d z_{1} \wedge \ldots \wedge d z_{d}, \quad J=i \sum_{i=1}^{d} d z_{i} \wedge d \bar{z}_{i}
$$

From $\bar{\sigma}\left(\Omega_{d}\right)=\bar{\Omega}_{d}$ and $\bar{\sigma}(J)=-J$ it follows that

$$
\left.\Im\left(\Omega_{d}\right)\right|_{\operatorname{Fix}(\bar{\sigma})}=0,\left.\quad J\right|_{\operatorname{Fix}(\bar{\sigma})}=0 .
$$

Furthermore the holomorphic $d$-form $\Omega_{d}$ satisfies

$$
-i^{d} \int_{\mathcal{M}} \Omega_{d} \wedge \bar{\Omega}_{d}=\operatorname{Vol}\left(\mathcal{M}^{2 d}\right)
$$

and this then determines

$$
\left.\Re\left(\Omega_{d}\right)\right|_{\operatorname{Fix}(\bar{\sigma})}=\left.d \operatorname{vol}\right|_{\operatorname{Fix}(\bar{\sigma})},
$$

which means, $\operatorname{Fix}(\bar{\sigma})$ is calibrated with respect to $\Re\left(\Omega_{d}\right)$. For future reference we also introduce the normalized holomorphic $d$-form

$$
\widehat{\Omega}_{d}=\frac{1}{\sqrt{\operatorname{Vol}\left(\mathcal{M}^{2 d}\right)}} \Omega_{d} .
$$

\subsection{Tadpole conditions and the scalar potential}

In the Klein bottle amplitude, which is, of course, only computable at very special points in the moduli space where a CFT description is available, the orientifold planes introduce IR divergences which have to be canceled by similar contributions from $\mathrm{D} q$ branes. The RR tadpole cancellation condition can be deduced on very general grounds from the Chern-Simons action for the Dq-branes [55-59]

$$
\mathcal{S}_{\mathrm{CS}}^{(\mathrm{D} q)}=\mu_{q} \int_{\mathrm{D} q} \operatorname{ch}(\mathcal{F}) \wedge \sqrt{\frac{\hat{\mathcal{A}}\left(\mathcal{R}_{T}\right)}{\hat{\mathcal{A}}\left(\mathcal{R}_{N}\right)}} \wedge \sum_{p} C_{p}
$$


and the orientifold planes

$$
\mathcal{S}_{\mathrm{CS}}^{(\mathrm{O} q)}=Q_{q} \mu_{q} \int_{\mathrm{O} q} \sqrt{\frac{\hat{\mathcal{L}}\left(\mathcal{R}_{T} / 4\right)}{\hat{\mathcal{L}}\left(\mathcal{R}_{N} / 4\right)}} \wedge \sum_{p} C_{p} .
$$

Here the RR-charge quantum is given by

$$
\mu_{q}=\frac{1}{(2 \pi)^{q}}\left(\alpha^{\prime}\right)^{-\frac{q+1}{2}}
$$

and the sum is over all RR-forms $C_{p}$ in the respective theory. The relative charge of the orientifold planes is given by $Q_{q}=-2^{q-4}$. The Chern character, Dirac genus and the Hirzebruch polynomial are defined via

$$
\begin{aligned}
\operatorname{ch}(\mathcal{F}) & =\operatorname{Tr} e^{i \mathcal{F} / 2 \pi}, \\
\hat{\mathcal{A}}(\mathcal{R}) & =1-\frac{p_{1}(\mathcal{R})}{24}+\cdots=1+\hat{\mathcal{A}}_{4}(\mathcal{R})+\cdots, \\
\hat{\mathcal{L}}(\mathcal{R} / 4) & =1+\frac{p_{1}(\mathcal{R})}{48}+\cdots=1+\hat{\mathcal{L}}_{4}(\mathcal{R} / 4)+\cdots .
\end{aligned}
$$

Moreover, $\mathcal{R}_{T}$ and $\mathcal{R}_{N}$ denote the pullbacks of the curvature two-forms of the tangent and normal bundle on the brane. The physical gauge fields and curvatures are related to the skew-hermitian ones in (2.8) by $\mathcal{F}=-4 i \pi^{2} \alpha^{\prime} F$ and $\mathcal{R}=-4 i \pi^{2} \alpha^{\prime} R$. In all the models we shall be considering, the D-branes will be wrapping on calibrated sLag cycles of real dimension 2 or 3 . Since the supersymmetry condition on such a sLag cycles states that the gauge bundle has to be flat, we get the simplification $\left.\operatorname{ch}(\mathcal{F})\right|_{\mathrm{D} q}=\left.\operatorname{ch}_{0}(\mathcal{F})\right|_{\mathrm{D} q}=\operatorname{rk}(\mathcal{F})$. Moreover, the pull-back of the Pontryagin class $p_{1}(\mathcal{R})$ onto these lower dimensional branes reduces to $\left.p_{1}(\mathcal{R})\right|_{\mathrm{D} q}=0$. The only contribution in the CS-term (2.8) now comes from $C_{q+1}$.

Let us denote the homology class of the fixed point set of the orientifold projection by $\pi_{\mathrm{O} q}=[\operatorname{Fix}(\bar{\sigma})] \in H_{d}\left(\mathcal{M}^{2 d}\right)$. Similarly we denote the homology class a $\mathrm{D} q_{a}$-brane is wrapping around by $\pi_{a}$. In general, such a $\pi_{a}$ is not invariant under the $\Omega \bar{\sigma}$ projection but is mapped to a different cycle $\pi_{a}^{\prime}$. Therefore, one is forced to introduce a D-brane wrapped on that cycle, too. The part of the supergravity Lagrangian where the RR-field $C_{q+1}$ appears reads

$$
\begin{aligned}
\mathcal{S}=-\frac{1}{4 \kappa^{2}} \int_{\mathcal{X}} d C_{q+1} \wedge \star d C_{q+1}+\mu_{q} \sum_{a} N_{a} \int_{\mathbb{R}^{9-2 d, 1} \times \pi_{a}} C_{q+1} \\
+\mu_{q} \sum_{a} N_{a} \int_{\mathbb{R}^{9-2 d, 1} \times \pi_{a}^{\prime}} C_{q+1}+\mu_{q} Q_{q} \int_{\mathbb{R}^{9-2 d, 1} \times \pi_{\mathrm{O} q}} C_{q+1},
\end{aligned}
$$


where the ten-dimensional gravitational coupling is $\kappa^{2}=\frac{1}{2}(2 \pi)^{7}\left(\alpha^{\prime}\right)^{4}$. The resulting equation of motion for the RR field strength $G_{q+2}=d C_{q+1}$ is

$$
\frac{1}{\kappa^{2}} d \star G_{q+2}=\mu_{q} \sum_{a} N_{a} \delta\left(\pi_{a}\right)+\mu_{q} \sum_{a} N_{a} \delta\left(\pi_{a}^{\prime}\right)+\mu_{q} Q_{q} \delta\left(\pi_{\mathrm{O} q}\right)
$$

where $\delta\left(\pi_{a}\right)$ denotes the Poincaré dual form of $\pi_{a}$. Since the left hand side in (2.13) is exact, the RR-tadpole cancellation condition becomes in homology

$$
\sum_{a} N_{a}\left(\pi_{a}+\pi_{a}^{\prime}\right)+Q_{q} \pi_{O q}=0
$$

For D-branes not lying directly on top of the orientifold plane, any stack of $N_{a}$ branes will then support one factor of the total gauge group

$$
G=\prod_{a} U\left(N_{a}\right)
$$

Only if a stack of D-branes is located within the fixed locus of $\bar{\sigma}$, also $S O\left(N_{a}\right)$ or $S p\left(N_{a}\right)$ gauge groups can occur. Since this case is less generic, we will restrict our attention to unitary gauge groups for the most part of this paper. Compared to type I string theory with a gauge groups $S O(32)$ in ten dimensions 2.15) can be interpreted as a particular pattern of gauge symmetry breaking which involves a reduction of the rank of the gauge group, as well. In principle, the tadpole condition (2.14) involves as many linear relations as there are independent cycles in $H_{d}\left(\mathcal{M}^{2 d}, \mathbb{R}\right)$. But, of course, the action of $\bar{\sigma}$ on $\mathcal{M}^{2 d}$ also induces an action $[\bar{\sigma}]$ on the homology and cohomology. In particular, $[\bar{\sigma}]$ swaps $H^{r, s}$ and $H^{s, r}$, and therefore for 3 -folds the number of conditions is halved in any case.

Similarly one can generally determine the disc level NSNS tadpoles. These result from integrating the Dirac-Born-Infeld effective action [60,61]

$$
\mathcal{S}_{\mathrm{DBI}}=-T_{q} \int_{\mathcal{M}_{q+1}} d^{q+1} x e^{-\phi_{10}} \sqrt{g_{q+1}}
$$

of the $\mathrm{D} q$-branes with the tension given by $T_{q}=\mu_{q}$. The action (2.16) is proportional to the volume of the $\mathrm{D} q$-branes respectively the $\mathrm{O} q$-plane, so that the disc level scalar potential reads

$$
\mathcal{V}=T_{q} \frac{e^{-\phi_{10-2 d}}}{\sqrt{\operatorname{Vol}\left(\mathcal{M}^{2 \mathrm{~d}}\right)}}\left(\sum_{a} N_{a}\left(\operatorname{Vol}\left(\mathrm{D} q_{a}\right)+\operatorname{Vol}\left(\mathrm{D} q_{a}^{\prime}\right)\right)+Q_{q} \operatorname{Vol}(\mathrm{O} q)\right)
$$


Generically, this scalar potential is non-vanishing reflecting the fact that intersecting branes in general break supersymmetry. Non-supersymmetric compactifications can still be phenomenologically relevant in the context of large transverse volume compactifications [62:63]. Note, that obstructions to these scenarios met with toroidal models [1] are no longer present in the more general backgrounds we are going to consider. But since we are eventually interested in supersymmetric intersecting brane worlds, let us specialize to D-branes wrapping sLag cycles of K3 respectively Calabi-Yau 3-folds for the remainder of this section. These can in principle be calibrated with respect to different $d$-forms $\Re\left(e^{i \theta} \Omega_{d}\right)$, differing by a constant phase factor, which means that each of them preserves supersymmetry, but not necessarily all of them the same. Effectively, supersymmetry can be broken completely. For D-branes wrapping sLag cycles the scalar potential (2.17) only depends on the complex structure moduli, as in this case the scalar potential gets simplified to

$$
\mathcal{V}=T_{q} e^{-\phi_{10-2 d}}\left(\sum_{a} N_{a}\left|\int_{\pi_{a}} \widehat{\Omega}_{d}\right|+\sum_{a} N_{a}\left|\int_{\pi_{a}^{\prime}} \widehat{\Omega}_{d}\right|+Q_{q}\left|\int_{\pi_{\mathrm{O} q}} \widehat{\Omega}_{d}\right|\right) .
$$

Since $\widehat{\Omega}_{d}$ is closed, the integrals only depend on the homology class of the world volume of the branes and planes. Supersymmetric models in this context are then defined by requiring that any single $\mathrm{D} q_{a}$-brane conserves the same supersymmetries as the orientifold plane. In other words, the cycles the $\mathrm{D} q$-branes wrap on must be calibrated with respect to the same calibration form $\Re\left(\widehat{\Omega}_{d}\right)$ as the $\mathrm{O} q$-planes. In this particular case we can write (2.18) as

$$
\mathcal{V}=T_{q} e^{-\phi_{10-2 d}}\left(\sum_{a} N_{a} \int_{\pi_{a}+\pi_{a}^{\prime}} \Re\left(\widehat{\Omega}_{d}\right)+Q_{q} \int_{\pi_{\mathrm{O} q}} \Re\left(\widehat{\Omega}_{d}\right)\right) .
$$

Due to the RR-tadpole cancellation condition (2.14) this vanishes, so that the scalar potential is zero, as expected in the supersymmetric situation .

\subsection{Spectra of massless bulk modes}

It is instructive to study the action of $\Omega \bar{\sigma}$ on the cohomology in a bit more detail. While the fixed locus $\operatorname{Fix}(\bar{\sigma}) \subset \mathcal{M}^{2 d}$ may well be empty, the set of $\bar{\sigma}$ invariant d-cycles $\operatorname{Fix}([\bar{\sigma}]) \subset H^{d}\left(\mathcal{M}^{2 d}, \mathbb{R}\right)$, never is, just as $\Re\left(\Omega_{d}\right)$ defines an invariant class in $H^{d}$. Of course, we always have $[\operatorname{Fix}(\bar{\sigma})] \in \operatorname{Fix}([\bar{\sigma}])$. The importance of $[\bar{\sigma}]$ lies in the fact that it already determines completely the low energy spectrum of gravity fields. They arise from the dimensional reduction of the respective type II string theory upon expanding ten-dimensional fields in terms of harmonic forms on $\mathcal{M}^{2 d}$. 
For compactifications of type IIB string theory on K3 the cancellation of the sixdimensional gravitational anomalies links the closed and open string spectra. The rules are fairly simple: Any harmonic $(1,1)$-form $\omega_{i}, i=1, \ldots, h^{(1,1)}$, gives rise to a six-dimensional $\mathcal{N}=(0,1)$ hypermultiplet with four scalars from the reduction of the metric and the $\mathrm{RR}$ 2 -form in addition to a tensormultiplet containing the self-dual six-dimensional tensors that descend from the self-dual RR 4-form and a scalar from the reduction of the NSNS 2form. Under the world-sheet parity transformation only the $(1,1)$ forms are anti-invariant $\omega_{i} \mapsto-\omega_{i}$ implying the following projections for massless closed string modes in the $\Omega \bar{\sigma}$ orientifold. If under $\bar{\sigma}$ the 2 -form $\omega_{i}$ is anti-invariant we get a massless hypermultiplet and, if the 2 -form $\omega_{i}$ is invariant, a massless tensor-multiplet survives the projection. Since one tensor is part of the supergravity multiplet, in summary we can write

$$
n_{\mathrm{H}}+n_{\mathrm{T}}=21, \quad n_{\mathrm{T}}=\operatorname{dim}\left(\operatorname{Fix}([\bar{\sigma}]) \cap H^{(1,1)}\left(\mathcal{M}^{2 d}\right)\right)
$$

The extra tensormultiplets enter the anomaly cancellation condition for the irreducible $R^{4}$ term and need to be balanced by chiral matter in the open string spectrum. We postpone the concrete computation of anomalies until we specialize to particular dimensions in the following sections. Very generally, it is determined by the number of intersections of the D-branes on the internal space. Roughly speaking, any single intersection point of two branes supports a single chiral fermion in the effective theory, which transforms as a bifundamental under the two respective gauge groups on the branes. For flat branes this is confirmed by the quantization of open strings with the appropriate boundary conditions. In a non-trivial background one can employ geometrical methods at large volume, where the classical geometry is valid. Then, chiral fermions are identified as zero-modes of the Dirac operator. By the Atiyah-Singer index theorem, these are related to topological invariants of the background manifold and the gauge bundle on top.

For Calabi-Yau threefolds the story is very similar. The bulk supersymmetry is reduced by the $\Omega \bar{\sigma}$ projection from $\mathcal{N}=2$ to $\mathcal{N}=1$. This implies that all bulk $\mathcal{N}=2$ superfields are truncated to $\mathcal{N}=1$ superfields by the $\Omega \bar{\sigma}$ projection. Before the projection there were $h^{(1,1)}$ abelian vector multiplets and $h^{(2,1)}$ hypermultiplets. On the one hand the $h^{(1,1)}$ vector multiplets consist of 1 scalar coming from the dimensional reduction of the gravity field (the Kähler modulus), another scalar resulting from the reduction of the NSNS 2-form and a four-dimensional vector from the reduction of the RR 3-form along the 2-cycle. Similar to the K3 case, if the $(1,1)$ form is invariant under $\Omega \bar{\sigma}$ an $\mathcal{N}=1$ 
chiral multiplet survives the projection and if it is anti-invariant we get an $\mathcal{N}=1$ vector multiplet. Note, that the surviving chiral multiplets still contain the complexified Kähler moduli.

On the other hand the four scalars of the $h^{(2,1)}$ hypermultiplets contain 2 scalars from the ten-dimensional gravity field (the complex structure moduli) equipped with 2 scalars arising from the dimensional reduction of the $\mathrm{RR} 3$-form along the two associated 3-cycles referring to $H^{2,1}\left(\mathcal{M}^{3}\right)$ and $H^{1,2}\left(\mathcal{M}^{3}\right)$. Under the $\Omega \bar{\sigma}$ projection one of the two components of the complex structure is divided out and moreover one linear combination of the RR scalars survives, so that the former quaternionic complex structure moduli space gets reduced to a complex moduli space of dimension $h^{(2,1)}$.

\subsection{Orbifold models}

Something more can be said in the cases where $\mathcal{M}^{2 d}$ is a toroidal orbifold of K3 or a Calabi-Yau 3-fold. First of all, $\operatorname{Fix}(\bar{\sigma})$ can be determined rather generally. Let us restrict to orbifold groups $\mathbb{Z}_{N}=\left\{\Theta, \Theta^{2}, \ldots, 1\right\}$, that act crystallographically on $T^{2 d}$ which we assume to be a direct product of two-dimensional tori $T_{I}^{2}, I=1, \ldots, d$. In chapter 7.2 , we shall actually also discuss the orbifold $T^{6} / \mathbb{Z}_{2} \times \mathbb{Z}_{2}$, but the generalizations required are fairly obvious. When choosing local coordinates $z_{I}$, the factorization implies a diagonal complex structure $\tau^{I J}$,

$$
d z_{I}=d x_{I}+\sum_{J=1}^{d} \tau^{I J} d y_{J}=d x_{I}+\tau^{I} d y_{I}, \quad z_{I} \equiv z_{I}+1, \quad z_{I} \equiv z_{I}+\tau^{I}
$$

These coordinates diagonalize the orbifold action by

$$
\Theta z_{I}=e^{2 \pi i v_{I}} z_{I}, \quad \sum_{I=1}^{d} v_{I}=0
$$

and $\bar{\sigma}$ reflects $y_{I}$. The orbifold action preserves the supersymmetry generators that satisfy

$$
\Theta \epsilon= \pm \epsilon
$$

The entire orientifold group is now generated by $\Omega \bar{\sigma}$ and $\Theta$. By using

$$
\Theta^{1 / 2} \bar{\sigma} \Theta^{-1 / 2}=\Theta \bar{\sigma}
$$


it is evident that the fixed locus of $\bar{\sigma}$ on the quotient space can be understood as the orbit of its fixed locus $\left.\operatorname{Fix}(\bar{\sigma})\right|_{T^{2 d}}$ on the tori, the product of the $d$ real circles, in addition to the orbit of $\left.\operatorname{Fix}(\Theta \bar{\sigma})\right|_{T^{2 d}}$. Altogether we can write

$$
\operatorname{Fix}(\bar{\sigma})=\bigcup_{i=0}^{N-1} \Theta^{i}\left(\left.\operatorname{Fix}(\bar{\sigma})\right|_{T^{2 d}}\right) \cup \Theta^{i}\left(\left.\operatorname{Fix}(\Theta \bar{\sigma})\right|_{T^{2 d}}\right)
$$

Since $\Theta^{1 / 2} \in \mathbb{Z}_{N}$ if and only if $N \in 2 \mathbb{Z}+1$, the two orbits are identical in these cases.

The condition that a pair of two flat $\mathrm{D} q$-branes preserves any supersymmetry is deduced from the Killing spinor equations 46]

$$
\Gamma_{0 \cdots q} \epsilon= \pm \tilde{\epsilon}, \quad \Xi \Gamma_{0 \cdots q} \Xi^{-1} \epsilon= \pm \tilde{\epsilon}
$$

which imply

$$
\Xi^{2} \epsilon= \pm \epsilon
$$

Here, $\Xi$ denotes the relative rotation of the two branes. It is evident, that $\mathrm{D} q$-branes in an orbifold trivially preserve supersymmetry as long as they are related by rotations $\Xi^{2}=\Theta$, but (2.23) and (2.27) have more interesting common solutions. For the relative angles $\varphi_{I}$ of any one of the two branes with respect to the orientifold plane the condition (2.27) requires

$$
\sum_{I=1}^{d} \delta_{I} \varphi_{I}=\theta
$$

with some fixed angle $\theta$ and arbitrary signs $\delta_{I}= \pm 1$. If we further demand that they preserve the same supersymmetry as the orientifold $\mathrm{O} q$-plane, we have to impose

$$
\sum_{I=1}^{d} \varphi_{I}=0
$$

This distinction corresponds to branes wrapping general sLag cycles calibrated by $\Re\left(e^{i \theta} \Omega_{d}\right)$, respectively branes calibrated by $\Re\left(\Omega_{d}\right)$. The relative signs $\delta_{I}=1$ of the $d$ angles in (2.29) are dictated by those of the $v_{I}$ in (2.22) via (2.23).

Furthermore, the action of $[\bar{\sigma}]$ on the cohomology classes of degree $d$ can be classified in orbifold models. For the case of $\mathrm{K} 3$ it is evident that $\bar{\sigma}$ leaves only the two classes represented by

$$
d z_{1} \wedge d z_{2}+d \bar{z}_{1} \wedge d \bar{z}_{2}, \quad d z_{1} \wedge d \bar{z}_{2}+d \bar{z}_{1} \wedge d z_{2}
$$


invariant among those classes of 2-forms, which descend from the torus. Only the first one, in fact $\Omega_{2}+\bar{\Omega}_{2}$, is also invariant under $\Theta$ with the exception of the case $N=2$, where both 2forms are invariant. Cycles that are calibrated with respect to this second $\bar{\sigma}$-invariant form come with a relative sign switch in the angle criterion (2.29) and preserve supersymmetries with the opposite chirality as the orientifold planes. As mentioned already, for the case of a 3-fold, the Dolbeault groups $H^{1,2}$ and $H^{2,1}$ get exchanged by $\bar{\sigma}$ such that one half of the linear combinations are even. All the invariant combinations of $(2,1)$ - and $(1,2)$-forms are of the form

$$
d z_{I} \wedge d z_{J} \wedge d \bar{z}_{K}+d \bar{z}_{I} \wedge d \bar{z}_{J} \wedge d z_{K}
$$

which are invariant under $\Theta$ precisely if $2 v_{K}=0 \bmod \mathbb{Z}$. By examining the table of Calabi-Yau-orbifolds that admit CFT descriptions [64,65], one finds examples that satisfy this condition for any even $N$ and for exactly one $v_{K}$ in each case. Again, using these additional invariant forms as calibrations would introduce different signs into (2.29).

Blowing up any of the orbifold singularities introduces additional cycles. For instance, blowing up an orbifold singularity in a K3 orbifold refers to gluing in $N-1$ copies of a $\mathbb{C P}^{1}$. It may be parameterized by an inhomogeneous coordinate $z_{k}$ as $\left[1, z_{k}\right] \cup[0,1]$, using the index $k$ to label the set of such $\mathbb{C P}^{1}$. Turning on the blow-up mode means adding a term proportional to $d z_{k} \wedge d \bar{z}_{k}$ to the Kähler form $J$. Now $[\bar{\sigma}]$ acts as $(-1) \otimes p$ on these forms, where the $(-1)$ is the intrinsic $\Omega$ reflection of $J$ and $p$ denotes a permutation matrix of order 2. It acts on the labels $k$ and swaps the fixed points of the orbifold generators as a whole. Thus, any doublet of $p$ will give rise to one even and one odd class under $[\bar{\sigma}]$, while any $p$-invariant fixed point will simply be $\bar{\sigma}$-odd. The action of $p$ is of course the place, where the complex structure $\tau^{I}$ starts to play a decisive role, as the relation $z_{I} \equiv z_{I}+\tau^{I}$ determines, if a given fixed point of $\Theta$ is invariant under $\bar{\sigma}$ or not. These data are reflected in the CFT construction of K3 orbifolds by noting that $\Omega \bar{\sigma}$ does not exchange the sectors twisted by $\Theta^{k}$ and $\Theta^{N-k}$ [51]. This is in contrast to the situation in standard type IIB orientifolds with a pure $\Omega$ projection.

\section{Intersecting brane worlds in six dimensions}

In this section we consider intersecting brane worlds on K3 manifolds. We will provide evidence that the massless spectrum of chiral fermions in the effective six-dimensional theory is entirely determined by the topology of the configuration of orientifold planes and D-branes. This result is supported by the partially known orbifold limits of K3 and 
a discussion of its algebraic realization as a quartic in $\mathbb{C P}^{3}$. In addition, we show it to be consistent with the compactification of F-theory on Voisin-Borcea Calabi-Yau 3-folds, which in certain limits are believed to be dual descriptions of orientifold vacua. We also include some considerations on the F-theory lift of the most simple $T^{4} / \mathbb{Z}_{2}$ orbifold. The cancellation of the irreducible gravitational anomaly provides a link between closed and open string spectra and the topology of the orientifold plane. This extra condition is shown to hold in all the models.

\subsection{Chiral spectrum, anomalies and tadpoles}

Let us summarize the set-up: We compactify the type IIB string on a K3 manifold which admits an anti-holomorphic involution $\bar{\sigma}$. Then, we can perform the orientifold projection by $\Omega \bar{\sigma}$, which leads to a model with $\mathcal{N}=(0,1)$ supersymmetry in six dimensions. In general the spectrum of fermions is chiral and the massless closed string spectrum alone does not satisfy the cancellation of the gravitational anomaly. The condition for the vanishing of the coefficient of the irreducible $R^{4}$ term is

$$
n_{\mathrm{H}}-n_{\mathrm{V}}+29 n_{\mathrm{T}}=273
$$

stated in terms of the numbers of hyper-, vector- and tensormultiplets. It puts constraints on the open string spectrum.

The O7-planes are located at the fixed locus $\operatorname{Fix}(\bar{\sigma})$ of $\bar{\sigma}$ and are charged under some of the RR-fields. In terms of the definitions (2.3) they wrap on sLag 2-cycles of the K3 manifold. Their RR-charge must be canceled by stacks of D7-branes wrapping 2-cycles of the K3, as well,

$$
\sum_{a} N_{a}\left(\pi_{a}+\pi_{a}^{\prime}\right)=8 \pi_{\mathrm{O} 7}
$$

The homology group $H_{2}(\mathrm{~K} 3, \mathbb{Z})$ is of dimension $b_{2}=22$ and comes with the intersection form, so it is a Lorentzian lattice of signature $(3,19)$. By choosing a proper basis, it can be put into the standard form

$$
H_{2}(\mathrm{~K} 3, \mathbb{Z}) \cong \Gamma_{1,1} \oplus \Gamma_{1,1} \oplus \Gamma_{1,1} \oplus \Gamma_{\mathrm{E}_{8}} \oplus \Gamma_{\mathrm{E}_{8}}
$$

with $\Gamma_{1,1}$ denoting $2 \times 2$ matrices with entries 0 on the diagonal and 1 off-diagonal, and $\Gamma_{\mathrm{E}_{8}}$ the Cartan matrix of $\mathrm{E}_{8}$. We shall find it an important point to normalize the classes $\pi_{\mathrm{O} 7}$ and $\pi_{a}$ appropriately, when going from $H_{2}(\mathrm{~K} 3, \mathbb{R})$ to $H_{2}(\mathrm{~K} 3, \mathbb{Z})$, which physically refers to the proper Dirac quantization of charges. 
The chiral part of the massless spectrum is expected to be determined by the topology of the configuration due to index theorems. In the case of intersecting branes the chiral matter is supported by open strings localized at the intersection points of the various branes [46]. There are several cases to be distinguished: A self-intersection of any $\mathrm{D} 7_{a}$-brane wrapping the cycle $\pi_{a}$ is never invariant under $\bar{\sigma}$ by our convention, as it is mapped to a self-intersection of the image wrapped on $\pi_{a}^{\prime}$. The excitations at this point therefore do not experience any projection and their Chan-Paton index is an unconstrained $N_{a} \times N_{a}$ matrix. This gives rise to a chiral fermion in the adjoint representation of the $U\left(N_{a}\right)$ gauge group. However, an intersection of a brane with its image can be invariant under $\bar{\sigma}$, which happens precisely if the intersection locus lies within $\operatorname{Fix}(\bar{\sigma})$. The fields at this intersection would then be subject to a projection of the Chan-Paton labels by $\Omega \bar{\sigma}$. Summarizing, intersection points which are invariant under $\Omega \bar{\sigma}$ provide chiral fermions in the anti-symmetric representation of $U\left(N_{a}\right)$, whereas $\Omega \bar{\sigma}$ pairs of intersection points lead to chiral fermions in the anti-symmetric and symmetric representation of the gauge group. Finally, open strings stretched between two different D7-branes always give rise to chiral fermions in the bifundamental representation of the two gauge factors. Together one gets the chiral spectrum of table 1 (the subscripts below the gauge representations denote the transformation properties under the little group $S O(4) \simeq S U(2) \times S U(2)$ in six dimensions).

\begin{tabular}{|c|c|}
\hline Representation & Multiplicity \\
\hline$[\mathbf{A d j}]_{(1,2)}$ & $\pi_{a} \circ \pi_{a}$ \\
\hline$\left[\mathbf{A}_{\mathbf{a}}+\overline{\mathbf{A}}_{\mathbf{a}}\right]_{(1,2)}$ & $\frac{1}{2}\left(\pi_{a} \circ \pi_{a}^{\prime}+\pi_{a} \circ \pi_{\mathrm{O} 7}\right)$ \\
\hline$\left[\mathbf{S}_{\mathbf{a}}+\overline{\mathbf{S}}_{\mathbf{a}}\right]_{(1,2)}$ & $\frac{1}{2}\left(\pi_{a} \circ \pi_{a}^{\prime}-\pi_{a} \circ \pi_{\mathrm{O} 7}\right)$ \\
\hline$\left[\left(\mathbf{N}_{\mathbf{a}}, \mathbf{N}_{\mathbf{b}}\right)+\left(\overline{\mathbf{N}}_{\mathbf{a}}, \overline{\mathbf{N}}_{\mathbf{b}}\right)\right]_{(1,2)}$ & $\pi_{a} \circ \pi_{b}$ \\
\hline$\left[\left(\mathbf{N}_{\mathbf{a}}, \overline{\mathbf{N}}_{\mathbf{b}}\right)+\left(\overline{\mathbf{N}}_{\mathbf{a}}, \mathbf{N}_{\mathbf{b}}\right)\right]_{(1,2)}$ & $\pi_{a} \circ \pi_{b}^{\prime}$ \\
\hline
\end{tabular}

Table 1: $\quad$ Chiral spectrum in $d=6$

Here we denote by $I(x, y)=x \circ y$ the intersection number of $x$ and $y$, i.e. the product in the homology ring. If the intersection number is negative, it is understood that one has to take the opposite chirality. This spectrum will be shown to be completely generic for all orbifold models we are going to consider, as well as for a purely toroidal background. We will also find it consistent with the algebraic model of $\mathrm{K} 3$ as a quartic hypersurface in $\mathbb{C P}^{3}$ 
and with F-theory compactifications on Voisin-Borcea 3-folds. Further, it is formulated entirely in terms of topological quantities invariant under continuous deformations of the moduli. We therefore propose it to be valid throughout the moduli space of the background K3, beyond the singular limits one can actually probe explicitly.

As a consistency check we find that all contributions to the irreducible $F_{a}^{4}$ anomaly coefficients cancel automatically by the tadpole cancellation (2.14). This is necessary as there are no contributions to the gauge anomalies from the closed string sector. For the open string contribution to the gravitational $R^{4}$ anomaly we get

$$
A_{\mathrm{op}}=14 \pi_{\mathrm{O} 7} \circ \pi_{\mathrm{O} 7}
$$

when counted in $N=(0,1)$ supermultiplets. Intriguingly, the anomaly in the open string sector only depends on the intersection number of the O7-plane, which is a closed string quantity. On the other hand, a relation of this kind had to be expected as (3.4) must cancel the contribution $A_{\mathrm{cl}}=273-n_{\mathrm{H}}-29 n_{\mathrm{T}}=28\left(9-n_{\mathrm{T}}\right)$ to the anomaly from the closed string sector. This implies the surprising relation

$$
\pi_{\mathrm{O} 7} \circ \pi_{\mathrm{O} 7}=2\left(9-n_{\mathrm{T}}\right)=\frac{1}{32} \sum_{a, b} N_{a} N_{b}\left(\pi_{a} \circ \pi_{b}+\pi_{a} \circ \pi_{b}^{\prime}\right)
$$

between the self-intersection number of the O7-plane, the number of tensor multiplets and the total intersection of all the D7-branes. At the end of this section we will prove the relation (3.5) from pure mathematical reasoning.

At first glance, the statement that the spectrum in table 1 should hold for each K3 orientifold in general seems to be quite surprising. For special orbifold limits of K3 the chiral massless spectra were obtained after a quite lengthy computation involving the determination of the action of the discrete symmetries on the Chan-Paton factors and the computation of the tadpole cancellation conditions for the various twisted and untwisted sectors, using the explicit one-loop partition function. In the upcoming paragraphs, we will compute the chiral massless spectrum in a number of examples by using table 1 and show that the chiral spectra completely agree with those obtained in the orbifold limits.

A particular class of models consists of those preserving supersymmetry. As was discussed already, supersymmetry implies all the D7-branes to wrap cycles calibrated with respect to $\Re\left(\Omega_{2}\right)$, as do the O7-planes. This already implies the very special form (2.19) for the potential and its vanishing via the tadpole cancellation condition (2.14). The most simple way to preserve supersymmetry is to place the D7-branes right on top of the 
orientifold plane, i.e. set all $\pi_{a}=\pi_{\mathrm{O} 7}$. These models with maximal gauge symmetry have been studied in [48,50,66, 67, 68, 69, 51, 53]. At least at the orbifold points one can still check for supersymmetry, if the D7-branes are not parallel to the orientifold planes, as here the metric is given by the metric on the original torus. Independently, one can explicitly determine the intersection angles of the D7-branes and investigate whether they satisfy (2.29). Since there does not exist a superpotential in six-dimensional field theories with $\mathcal{N}=1$ supersymmetry, supersymmetry breaking can only occur via Fayet-Iliopoulos terms. As we have seen, the scalar potential that arises from the tension of the D7-branes by the reduction of the DBI effective action only depends on the complex structure moduli. For instance, blowing-up the orbifold fixed points should therefore correspond to flat directions and preserve supersymmetry.

\subsection{Mathematical derivation}

Suppose you have a smooth K3 surface $\mathcal{M}$ with smooth antiholomorphic involution $\bar{\sigma}$. The $\bar{\sigma}$-fixed set $\Sigma=\operatorname{Fix}(\bar{\sigma})$ is either empty or a smooth embedded surface (possibly with multiple disconnected components). It is smooth because $\left(T_{p} \mathcal{M}\right)^{\sigma} \simeq T_{p} \Sigma$ varies smoothly for all $p \in \Sigma$. In section 2 we already saw that $\Sigma$ is special Lagrangian. Therefore the complex structure $J: T \mathcal{M} \rightarrow T \mathcal{M}$ maps $T \Sigma$ to $N \Sigma$ and vice versa, so we get an isomorphism

$$
J_{p}: T_{p} \Sigma \simeq N_{p} \Sigma
$$

Now by exponentiation we can associate a small deformation of $\Sigma \subset \mathcal{M}$ to a section of $N \Sigma$, and therefore to a section of $T \Sigma$, i.e. vector fields.

The self-intersection of $\Sigma$ is the intersection with some deformed copy of $\Sigma$. Choose a vector field $\vec{v} \in \Gamma(T \Sigma)$ with simple zeros then

$$
\Sigma \circ \Sigma=-\{\# \text { zeros of } \vec{v}\}=-\chi(\Sigma)
$$

by the Poincaré Hopf index theorem. Here the zeros of the vector field of course have to be counted with signs and multiplicities, i.e by their index. The overall sign in (3.7) is subtle but can easily be fixed by a local model. Note that it is not an independent choice of convention, but rather fixed by sign conventions for the index in vector fields and the convention for self-intersections.

Now $\bar{\sigma}$ acts freely on $\mathcal{M}-\Sigma$ and therefore

$$
\chi(\mathcal{M} / \bar{\sigma})=\frac{\chi(\mathcal{M})-\chi(\Sigma)}{2}+\chi(\Sigma)=12+\frac{\chi(\Sigma)}{2} .
$$


So the homology of $\mathcal{M} / \bar{\sigma}$ is (since $\bar{\sigma}$ preserves the top class):

$$
\operatorname{dim}_{\mathbb{R}}\left(H_{i}(\mathcal{M} / \bar{\sigma} ; \mathbb{R})\right)=\left\{\begin{array}{cc}
1 & i=4 \\
0 & i=3 \\
10+\frac{\chi(\Sigma)}{2} & i=2 \\
0 & i=1 \\
1 & i=0
\end{array}\right.
$$

and the dimension of the invariant subspace of $H_{2}(X ; \mathbb{R})$ has to be

$$
b_{2}^{+}=10+\frac{\chi(\Sigma)}{2} \Rightarrow b_{2}^{-}=22-b_{2}^{+}=12+\frac{\chi(\Sigma)}{2} .
$$

Combining (3.10) and (3.7) we find

$$
\Sigma \circ \Sigma=2\left(10-b_{2}^{+}\right) \quad \Leftrightarrow \quad \pi_{\mathrm{O} 7} \circ \pi_{\mathrm{O} 7}=2\left(9-n_{\mathrm{T}}\right) .
$$

\section{Examples on $\mathrm{K3}$}

We now present a number of examples of six-dimensional orientifolds on K3 with intersecting D7-branes and O7-planes. We start with a number of orbifold models, then discuss the quartic in $\mathbb{C P}^{3}$, and finally add a number of observations on the lift of supersymmetric models to F-theory on a Calabi-Yau 3-fold.

\subsection{Preliminaries on K3 orbifolds}

In the following we study certain orbifold realizations of $\mathrm{K} 3$ and provide evidence that the picture developed above applies. We consider the $\left\{\mathbb{Z}_{2}, \mathbb{Z}_{3}, \mathbb{Z}_{4}, \mathbb{Z}_{6}\right\}$ orbifold limits of $\mathrm{K} 3$, where the action of $\mathbb{Z}_{N}$ on the $z_{1}, z_{2}$ of (2.21) is defined by $v_{I}=(1 / N,-1 / N)$ as in (2.22). Recall, that the spectrum in table 1 is meant to be computed using the intersection numbers on the resolved orbifold and not on the initial torus. There are some 2-cycles $\pi_{a}$ on the $\mathrm{K} 3$ which are inherited from the torus. In the KK reduction on the orbifold they correspond to massless modes in the untwisted sector of the theory. In general 2-cycles $\bar{\pi}_{a}$ on the torus are arranged in orbits of length $N$ under the $\mathbb{Z}_{N}$ orbifold group, i.e.

$$
\pi_{a}=\sum_{i=0}^{N-1} \Theta^{i} \bar{\pi}_{a}
$$

Such an orbit can then be considered as a 2-cycle of the orbifold, where the intersection form is given by

$$
\pi_{a} \circ \pi_{b}=\frac{1}{N}\left(\sum_{i=0}^{N-1} \Theta^{i} \bar{\pi}_{a}\right) \circ\left(\sum_{j=0}^{N-1} \Theta^{j} \bar{\pi}_{b}\right) .
$$


Beside these 2-cycles the fixed points of the orbifold action give rise to exceptional 2-cycles, which correspond to massless fields in the twisted sectors of the orbifold. Blowing up the $i$-th $\mathbb{Z}_{N}$ singularity yields $(N-1)$ 2-cycles, $e_{i}^{(j)}, j \in\{1, \ldots, N-1\}$, whose intersection matrix is given by the Cartan matrix of the Lie algebra $A_{N-1}$, which is encoded in its Dynkin diagram.

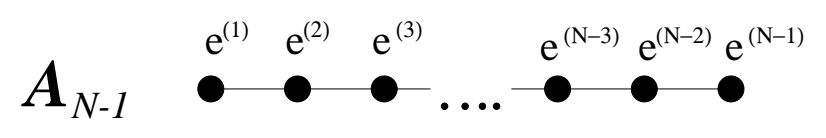

Figure 1

Note, that the cycles $\pi_{a}$ and $e_{i}^{(j)}$ in general do not yield an integral basis of $H_{2}(\mathrm{~K} 3, \mathbb{Z})$, but instead only generate a sub-lattice, while still of dimension 22, of course. Nevertheless, it provides the most convenient basis to work with when studying orientifolds, as the homology class of the orientifold plane and the action of the orientifold projection on these 2-cycles can easily be determined.

As was anticipated in [51], $\Omega \bar{\sigma}$ leaves all twisted sectors invariant and only leads to tadpoles from untwisted sectors. Thus, after blowing up the orbifold singularities, the O7-planes only wrap 2-cycles $\pi_{a}$ inherited from the torus and no exceptional divisors. To find $\pi_{\mathrm{O} 7}$ one then only needs to apply (2.25). By the relation (3.5), knowing only the orientifold plane is already sufficient to determine the contribution of the chiral spectrum of open strings to the gravitational anomaly. As an independent check, the action of $\Omega \bar{\sigma}$ on $H_{2}(\mathrm{~K} 3, \mathbb{Z})$ can then be used to compute $n_{\mathrm{T}}$ from $(2.20)$.

The action $[\bar{\sigma}]$ of $\bar{\sigma}$ on the cohomology of K3 was discussed in section 2.2 and can straightforwardly be determined. At the orbifold point all twisted sectors organize into singlets and doublets under $\Omega \bar{\sigma}$. Taking the intrinsic reflection into account as before, we write $[\bar{\sigma}]=(-1) \otimes p$, denoting the permutation of twisted sectors by $p$. In the smooth case, we then expect $[\bar{\sigma}]$ to act in an unchanged manner,

$$
e_{i}^{(j)} \mapsto-e_{p(i)}^{(j)} \quad \text { for } \quad j \in\{1, \ldots, N-1\}
$$

Before moving forward to study explicit blown-up $\mathbb{Z}_{N}$ orbifolds in more detail let us define a few useful conventions for the set of 2-cycles $\bar{\pi}_{a}$ on the torus. Within the framework of the four $\mathbb{Z}_{N}$ orbifolds of K3 which we are going to consider it turns out that each model allows two inequivalent complex conjugations $\bar{\sigma}$, depending on the complex structure of the background torus. For the case of $\left\{\mathbb{Z}_{2}, \mathbb{Z}_{4}\right\}$ we shall employ, up to rescaling, the root 
lattice of $S U(2)^{4}$, and for $\left\{\mathbb{Z}_{3}, \mathbb{Z}_{6}\right\}$ that of $S U(3)^{2}$. The complex structure $\tau^{I}$ of any single $T_{I}^{2}$ is defined by selecting two lattice vectors $\mathbf{e}_{1}^{I}=1, \mathbf{e}_{2}^{I}=\tau^{I}$ as a basis, where $\bar{\sigma}$ reflects along $\mathbf{e}_{1}^{I}$. As in [54] we define the choice $\mathbf{A}$ for the two lattices by taking

$$
\tau_{\mathbf{A}}=i, \quad \tau_{\mathbf{A}}=e^{2 \pi i / 6}
$$

for $S U(2)^{2}$ and $S U(3)$, respectively. The elementary cells of this type $\mathbf{A}$ are displayed in figure 2 .
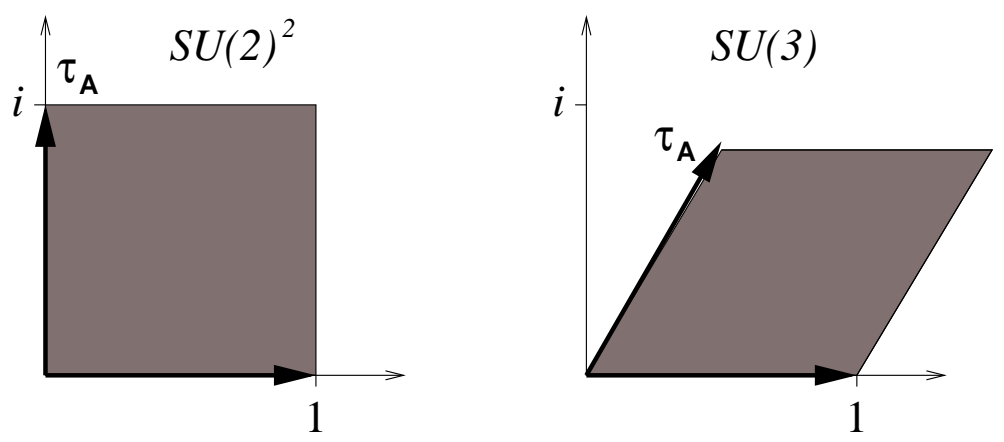

Figure 2

The case B B now refers to using the reflection symmetry of the lattices along the diagonal of these cells $\mathbf{A}$, defined by $\mathbf{e}_{1}+\mathbf{e}_{2}$. Therefore, the $\mathbf{B}$ type elementary cell is obtained by applying a rotation and a rescaling to the $\mathbf{A}$ type. The respective complex structures are given by

$$
\tau_{\mathbf{B}}=\frac{1+i}{2}, \quad \tau_{\mathbf{B}}=\frac{1}{2}+\frac{i}{2 \sqrt{3}}
$$

for $S U(2)^{2}$ and $S U(3)$. The $\mathbf{B}$ type elementary cells are depicted in figure 3 .
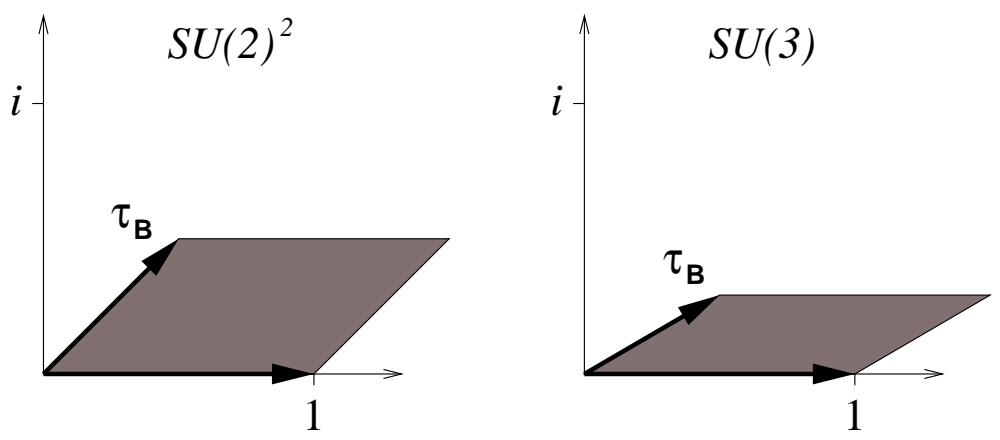

Figure 3 
Note, that in type I theory the real parts of the Kähler moduli are frozen and may take values $0,1 / 2$ only. In the present dual model, where $\Omega \bar{\sigma}$ is projected out, this requirement translates into demanding a crystallographic action of $\bar{\sigma}$ leading to $\Re\left(\tau^{I}\right)=0,1 / 2$.

For convenience, we now pick a fixed basis for $H_{2}\left(T^{4} ; \mathbb{Z}\right)$ and use it in both cases, which implies slightly more complicated formulas for the $\mathbf{B}$ type models. Given the $\mathbf{A}$ type lattice $\mathbf{e}_{1}^{I}=1$ and $\mathbf{e}_{2}^{I}=\tau_{\mathbf{A}}^{I}$, we define a basis $\left\{\gamma_{i}\right\}, i=1, \ldots, 4$, of $H_{1}\left(T^{4} ; \mathbb{Z}\right)$ by the four circles spanned by the basis vectors, $\gamma_{i+2(I-1)}=\left[\left\langle\mathbf{e}_{i}^{I}\right\rangle\right]$. Then

$$
\bar{\pi}_{i j}=\gamma_{i} \otimes \gamma_{j}
$$

is a basis for $H_{2}\left(T^{4} ; \mathbb{Z}\right)$. With this choice, the action of $[\bar{\sigma}]$ is very simple for the $\mathbf{A}$ type cells, $[\bar{\sigma}]$ leaves just the 1-cycles along $\mathbf{e}_{1}^{I}$ invariant, whereas for the $\mathbf{B}$ type, those along $\mathbf{e}_{1}^{I}+\mathbf{e}_{2}^{I}$ are invariant. By tensoring these together, one finds three inequivalent combinations $\{\mathbf{A A}, \mathbf{A B}, \mathbf{B B}\}$ for any orbifold group. The three models will also differ in the transformation of the fixed points of the orbifold group generator under $\bar{\sigma}$, which also depends on $\tau^{I}$.

\subsection{The orbifold limit $T^{4} / \mathbb{Z}_{2}$}

The simplest case to consider is the orbifold defined by $\Theta$ which reflects all four coordinates $\Theta: x_{i} \mapsto-x_{i}, i \in\{1,2,3,4\}$ of the $T^{4}$. The sixteen fixed points under the $\mathbb{Z}_{2}$ are given by $\left\{\left(0,1 / 2, \tau^{1} / 2,\left(1+\tau^{1}\right) / 2\right) \times\left(0,1 / 2, \tau^{2} / 2,\left(1+\tau^{2}\right) / 2\right)\right\}$. We denote them as $P_{i j}$ with $i, j \in\{1,2,3,4\}$. As defined by (4.6), there are six elements $\bar{\pi}_{i j}$ in $H_{2}\left(T^{4}, \mathbb{Z}\right)$, which we denote as

$$
\left\{\bar{\pi}_{13}, \bar{\pi}_{24}, \bar{\pi}_{14}, \bar{\pi}_{23}, \bar{\pi}_{12}, \bar{\pi}_{34}\right\}
$$

Their intersection form reads

$$
I_{T^{4}}=\left(\begin{array}{ll}
0 & 1 \\
1 & 0
\end{array}\right) \otimes \operatorname{diag}(1,-1,-1)
$$

Each of these six 2-cycles $\bar{\pi}_{i j}$ on $T^{4}$ gives rise to a 2 -cycle on the orbifold, $\pi_{i j}$, by taking its orbit under $\Theta$. As elements of $H_{2}\left(T^{4}, \mathbb{R}\right)$ they are simply invariant and thus also generators of $H_{2}\left(T^{4} / \mathbb{Z}_{2}, \mathbb{R}\right)$. But defining the image classes in $H_{2}\left(T^{4} / \mathbb{Z}_{2}, \mathbb{Z}\right)$ requires the proper normalization. It is evident, that a D7-brane wrapping along any of the elementary cycles is in general mapped to a different position on the torus by $\Theta$. The only exception occurs, if it is located within $\left.\left.\operatorname{Fix}(\bar{\sigma})\right|_{T^{4}} \cup \operatorname{Fix}(\Theta \bar{\sigma})\right|_{T^{4}}$. In this case, it could only be moved 
outside of this locus, if there were a second copy available, as its image under $\Theta$. Otherwise it is stuck to the fixed locus of $\bar{\sigma}$ and fractional in the physical sense of carrying only $1 / 2$ of the untwisted RR charge of a bulk brane. Thus, we conclude that the cycles descending from the cycles of the $T^{4}$ all appear as elements $\pi_{i j}=2 \bar{\pi}_{i j}$ in $H_{2}\left(T^{4} / \mathbb{Z}_{2}, \mathbb{Z}\right)$, corresponding to bulk D7-branes. Applying (4.2) yields their intersection matrix

$$
I_{T^{4} / \mathbb{Z}_{2}}^{\text {Torus }}=2 I_{T^{4}}
$$

on the orbifold. The remaining 16 2-cycles of the $\mathrm{K} 3$ are the blown-up $\mathbb{C P}^{1}$ at the 16 fixed points $P_{i j}$. Let us denote these sixteen exceptional divisors as $e_{i j}$ analogously. They have vanishing intersection with the six 2-cycles inherited from the $T^{4}$ and among themselves the intersections read

$$
e_{i j} \circ e_{k l}=-2 \delta_{i k} \delta_{j l}
$$

This is the Cartan matrix of $A_{1}^{16}$. Together this set of 2-cycles $\left\{\pi_{i j}, e_{i j}\right\}$ generates the socalled Kummer lattice, an index two sublattice of $H_{2}(\mathrm{~K} 3, \mathbb{Z}) \cong \Gamma_{3,19}$. In order to generate the entire lattice, we would also have to add fractional branes wrapping on cycles of the kind $\frac{1}{2} \pi_{13}+\frac{1}{2}\left(e_{11}+e_{12}+e_{21}+e_{22}\right)$, which is not part of the Kummer lattice. One always needs $N$ fractional branes to form a bulk D7-brane in a $\mathbb{Z}_{N}$ orbifold.

Now, we come to perform the orientifold by $\Omega \bar{\sigma}$ of the smooth K3 manifold. As was explained above, we can define anti-holomorphic involutions $\bar{\sigma}$ which act crystallographically on the $T^{4}$ with three different choices of the complex structure of $T_{I}^{2}$, labeled by $\{\mathbf{A A}, \mathbf{A B}, \mathbf{B B}\}$. 


\subsubsection{AA-orientifold}

Let us first choose $\tau_{\mathbf{A}}^{I}=i$ on both $T_{I}^{2}$, the AA model. The homology class of the O7-plane is

$$
\pi_{\mathrm{O} 7}=2\left(\pi_{13}+\pi_{24}\right) .
$$

With this choice of complex structure all the fixed points $P_{i j}$ are geometrically invariant. Through the intrinsic reflection of the blow-up mode, $[\bar{\sigma}]$ then reflects all $e_{i j}$. On $T^{4}$ only the cycles $\bar{\pi}_{13}$ and $\bar{\pi}_{24}$ are invariant. The action of $[\bar{\sigma}]$ on the 22 two-cycles is then given by

$$
[\bar{\sigma}]_{\mathbf{A A}}=\operatorname{diag}\left(\mathbf{1}_{2},-\mathbf{1}_{20}\right),
$$

with $\mathbf{1}_{n}$ denoting unit matrices of rank $n$. This also implies, that the image under $\Omega \bar{\sigma}$ of any brane with non-vanishing twisted charge always carries the opposite twisted charge. The number of tensor multiplets is now found to be $n_{\mathrm{T}}=1$. Computing the self-intersection number of the orientifold plane (4.11) we find $\pi_{\mathrm{O} 7} \circ \pi_{\mathrm{O} 7}=16$ so that indeed the $R^{4}$ anomaly is canceled. Now, we have all the ingredients required to compute the chiral spectrum of a general intersecting brane world on the $\mathrm{K} 3$ given by a blow-up of the orbifold $T^{4} / \mathbb{Z}_{2}$. It is easy to see that as long as we only introduce bulk branes the spectrum in table 1 agrees with the spectrum found in [7] for this case.

In fact, for the simple case of a $\mathbb{Z}_{2}$ orbifold group, the projection by $\Omega \bar{\sigma}$ is equivalent to the standard projection by $\Omega$ upon performing T-dualities along the two circles parameterized by $\Im\left(z_{i}\right)$. Let us therefore show as a check of our methods, that we can recover a T-dual version of the original $\mathbb{Z}_{2}$ orientifold first discovered by Bianchi and Sagnotti in [49 and described in terms of D-branes by Gimon and Polchinski 48]. The tadpoles can be canceled by introducing just two stacks of fractional D7-branes with multiplicities $N_{1}=N_{2}=16$, which support a gauge group $U(16)^{2}$. The cycles and their $\Omega \bar{\sigma}$ images they are wrapped around are

$$
\begin{aligned}
\pi_{1} & =\frac{1}{2}\left(\pi_{13}\right)+\frac{1}{2}\left(e_{11}+e_{12}+e_{21}+e_{22}\right), \\
\pi_{1}^{\prime} & =\frac{1}{2}\left(\pi_{13}\right)-\frac{1}{2}\left(e_{11}+e_{12}+e_{21}+e_{22}\right), \\
\pi_{2} & =\frac{1}{2}\left(\pi_{24}\right)+\frac{1}{2}\left(e_{11}+e_{13}+e_{31}+e_{33}\right), \\
\pi_{2}^{\prime} & =\frac{1}{2}\left(\pi_{24}\right)-\frac{1}{2}\left(e_{11}+e_{13}+e_{31}+e_{33}\right) .
\end{aligned}
$$


Their intersection numbers

$$
\begin{aligned}
& \pi_{1} \circ \pi_{1}=-2, \quad \pi_{2} \circ \pi_{2}=-2, \quad \pi_{1} \circ \pi_{1}^{\prime}=2, \quad \pi_{2} \circ \pi_{2}^{\prime}=2, \\
& \pi_{1} \circ \pi_{\mathrm{O} 7}=2, \quad \pi_{2} \circ \pi_{\mathrm{O} 7}=2, \quad \pi_{1} \circ \pi_{2}=0, \quad \pi_{1} \circ \pi_{2}^{\prime}=1
\end{aligned}
$$

yield the chiral massless spectrum shown in table 2

\begin{tabular}{|c|c|}
\hline Representation & Multiplicity \\
\hline$[(\mathbf{A d j}, \mathbf{1})+(\mathbf{1}, \mathbf{A d j})]_{(2,1)}$ & 2 \\
{$[(\mathbf{A}, \mathbf{1})+(\mathbf{1}, \mathbf{A})+\text { c.c. }]_{(1,2)}$} & 2 \\
{$[(\mathbf{1 6}, \mathbf{1 6})+\text { c.c. }]_{(1,2)}$} & 1 \\
\hline
\end{tabular}

Table 2: $\quad$ GP model

which agrees precisely with the massless spectrum determined in [49,48. Note, that in the orbifold limit the gauginos in the adjoint representation of the gauge group appeared in the sector of open strings with both ends on the same D7-brane. Chirality was induced by a non-trivial projection on the Chan-Paton indices, but the naive self-intersection of the brane was vanishing. However, in the smooth case the gauginos are localized at the self-intersections of the D7-branes. This self-intersection point was somehow hidden in the orbifold singularity and encoded in the action of the orbifold generator on the Chan-Paton indices. To make this whole picture consistent, it was absolutely crucial that the selfintersection number of an exceptional divisor is $e_{i j} \circ e_{i j}=-2$. At the orbifold point the model is supersymmetric, as here the D7-branes simply lie on top of the orientifold plane. Changing the Kähler structure by blowing-up the sixteen $\mathbb{Z}_{2}$ singularities does not break supersymmetry, as long as each individual D7-brane wraps a cycle calibrated by $\Re\left(\Omega_{2}\right)$.

Since the D-branes in (4.13) do wrap exceptional divisors, the tadpoles of the orientifold planes are not canceled locally in this model. To cancel them locally, one must introduce four stacks of $N=4$ D7-branes wrapped on the cycles

$$
\tilde{\pi}_{1}=\pi_{1}+\pi_{1}^{\prime}=\pi_{13}, \quad \tilde{\pi}_{2}=\pi_{2}+\pi_{2}^{\prime}=\pi_{24}
$$

Since these cycles are invariant under $\Omega \bar{\sigma}$ one gets an overall gauge group $S O(4)^{4} \times$ $S O(4)^{4}=S U(2)^{8} \times S U(2)^{8}$. Note, that this should be precisely the model for which Ashoke Sen presented an F-theory dual description in [70,71]. We will discuss the implications of this dual F-theory picture in section 4.7.1. 
Let us consider another example with fractional D-branes that had appeared in [7]. The tadpoles can also be canceled by just a single stack of $N=16$ branes, together with its image under $\Omega \bar{\sigma}$. It is wrapped on

$$
\begin{aligned}
\pi & =\frac{1}{2}\left(\pi_{13}+\pi_{24}+\pi_{14}+\pi_{23}\right)+\frac{1}{2}\left(e_{11}+e_{44}+e_{14}+e_{41}\right), \\
\pi^{\prime} & =\frac{1}{2}\left(\pi_{13}+\pi_{24}-\pi_{14}-\pi_{23}\right)-\frac{1}{2}\left(e_{11}+e_{44}+e_{14}+e_{41}\right) .
\end{aligned}
$$

Thus, we get a gauge group $U(16)$. For the relevant intersection numbers we obtain

$$
\pi \circ \pi=-2, \quad \pi \circ \pi^{\prime}=4, \quad \pi \circ \pi_{\mathrm{O} 7}=4,
$$

giving rise to the chiral massless spectrum

\begin{tabular}{|c|c|}
\hline Representation & Multiplicity \\
\hline$[\mathbf{A d j}]_{(2,1)}$ & 2 \\
{$[\mathbf{A}+\overline{\mathbf{A}}]_{(1,2)}$} & 4 \\
\hline
\end{tabular}

Table 3: Chiral spectrum

This is precisely the spectrum obtained in the orbifold limit [7]. Computing the disc level scalar potential (2.17) at the orbifold point one finds

$$
\mathcal{V}=T_{7} e^{-\phi_{6}}\left[\prod_{I=1}^{2} \sqrt{\Im\left(\tau^{I}\right)+\frac{1}{\Im\left(\tau^{I}\right)}}-\left(\sqrt{\Im\left(\tau^{1}\right) \Im\left(\tau^{2}\right)}+\frac{1}{\sqrt{\Im\left(\tau^{1}\right) \Im\left(\tau^{2}\right)}}\right)\right]
$$

which vanishes precisely if the two complex structure moduli $\Im\left(\tau^{1}\right)$ and $\Im\left(\tau^{2}\right)$ for the two $T_{I}^{2}$ are equal. This is the point, where the angle criterion (2.29) for the D7-branes is satisfied and the configuration turns supersymmetric. Moving away from the supersymmetric $\Im\left(\tau^{1}\right)=\Im\left(\tau^{2}\right)$ locus, the intersection angles do not any longer satisfy (2.29), supersymmetry is broken and an open string tachyon appears. In the effective six-dimensional gauge theory this effect is described by a Fayet-Iliopoulos term depending on $\xi \sim \Im\left(\tau^{1}\right)-\Im\left(\tau^{2}\right)$ [72]. Note, that in six dimensions the D-term potential for a $U(1)$ gauge field has the general form

$$
\mathcal{V}_{\text {D-term }} \sim\left(\sum_{i} q^{i}\left|\phi_{i}\right|^{2}-\sum_{i} q^{i}\left|\widetilde{\phi}_{i}\right|^{2}-\xi\right)^{2},
$$


where $\phi_{i}$ and $\widetilde{\phi}_{i}$ denote the two complex scalars insider a hypermultiplet $H_{i}$ with charge $q_{i}$. Note that independent of the sign of $\xi$, if $\xi \neq 0$ one always gets a tachyonic mode, which is in accord with the string theory picture.

Let us conclude this example with stating that here supersymmetry and tadpole cancellation conditions still allow non-trivial D7-brane intersections with chiral matter.

\subsubsection{AB-orientifold}

In the case of an $\mathbf{A B}$ complex structure, the O7-plane wraps around the homological cycle

$$
\pi_{\mathrm{O} 7}=\pi_{13}+\pi_{24}+\pi_{14}-\pi_{23} .
$$

On the exceptional divisors $\left\{e_{11}, e_{21}, e_{31}, e_{41}, e_{14}, e_{24}, e_{34}, e_{44}\right\} \bar{\sigma}$ acts with a minus sign, and the four cycles $\left\{e_{12}, e_{22}, e_{32}, e_{42}\right\}$ get exchanged with $\left\{e_{13}, e_{23}, e_{33}, e_{43}\right\}$. Its action on $\mathrm{H}_{2}(\mathrm{~K} 3, \mathbb{Z})$ is summarized by

$$
[\bar{\sigma}]_{\mathrm{AB}}=\left(\left(\begin{array}{ll}
0 & 1 \\
1 & 0
\end{array}\right) \otimes \operatorname{diag}(1,-1)\right) \oplus\left(-\mathbf{1}_{2}\right) \oplus \bigoplus_{k=1}^{4}\left(\begin{array}{cc}
0 & -1 \\
-1 & 0
\end{array}\right)_{k} \oplus\left(-\mathbf{1}_{8}\right) .
$$

The first two terms stand for the cycles inherited from the torus, the last two for the action on the exceptional cycles. Hence, the number of tensor-multiplets is $n_{\mathrm{T}}=5$, one from the torus and four from the blow-up modes, which is in accord with the self-intersection number of the orientifold plane, $\pi_{\mathrm{O} 7} \circ \pi_{\mathrm{O} 7}=8$.

\subsubsection{BB-orientifold}

If one chooses the $\mathbf{B}$ type complex structure on both $T_{I}^{2}$ the orientifold plane is wrapping

$$
\pi_{\mathrm{O} 7}=\pi_{13}+\pi_{24}
$$

with self-intersection number $\pi_{\mathrm{O} 7} \circ \pi_{\mathrm{O} 7}=4$. On the 2-cycles $\bar{\sigma}$ acts like

$$
[\bar{\sigma}]_{\mathrm{BB}}=\left(\begin{array}{ll}
0 & 1 \\
1 & 0
\end{array}\right) \oplus\left(\begin{array}{cc}
0 & -1 \\
-1 & 0
\end{array}\right) \oplus\left(-\mathbf{1}_{2}\right) \oplus \bigoplus_{k=1}^{6}\left(\begin{array}{cc}
0 & -1 \\
-1 & 0
\end{array}\right)_{k} \oplus\left(-\mathbf{1}_{4}\right) .
$$

This time, $\bar{\sigma}$ acts on the four exceptional divisors $\left\{e_{11}, e_{14}, e_{41}, e_{44}\right\}$ with -1 , and the six exceptional 2-cycles $\left\{e_{12}, e_{42}, e_{21}, e_{24}, e_{22}, e_{23}\right\}$ get exchanged with $\left\{e_{13}, e_{43}, e_{31}, e_{34}, e_{33}, e_{32}\right\}$. We get $n_{\mathrm{T}}=7$ tensor multiplets, again one from the torus plus six from the blow-up modes. 
As an example for a solution to the tadpole constraints, we introduce two stacks of fractional D7-branes with $N_{1}=N_{2}=8$, which support a gauge group $U(8)^{2}$. They wrap the following 2-cycles

$$
\begin{aligned}
\pi_{1} & =\frac{1}{2}\left(\pi_{13}\right)+\frac{1}{2}\left(e_{11}+e_{12}+e_{21}+e_{22}\right), \\
\pi_{1}^{\prime} & =\frac{1}{2}\left(\pi_{24}\right)+\frac{1}{2}\left(-e_{11}+e_{13}+e_{31}+e_{33}\right), \\
\pi_{2} & =\frac{1}{2}\left(\pi_{13}\right)-\frac{1}{2}\left(e_{11}+e_{12}+e_{21}+e_{22}\right), \\
\pi_{2}^{\prime} & =\frac{1}{2}\left(\pi_{24}\right)-\frac{1}{2}\left(-e_{11}+e_{13}+e_{31}+e_{33}\right) .
\end{aligned}
$$

The relevant intersection numbers

$$
\begin{aligned}
& \pi_{1} \circ \pi_{1}=-2, \quad \pi_{2} \circ \pi_{2}=-2, \quad \pi_{1} \circ \pi_{1}^{\prime}=1, \quad \pi_{2} \circ \pi_{2}^{\prime}=1, \\
& \pi_{1} \circ \pi_{\mathrm{O} 7}=1, \quad \pi_{2} \circ \pi_{\mathrm{O} 7}=1, \quad \pi_{1} \circ \pi_{2}=2, \quad \pi_{1} \circ \pi_{2}^{\prime}=0
\end{aligned}
$$

yield the chiral massless spectrum

\begin{tabular}{|c|c|}
\hline Representation & Multiplicity \\
\hline$[(\mathbf{A d j} \mathbf{j}, \mathbf{1})+(\mathbf{1}, \mathbf{A d j})]_{(2,1)}$ & 2 \\
{$[(\mathbf{A}, \mathbf{1})+(\mathbf{1}, \mathbf{A})+\text { c.c. }]_{(1,2)}$} & 1 \\
{$[(\mathbf{8}, \mathbf{8})+\text { c.c. }]_{(1,2)}$} & 2 \\
\hline
\end{tabular}

Table 4: Chiral spectrum

This agrees completely with the spectrum derived in the orbifold limit [7].

\subsection{The orbifold limit $T^{4} / \mathbb{Z}_{3}$}

For the orbifold of $T^{4} / \mathbb{Z}_{3}$ we in principle again have to distinguish three inequivalent choices of the complex structure of the $T^{4}$, which is now defined by the root lattice of $S U(3)^{2}$. In any case only four 2-cycles are inherited from the torus, which can be described as orbits under the action of the $\mathbb{Z}_{3}$ by

$$
\begin{aligned}
& \pi_{1}=\bar{\pi}_{13}+\bar{\pi}_{14}+\bar{\pi}_{23}-2 \bar{\pi}_{24}, \\
& \pi_{2}=2 \bar{\pi}_{14}+2 \bar{\pi}_{23}-\bar{\pi}_{13}-\bar{\pi}_{24}, \\
& \pi_{3}=3 \bar{\pi}_{12}, \\
& \pi_{4}=3 \bar{\pi}_{34} .
\end{aligned}
$$


The intersection matrix for these four 2-cycles can be computed using (4.2) to be

$$
I_{T^{4} / \mathbb{Z}_{3}}^{\text {Torus }}=\left(\begin{array}{ll}
2 & 1 \\
1 & 2
\end{array}\right) \oplus\left(\begin{array}{ll}
0 & 3 \\
3 & 0
\end{array}\right) .
$$

The remaining 18 2-cycles result from blowing-up the $9 A_{2}$ singularities. For each fixed point we get two 2-cycles $e_{i j}^{(1)}, e_{i j}^{(2)}$ with their intersection matrix given by the Cartan matrix of $A_{2}$. The total intersection matrix is given by

$$
I_{T^{4} / \mathbb{Z}_{3}}=\bigoplus_{k=1}^{9}\left(\begin{array}{cc}
-2 & 1 \\
1 & -2
\end{array}\right)_{k} \oplus I_{T^{4} / \mathbb{Z}_{3}}^{\text {Torus }}
$$

In the following, we shall be very brief on the model defined by the involution $\Omega \bar{\sigma}$ applied to type IIB on $T^{4} / \mathbb{Z}_{3}$, but add an excursion on a related class of models.

\subsubsection{Anti-holomorphic involution $\bar{\sigma}$}

For the orbifold $T^{4} / \mathbb{Z}_{3}$ there again exist two possible choices for the complex structure of each $T_{I}^{2}$ leading to three different models. The computation of the homological cycle of the O7-plane and the action of $\Omega \bar{\sigma}$ on the basis of $H_{2}(\mathrm{~K} 3, \mathbb{Z})$ can be determined straightforwardly. We summarize the main data in table 5.

\begin{tabular}{|c|c|c|c|}
\hline Complex structure & $\pi_{\mathrm{O} 7}$ & $\pi_{\mathrm{O} 7} \circ \pi_{\mathrm{O} 7}$ & $n_{\mathrm{T}}$ \\
\hline $\mathbf{A A}$ & $\pi_{1}$ & 2 & 8 \\
\hline $\mathbf{A B}$ & $\pi_{1}+\pi_{2}$ & 6 & 6 \\
\hline $\mathbf{B B}$ & $3 \pi_{2}$ & 18 & 0 \\
\hline
\end{tabular}

Table 5: $\quad T^{4} / \mathbb{Z}_{3}$ orientifolds

Using these data one can check that placing the D7-branes on top of the orientifold planes leads precisely to the chiral massless spectra obtained in [51,53] in the orbifold limit. More general models can easily be constructed in great numbers.

\subsubsection{Holomorphic involution $\sigma$}

On a K3 surface the notion of a special Lagrangian cycle and a complex curve, which refer to a particular choice of the Kähler and the holomorphic 2-form, can get exchanged. 
This is done by a rotation of the complex structure, which is discussed in some more detail in section 4.6. This rotation maps the complex conjugation $\bar{\sigma}$ to

$$
\sigma:\left(z_{1}, z_{2}\right) \mapsto\left(z_{1},-z_{2}\right)
$$

which now reflects the holomorphic 2 -form, not just its imaginary part. In contrast to the earlier case, we now have that

$$
\left.\Omega_{2}\right|_{\operatorname{Fix}(\sigma)}=0,\left.\quad J\right|_{\operatorname{Fix}(\sigma)}=\left.d \operatorname{vol}\right|_{\operatorname{Fix}(\sigma)},
$$

i.e. $\operatorname{Fix}(\sigma)$ is a holomorphic cycle calibrated with respect to $J$. If we were just considering toroidal compactifications, the two descriptions would be strictly equivalent. But the definition of the orbifolds via (2.22) itself also implies a choice of a particular complex structure. In order to compensate for the action on the zero modes of the RR ground state, it is required to include an additional sign $(-1)^{F_{L}}, F_{L}$ the left-moving world sheet fermion number operator, with the world sheet parity $\Omega$. Then, combining projections by $\Omega \sigma(-1)^{F_{L}}$ and $\Theta$ leads to inequivalent results, as compared to $\Omega \bar{\sigma}$ and $\Theta$. In fact, the group generated by $\left\{\Omega \sigma(-1)^{F_{L}}, \Theta\right\}$ is the precise T-dual of $\{\Omega, \Theta\}$, after T-dualities along the two circles parameterized by $z_{2}$. On the contrary, the T-dualities along the imaginary parts of $z_{1}, z_{2}$ take $\{\Omega \bar{\sigma}, \Theta\}$ to $\{\Omega, \hat{\Theta}\}$, where $\hat{\Theta}$ is an asymmetric operation that treats left- and right-moving world-sheet fields with opposite phase factors [73]. Due to these relations, we can expect, that the models defined by $\sigma$ reproduce the known orientifold vacua of type I string theory [50]. To demonstrate this fact, we include the case of the $T^{4} / \mathbb{Z}_{3}$ orientifold with $\Omega \sigma$ projection here. For the even simpler case of $T^{4} / \mathbb{Z}_{2}$, the two types of models are still completely equivalent and the definition of $\Theta$ does not distinguish $\sigma$ and $\bar{\sigma}$.

An important ingredient in the CFT construction of orbifold vacua of type $\mathrm{I}$ is the action of $\Omega$ on twisted sectors. A field twisted by $\Theta^{k}$ is mapped to one twisted by $\Theta^{N-k}$ via $\Omega$. In contrast to the $\Omega \bar{\sigma}$ models, one then also gets twisted sector tadpoles. This means, that, after blowing up the orbifold singularities, the orientifold planes also wrap exceptional divisors. We propose that the action on the exceptional 2-cycles is then given by

$$
[\sigma]: e_{i}^{(j)} \mapsto-e_{i}^{(N-j)} \quad \text { for } j \in\{1, \ldots, N-1\}
$$




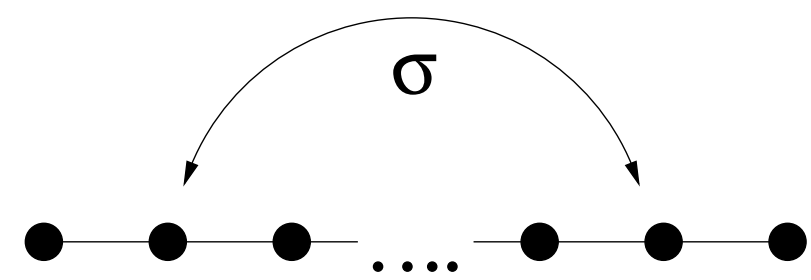

Figure 4

Thus, $\Omega \sigma$ reflects the Dynkin diagram, as depicted in figure 4 .

In other words, $\Omega \sigma$ exchanges the two blow-up modes $e_{i j}^{(1)}, e_{i j}^{(2)}$ of the two $\mathbb{C P}^{1}$ which are glued into any of the nine $A_{2}$ singularities at the fixed points of the $\mathbb{Z}_{3}$. Taking also the action of $\Omega \sigma$ on the four toroidal 2-cycles into account, which leaves one tensor multiplet, we deduce that we get $n_{\mathrm{T}}=10$ tensor multiplets in the closed string sector. At the four fixed points of $\sigma$ we get an O7-plane, which under the action of $\mathbb{Z}_{3}$ gives rise to one bulk O7-plane and one fractional O7-plane. The total homological cycle is given by

$$
\pi_{\mathrm{O} 7}=\pi_{12}+\left(\frac{1}{3} \pi_{12}+\frac{1}{\sqrt{3}}\left[e_{11}^{(1)}+e_{11}^{(2)}+e_{21}^{(1)}+e_{21}^{(2)}+e_{31}^{(1)}+e_{31}^{(2)}\right]\right)
$$

Note, that the fractional O7-plane is indeed invariant under $\Omega \sigma$. The self-intersection number of this O7-plane can be computed to be $\pi_{\mathrm{O} 7} \circ \pi_{\mathrm{O} 7}=-2$, which is consistent with having $n_{\mathrm{T}}=10$. One can cancel the RR-tadpoles by introducing 8 bulk D7-branes and 8 fractional D7-branes. Splitting the bulk branes into 3 fractional branes we get three kinds of D7-branes

$$
\begin{aligned}
N_{1}=16: & \pi_{1}=\frac{1}{3} \pi_{12}+\frac{1}{\sqrt{3}}\left[e_{11}^{(1)}+e_{11}^{(2)}+e_{21}^{(1)}+e_{21}^{(2)}+e_{31}^{(1)}+e_{31}^{(2)}\right], \\
N_{2}=8: & \pi_{2}=\frac{1}{3} \pi_{12}-\frac{1}{\sqrt{3}}\left[e_{11}^{(1)}+e_{21}^{(1)}+e_{31}^{(1)}\right], \\
N_{2}=8: & \pi_{2}^{\prime}=\frac{1}{3} \pi_{12}-\frac{1}{\sqrt{3}}\left[e_{11}^{(2)}+e_{21}^{(2)}+e_{31}^{(2)}\right] .
\end{aligned}
$$

Here, $\pi_{1}$ is $\Omega \sigma$-invariant, whereas $\pi_{2}$ and $\pi_{2}^{\prime}$ are mapped upon each other. The gauge group on an invariant fractional brane is given by $S O(N)$, while a fractional brane that belongs to an orbit of length 3 still carries a $U(N)$ gauge group. Together, the gauge group is $S O(16) \times U(8)$. From the intersection numbers

$$
\pi_{i} \circ \pi_{i}=-2, \quad \pi_{2} \circ \pi_{\mathrm{O} 7}=1, \quad \pi_{2} \circ \pi_{2}^{\prime}=1, \quad \pi_{1} \circ \pi_{2}=1
$$

we deduce the chiral massless spectrum by using table 1 , 


\begin{tabular}{|c|c|}
\hline Representation & Multiplicity \\
\hline$[(\mathbf{A d j}, \mathbf{1})+(\mathbf{1}, \mathbf{A d j})]_{(2,1)}$ & 2 \\
{$[(\mathbf{1}, \mathbf{A})+\text { c.c. }]_{(1,2)}$} & 1 \\
{$[(\mathbf{1 6}, \mathbf{8})+\text { c.c. }]_{(1,2)}$} & 1 \\
\hline
\end{tabular}

Table 6: $\quad \mathbb{Z}_{3}$ orientifolds

which agrees completely with the massless spectrum derived in [50] for the $\mathbb{Z}_{3}$ orientifold of type I string theory. We consider this as a very neat confirmation that the formula for the chiral massless spectrum is absolutely general and is expected to cover all six-dimensional orientifolds computed in the literature.

\subsection{The orbifold limit $T^{4} / \mathbb{Z}_{4}$}

For the orientifold $T^{4} / \mathbb{Z}_{4}$ we can again use the lattice defined by $S U(2)^{4}$. The resulting K3 manifold inherits the following four 2-cycles from the torus

$$
\begin{aligned}
& \pi_{1}=2 \bar{\pi}_{13}-2 \bar{\pi}_{24}, \\
& \pi_{2}=2 \bar{\pi}_{14}+2 \bar{\pi}_{23}, \\
& \pi_{3}=4 \bar{\pi}_{12}, \\
& \pi_{4}=4 \bar{\pi}_{34},
\end{aligned}
$$

which have the intersection matrix

$$
I_{T^{4} / \mathbb{Z}_{4}}^{\text {Torus }}=\left(\begin{array}{ll}
2 & 0 \\
0 & 2
\end{array}\right) \oplus\left(\begin{array}{ll}
0 & 4 \\
4 & 0
\end{array}\right) .
$$

Moreover, one has four $A_{3}$ singularities $\left\{e_{11}, e_{14}, e_{41}, e_{44}\right\}$ and six $A_{1}$ singularities, so that the complete intersection matrix is

$$
I_{T^{4} / \mathbb{Z}_{4}}=\bigoplus_{k=1}^{4}\left(\begin{array}{ccc}
-2 & 1 & 0 \\
1 & -2 & 1 \\
0 & 1 & -2
\end{array}\right) \bigoplus_{k} \oplus \bigoplus_{l=1}^{6}(-2)_{l} \oplus I_{T^{4} / \mathbb{Z}_{4}}^{\text {Torus }}
$$

In principle, we would have to distinguish the three choices of complex structure for the $T^{4}$ as before. But one can observe that the two models AA and $\mathbf{B B}$ are equivalent, as they give rise to the same orientifold plane. We summarize the main data for the two inequivalent orientifolds in table 7 . 


\begin{tabular}{|c|c|c|c|}
\hline Complex structure & $\pi_{\mathrm{O} 7}$ & $\pi_{\mathrm{O} 7} \circ \pi_{\mathrm{O} 7}$ & $n_{\mathrm{T}}$ \\
\hline $\mathbf{A A}$ or $\mathbf{B B}$ & $3 \pi_{1}$ & 18 & 0 \\
\hline $\mathbf{A B}$ & $2\left(\pi_{1}+\pi_{2}\right)$ & 16 & 1 \\
\hline
\end{tabular}

Table 7: $\quad T^{4} / \mathbb{Z}_{4}$ orientifolds

The result for the $\mathbf{A B}$ model agrees with the result obtained by an explicit CFT computation in [51]. However, for the AA and $\mathbf{B B}$ model it was argued in [51] that the corresponding cross-cap state was not well defined ${ }^{2}$.

\subsection{The orbifold limit $T^{4} / \mathbb{Z}_{6}$}

The torus defined by the lattice of $S U(3)^{2}$ does allow the action of a $\mathbb{Z}_{6}$ symmetry group. The orbifold inherits the following four 2-cycles from the $T^{4}$

$$
\begin{aligned}
& \pi_{1}=2 \bar{\pi}_{13}+2 \bar{\pi}_{23}+2 \bar{\pi}_{14}-4 \bar{\pi}_{24}, \\
& \pi_{2}=4 \bar{\pi}_{14}+4 \bar{\pi}_{23}-2 \bar{\pi}_{13}-2 \bar{\pi}_{24}, \\
& \pi_{3}=6 \bar{\pi}_{12}, \\
& \pi_{4}=6 \bar{\pi}_{34} .
\end{aligned}
$$

They have an intersection matrix

$$
I_{T^{4} / \mathbb{Z} 6}^{\text {Torus }}=\left(\begin{array}{ll}
4 & 2 \\
2 & 4
\end{array}\right) \oplus\left(\begin{array}{ll}
0 & 6 \\
6 & 0
\end{array}\right)
$$

Further, $\Theta$ has just one fixed point, an $A_{5}$ singularity $\left\{e_{11}\right\}, \Theta^{2}$ has four fixed points, $A_{2}$ singularities, and $\Theta^{3}$ five $A_{1}$ singularities. Together, the intersection matrix reads

$$
I_{T^{4} / \mathbb{Z}_{6}}=\left(\begin{array}{ccccc}
-2 & 1 & 0 & 0 & 0 \\
1 & -2 & 1 & 0 & 0 \\
0 & 1 & -2 & 1 & 0 \\
0 & 0 & 1 & -2 & 1 \\
0 & 0 & 0 & 1 & -2
\end{array}\right) \oplus \bigoplus_{k=1}^{4}\left(\begin{array}{cc}
-2 & 1 \\
1 & -2
\end{array}\right) \oplus \bigoplus_{k}^{5}(-2)_{l} \oplus I_{T^{4} / \mathbb{Z}_{6}}^{\text {Torus }}
$$

2 That argument stated that the relative normalization of the cross-cap states $\left|\Omega \bar{\sigma} \Theta^{k}\right\rangle$ must be fixed in such a way that in the overall tree-channel Klein-bottle amplitude only states invariant under $\Theta$ propagate. In view of the results in this section, we think this condition was a too strong requirement. Note, that the closed string exchange between two individual orientifold planes is simply given by the overlap $\left\langle\Omega \bar{\sigma} \Theta^{k}\left|e^{-l H_{\mathrm{cl}}}\right| \Omega \bar{\sigma} \Theta^{l}\right\rangle$, so that imposing conditions on the overall tree-channel Klein-bottle amplitude has no physical meaning. 
Again, the two models $\mathbf{A A}$ and $\mathbf{B B}$ are equivalent. We then summarize the data for the two inequivalent orientifolds in table 8 .

\begin{tabular}{|c|c|c|c|}
\hline Complex structure & $\pi_{\mathrm{O} 7}$ & $\pi_{\mathrm{O} 7} \circ \pi_{\mathrm{O} 7}$ & $n_{\mathrm{T}}$ \\
\hline $\mathbf{A A}$ or $\mathbf{B B}$ & $2 \pi_{1}$ & 16 & 1 \\
\hline $\mathbf{A B}$ & $\pi_{1}+\pi_{2}$ & 12 & 3 \\
\hline
\end{tabular}

Table 8: $\quad T^{4} / \mathbb{Z}_{6}$ orientifolds

\subsection{The quartic}

In this section we examine the most simple example of a $\mathrm{K} 3$ surface defined as a hypersurface in a projective space, the quartic polynomial in $\mathbb{C P}^{3}$. While we only discuss three special cases, these still turn out to give us rather exotic models very different from the orbifolds above.

The quartic is defined by an arbitrary polynomial in the four homogeneous coordinates $\left[z_{0}: z_{1}: z_{2}: z_{3}\right]$ that parameterize $\mathbb{C P}^{3}$, i.e.

$$
\sum_{i, j, k, l=0}^{3} a_{i j k l} z_{i} z_{j} z_{k} z_{l}=0
$$

The $35=4 \cdot 5 \cdot 6 \cdot 7 /(2 \cdot 3 \cdot 4)$ parameters $a_{i j k l}$ modulo the $16=4^{2}$ parameters of $G L(4, \mathbb{C})$ parameterize a nineteen-dimensional family of algebraic K3 surfaces. As long as all coefficients $a_{i j k l}$ are chosen real, the conjugation on the four $z_{i}$ is a symmetry, i.e. projecting by $\Omega \bar{\sigma}$ removes one half the moduli. The most simple case arises when there are no real solutions to (4.41). This is precisely the case when only quartic or quadratic terms have non-vanishing and positive coefficients

$$
\sum_{i=0}^{3}\left(a_{i} z_{i}^{4}+z_{i}^{2} \sum_{j<i} b_{i j} z_{j}^{2}\right)=0, \quad a_{i}, b_{i j} \geq 0,
$$

which, for $b_{i j}=0$, includes the Fermat quartic. Here $\operatorname{Fix}(\bar{\sigma})$ is empty and thus $\pi_{\mathrm{O} 7}=0$, so that $n_{\mathrm{T}}=9$ tensor multiplets are required by the anomaly cancellation. Therefore among the 22 two-cycles we expect 10 cycles to be invariant under $\bar{\sigma}$ and 12 cycles to be anti-invariant. Such a model was inaccessible in orbifold vacua, where the orbit of the 
real section on the torus always descends to an invariant locus under $\bar{\sigma}$. In fact there is a natural candidate for an orbifold model of type I strings which can produce models without orientifold planes. In order to achieve this, one combines the operation $\Omega$ with a freely acting shift. Its insertion in the Klein bottle does not produce massless tadpoles which would signal background charges of orientifold planes. In 66, 67 such an orientifold model was constructed, inspired by the existence of an F-theory compactification [74 believed to be dual to a type I vacuum without orientifold planes.

A slight modification of the above case leads to another model given by flipping the sign of any one of the $a_{i}$ coefficients, say $a_{0}=-1$. For simplicity, we also set $b_{i j}=0$ and scale $a_{i}=1, i>0$ in (4.42). By going to complex $a_{0}$ in between, this deformation is continuous, but passing through a region in the moduli space where $\bar{\sigma}$ is no symmetry anymore. Defining $x_{i}=\Re\left(z_{i}\right)$, the hypersurface intersects the real section in a sphere, parameterized by

$$
-x_{0}^{4}+x_{1}^{4}+x_{2}^{4}+x_{3}^{4}=0, \quad\left[x_{0}: x_{1}: x_{2}: x_{3}\right] \in \mathbb{R P}^{3} .
$$

For $x_{0}=0$ there is no solution, so we can rescale the homogeneous coordinates to $x_{0}=1$ :

$$
x_{1}^{4}+x_{2}^{4}+x_{3}^{4}=1, \quad\left(x_{1}, x_{2}, x_{3}\right) \in \mathbb{R}^{3} .
$$

Topologically $\operatorname{Fix}(\bar{\sigma})$ is thus given by a single calibrated 2-sphere. By the hyperkähler property of $\mathrm{K} 3$, this is a holomorphic $\mathbb{C P}^{1}$ in another complex structure, which is determined to have self-intersection $\pi_{\mathrm{O} 7} \circ \pi_{\mathrm{O} 7}=-2$. This would require $n_{\mathrm{T}}=10$ in accord with $\chi\left(\mathbb{C P}^{1}\right)=2$. Thus in this model the O7-plane is wrapping a single exceptional 2-cycle, which looks rather strange from the point of view of the orbifold models studied earlier. The 22 two-cycles must arrange themselves into $11 \bar{\sigma}$ invariant ones and the same number of anti-invariant ones.

Finally, consider the following quartic

$$
-z_{1}^{4}-z_{2}^{4}+z_{3}^{4}+z_{4}^{4}=0
$$

Here the real subspace $\operatorname{Fix}(\bar{\sigma})$ is a special Lagrangian two-torus, as was remarked in [75]. As such it has vanishing self-intersection. So there are $n_{\mathrm{T}}=9$ tensor multiplets as in the first case with empty $\operatorname{Fix}(\bar{\sigma})$. But of course now there are orientifold planes and D-branes, making the physics much more interesting. 


\subsection{Lift to F-theory on Calabi-Yau 3-folds}

In this section we will analyze the perspectives to lift the type IIB compactifications on K3 modded out by $\Omega \bar{\sigma}$ to F-theory [76, 77, 74 . In particular we want to make contact to earlier works on the analogous lift of the $T^{4} / \mathbb{Z}_{2}$ orientifold to an F-theory compactification on an elliptically fibered Calabi-Yau 3-fold. In certain prominent cases it can be of the Voisin-Borcea type. In general, the lift implies a geometric interpretation of the tendimensional complexified string coupling $\lambda$ as the complex structure of a complex torus fibered over the compactification space of type IIB. The degeneration locus of the fiber is then given by the locations of D7-branes and O7-planes. This implies that they wrap on complex cycles of the K3, cycles calibrated with respect to the Kähler structure $J$. As long as the RR charges carried by these objects are confined to very small regions on the internal space and at least approximately cancel out locally, the string coupling may be assumed to be constant and small outside this region. Therefore, the perturbative analysis in the CFT orbifold may be compared to the geometric picture. This local charge cancellation can no longer be achieved in general when the D7-branes and O7-planes wrap on different cycles of the K3.

\subsubsection{The Weierstraß model on $\mathbb{C P}^{1} \times \mathbb{C P}^{1}$}

In [71] Ashoke Sen gave a description of a Weierstraß model of the elliptic fibration

on the base space $\mathbb{C P}^{1} \times \mathbb{C P}^{1}$, which makes up a Calabi-Yau 3-fold with Hodge numbers $\left(h^{(1,1)}, h^{(2,1)}\right)=(3,243)$. The model is believed to be dual to the standard orientifold compactification of type IIB on $T^{4} / \mathbb{Z}_{2}$ with local charge cancellation. It was first considered in [49] and subsequently discussed in [48], and was subject of section 4.1. In the original formulation the group generator $\Theta$ reflects all four coordinates of the K3, $\Theta: \hat{x}_{i} \mapsto-\hat{x}_{i}, i=1, \ldots, 4$, and the orientifold group is generated by $\Omega$ and $\Theta$. The tadpole cancellation conditions imply the presence of 32 D9- and 32 D5-branes to cancel the background charges of the O9-planes and the O5-planes localized at the 16 fixed points of $\Theta$. The maximal gauge group is given by $U(16) \times U(16)$. This formulation is not quite adapted to an interpretation within the framework of F-theory, and one first needs to apply T-dualities along two of the circles of the $T^{4}$, say along $\hat{x}_{1}$ and $\hat{x}_{3}$. This maps

$$
\Omega \mapsto \Omega \Theta_{13}(-1)^{F_{L}}, \quad \Theta \mapsto \Theta,
$$


where $\Theta_{i j}$ now only reflects $\hat{x}_{i}$ and $\hat{x}_{j}$ and $F_{L}$ is the left-moving world sheet fermion number operator. The resulting group is generated by $\Omega \Theta_{13}(-1)^{F_{L}}$ and $\Omega_{24}(-1)^{F_{L}}$. The former D9- and D5-branes, as well as the O-planes, map to intersecting D7-branes and O7-planes located in the fixed loci of $\Theta_{13}$ and $\Theta_{24}$.

It is quite evident how this is related to type IIB compactifications on K3 modded out by $\Omega \bar{\sigma}$. The key ingredient is the hyperkähler nature of K3 which allows to rotate its complex structure such that holomorphic and special Lagrangian 2-cycles get exchanged. For simplicity, let us choose a purely imaginary complex structure $\tau^{I}=i$ for the original coordinates $z_{i}$ of $(2.21)$, on which $\bar{\sigma}$ acts by complex conjugation. If we then rotate into

$$
z_{1}^{\prime}=x_{1}-i x_{2}, \quad z_{2}^{\prime}=y_{1}+i y_{2}
$$

and define the new holomorphic 2-form $\Omega_{2}^{\prime}$ and the new Kähler form $J^{\prime}$ as in (2.3), the fixed locus of $\bar{\sigma}$, the location of the O7-planes, becomes a holomorphic cycle $\left\{z_{2}^{\prime}=\right.$ $0,1 / 2, i / 2,1 / 2+i / 2\}$, as well as the fixed locus $\left\{z_{1}^{\prime}=0,1 / 2, i / 2,1 / 2+i / 2\right\}$ of $\Theta \bar{\sigma}$. Therefore, if we were only considering D7-branes parallel to the O7-planes at $z_{1}^{\prime}$ or $z_{2}^{\prime}$ given above, we would have just recovered the T-dual version of the standard $\mathbb{Z}_{2}$ orientifold by identifying $z_{1}^{\prime}=\hat{x}_{1}+i \hat{x}_{3}$ and $z_{2}^{\prime}=\hat{x}_{2}+i \hat{x}_{4}$. The deformation we are going to introduce consists in allowing the D7-branes wrap more general cycles in the K3. The former sLag cycles, defined by (2.29), i.e. $\varphi_{1}=\varphi_{2}=\varphi$, become holomorphic cycles given by $z_{1}^{\prime}=\tan (\varphi) z_{2}^{\prime}+c$ in the new complex structure. Any single such brane always comes with its image under $\bar{\sigma}$ at the opposite relative angle $-\varphi$. As we will see, wrapping brane on invariant cycles, symbolically $\pi_{\varphi}+\pi_{-\varphi}$, does have a nice lift to F-theory.

We cannot redo the entire analysis of [71] but need to collect a number of definitions and notations and the important results. It was found that the orbifold was most directly identified with the appropriate F-theory model after breaking the gauge symmetry down to an $S U(2)^{8} \times S U(2)^{8}$ subgroup via turning on Higgs scalars. We have shown in section 4.1 that this precisely matches the situation where all the RR charges can be canceled locally. Geometrically speaking this amounts to placing D7-branes pairwise on top of each other, which are further identified under one of the $\Theta_{i j}$ with another pair. The holomorphic string coupling constant $\lambda(u, v)=a+i e^{-\phi}$ enters the model as the argument of the modular invariant $j$-function

$$
j(\lambda(u, v))=\frac{4(24 f)^{3}}{4 f^{3}+27 g^{2}}
$$


which defines the Weierstraß model

$$
y^{2}=x^{3}+f(u, v) x+g(u, v)
$$

of the elliptic fiber. The functions $f(u, v)$ and $g(u, v)$, are polynomials of degree $(8,8)$ and $(12,12)$. The vanishing locus of the denominator $\Delta=4 f^{3}+27 g^{2}$ defines the region on the base, where the D7-branes and O7-planes are located. It is required that $j \rightarrow \infty$ nearly everywhere, in order to have small string coupling. The coordinates $u, v$ parameterize the base space $\mathbb{C P}^{1} \times \mathbb{C P}^{1}$. Further, $\tilde{u}_{m}, \tilde{v}_{m}$ were used to denote the location of the O7-planes and $u_{i}, v_{i}$ that of the D7-branes of the original model of [71]. There the D7-brane were only wrapping either one of the two cycles $\pi_{9}=[\operatorname{Fix}(\bar{\sigma})]$ and $\pi_{5}=[\operatorname{Fix}(\Theta \bar{\sigma})]$, where the indices are referring to the D5- and D9-brane charges of the original type I model. Then, the expected form of the discriminant for a configuration with the given gauge group is

$$
\Delta=P_{(8,8)} \prod_{i=1}^{8}\left(u-u_{i}\right)^{2}\left(v-v_{i}\right)^{2}
$$

with eight distinct $A_{1}$ singularities and an undetermined polynomial of bi-degree $(8,8)$. A solution to this problem in terms of $f(u, v)$ and $g(u, v)$ could actually be found. It is given by

$$
P_{(8,8)}=4 C^{3} \prod_{i=1}^{8}\left(u-u_{i}\right)\left(v-v_{i}\right)-9 C^{2} h(u, v)^{2},
$$

with $h$ again defined by

$$
h(u, v)=\prod_{m=1}^{4}\left(u-\tilde{u}_{m}\right)\left(v-\tilde{v}_{m}\right)
$$

and $C$ a constant. This leads to

$$
j(\lambda(u, v)) \sim \Delta^{-1}\left(C \prod_{i=1}^{8}\left(u-u_{i}\right)\left(v-v_{i}\right)-3 h(u, v)^{2}\right)^{3}
$$

By scaling $C \rightarrow 0$ one can reach the small coupling region of type IIB string theory. The degrees of the constituents in $\Delta$ actually have a very intuitive interpretation: The polynomial $P_{(8,8)}$ encodes the location of the $4+4$ O7-planes, each split off into two $A_{1}$ singularities around its classical locus $h(u, v)=0$. This splitting of O-planes into multiple singularities is due to the work of [78,79], an application of the Seiberg-Witten curve. On the other hand, the remaining polynomial of bi-degree $(16,16)$ encodes the location of 
$16+16$ D7-branes. The relative factor of 4 between the multiplicities is made up for by the relative normalization of the charges of O7-planes, $Q_{7}=-8$, and pairs of D7-branes. In this sense, the degree of the polynomials directly relates to the charges.

Let us see, how the model should be modified in order to incorporate D7-branes wrapping more general holomorphic cycles. In analogy to the above, we put two branes on top of each other in each stack and wrap them over cycles of the form $\pi_{a}=r_{a} \pi_{9}+s_{a} \pi_{5}$. Since these cycles lie on top of the orientifold planes, supersymmetry is generically preserved and the total space will be Calabi-Yau. The location of the O7-planes is unaffected anyway. Thus, not only $h(u, v)$ should remain unchanged but the structure of $P_{(8,8)}$ as well. For a general D $7_{a}$-brane wrapped on a cycle $\pi_{a}=r_{a} \pi_{9}+s_{a} \pi_{5}$ we now expect a factor $q_{\left(r_{a}, s_{a}\right)}$ in $\Delta$ of degree $\left(r_{a}, s_{a}\right)$. The tadpole cancellation condition (2.14), translated into

$$
\sum_{a}\left(2 r_{a}, 2 s_{a}\right)=(16,16)
$$

then insures, that $\Delta$ will be of the proper degree $(24,24)$ again,

$$
\Delta=P_{(8,8)}^{\prime} \prod_{a} q_{\left(r_{a}, s_{a}\right)}^{2}
$$

The solution for $j(\lambda(u, v))$ in terms of appropriate $f$ and $g$ is now be given completely analogously by just replacing the factors for the D7-branes in (4.53). Thus, we can obtain a small coupling region by scaling $C \rightarrow 0$ as before, the vanishing locus of $\Delta$ referring to potentially smaller number of branes which intersect in some pattern.

As a check, we can perform a similar analysis as was done in [71]. Two stacks of D7branes labeled by $a, b$ will intersect $\pi_{a} \circ \pi_{b}=r_{a} s_{b}+r_{b} s_{a}$ times on the torus. Upon turning on a scalar Higgs fields in the $\left(\mathbf{2}_{a}, \mathbf{2}_{b}\right)$ representation at all of these intersections the gauge group is broken from $S U(2)^{2}$ to the diagonal $S U(2)$. At each intersection one neutral hypermultiplet survives as a modulus from the $\mathbf{2} \otimes \mathbf{2}=\mathbf{1} \oplus \mathbf{3}$ decomposition. In the Ftheory interpretation this would be described by replacing $q_{\left(r_{a}, s_{a}\right)} q_{\left(r_{b}, s_{b}\right)} \rightarrow q_{\left(r_{a}+r_{b}, s_{a}+s_{b}\right)}$. Counting of the free coefficients in the three polynomials, up to an overall rescaling, before and after the symmetry breaking gives

$$
\left(\left(r_{a}+r_{b}+1\right)\left(s_{a}+s_{b}+1\right)-1\right)-\left(\left(r_{a}+1\right)\left(s_{a}+1\right)-1+\left(r_{b}+1\right)\left(s_{b}+1\right)-1\right)=\pi_{a} \circ \pi_{b},
$$


the expected result. In order to get a geometric intuition it is instructive to look at the most simple example $r_{a}=s_{a}=r_{b}=1, s_{b}=0$. In this case we have

$$
q_{(1,1)} q_{(1,0)} \sim\left(u v+a_{1} v+a_{2} u+a_{3}\right)\left(u+b_{1}\right)=(v+a(u))\left(u+a_{1}\right)\left(u+b_{1}\right)=0 .
$$

This describes two stacks of D7-branes, the intersection point given by $\left(v=-a\left(-b_{1}\right), u=\right.$ $\left.-b_{1}\right)$. This intersection locus is being resolved by adding the new parameter,

$$
q_{(2,1)} \sim(v+a(u))\left(u+a_{1}\right)\left(u+b_{1}\right)+c_{1}=0
$$

In a very similar fashion the location of the orientifold planes gets smoothed out by turning on parameters in $h(u, v)=0$, i.e. deforming like

$$
\left(u-\tilde{u}_{m}\right)\left(v-\tilde{v}_{m}\right)=0 \quad \rightarrow \quad\left(u-\tilde{u}_{m}\right)\left(v-\tilde{v}_{m}\right)+\tilde{a}_{1}=0
$$

It is interesting to note that this geometric realization in F-theory suggests that the D7branes are qualitatively unaffected by the resolution of the orientifold singularities, and vice versa the O7-planes by the resolution of intersections. This provides confidence to trust the perturbative CFT analysis at the orbifold point also after slightly deforming away from it.

\subsubsection{F-theory on Voisin-Borcea Calabi-Yau 3-folds}

The class of Voisin-Borcea Calabi-Yau 3-folds 80,81] provides F-theory backgrounds, for which the spectrum and gauge group of the low energy theory have been computed explicitly [74]. Furthermore, these models have partly been interpreted as type I vacua [66,67]. In principle, they can be generalized orientifold vacua, where the coupling constant may vary on the internal space according to the elliptic fibration. In this section we point out that all the Voisin-Borcea 3-folds satisfy the topological tadpole relation (3.5).

The most essential informations we are going to need have been given in [74], which we refer to for the proper definitions and more details. In general, Voisin-Borcea 3-folds are defined by taking a quotient $\mathcal{M}^{6}=\left(\mathrm{K} 3 \times T^{2}\right) / \sigma$. Here, $\sigma$ reflects the holomorphic 2-form of $\mathrm{K} 3$ and acts as a reflection on $T^{2}$,

$$
\sigma:\left(\Omega_{2}, d z\right) \mapsto\left(-\Omega_{2},-d z\right)
$$

$z$ a coordinate on $T^{2}$. If this action has fixed points, they need to be resolved in order to arrive at a smooth manifold with $S U(3)$ holonomy. This manifold is then elliptically 
fibered over $\mathcal{B}=\mathrm{K} 3 / \sigma$. All these spaces are classified in terms of involutions of K3 surfaces [82], and are usually denoted by three integers $(r, a, \delta)$. After the hyperkähler rotation of the complex structure $\sigma$ becomes a complex conjugation on the K3, which makes this set of models accessible to our methods. In order to check (3.5) we then need the number of tensor multiplets in the effective six-dimensional theory and the self-intersection of the fixed locus of $\sigma$. According to [74], we first have $n_{\mathrm{T}}=h^{(1,1)}(\mathcal{B})-1$.

Let us for the moment restrict to all cases with $(r, a, \delta) \neq(10,10,0)$ or $(10,8,0)$. Excluding the two pathological choices, the fixed locus $\operatorname{Fix}(\sigma) \subset \mathcal{B}$ consists of a curve of genus $g=(22-r-a) / 2$ and $k=(r-a) / 2$ rational curves. Using (3.7), we can now easily compute the self-intersection of $\pi_{\mathrm{O} 7}=[\operatorname{Fix}(\sigma)]$,

$$
\pi_{\mathrm{O} 7} \circ \pi_{\mathrm{O} 7}=-\chi(\operatorname{Fix}(\sigma))=2 g-2-2 k=2(10-r) .
$$

This is related to $c_{1}^{2}(\mathcal{B})=10-r$, which via $c_{1}^{2}(\mathcal{B})+c_{2}(\mathcal{B})=12$ allows to deduce $h^{(1,1)}(\mathcal{B})=r$ and, thus, $r=n_{\mathrm{T}}+1$. Hence, all the cases considered satisfy the anomaly cancellation condition derived from the chiral fermion spectrum of table 1. Although it was explicitly checked that they are free of irreducible gravitational anomalies, it is still not automatic that table 1 applies. In principle, it is not even clear, if all the F-theory compactifications on Voisin-Borcea 3-folds have an interpretation as a six-dimensional orientifold. And if so, the F-theory description may involve non-perturbative degrees of freedom, which are beyond any CFT description. In this sense the above analysis can be interpreted as a confirmation that the chiral spectrum of table 1 is indeed valid throughout the moduli space.

The remaining two cases $(r, a, \delta)=(10,10,0)$ and $(10,8,0)$ have been argued to be related to certain orbifolds $T^{6} / \mathbb{Z}_{2} \times \mathbb{Z}_{2}$. For the first choice of $(r, a, \delta), \sigma$ is freely acting. Hence, the two generators $\Theta_{1}$ and $\Theta_{2}$ need to be reflections, defined by $v_{I}=(1 / 2,-1 / 2,0)$ and $v_{I}=(0,1 / 2,-1 / 2)$, combined with shifts of order 2 along $z_{3}$ and $z_{1}$ respectively. Then, $\operatorname{Fix}(\sigma)$ is empty and $n_{\mathrm{T}}=21-n_{\mathrm{H}}=9$. This model has the same spectrum as the Fermat quartic equipped with the involution $\bar{\sigma}$, which was shown to be free as well in section 4.6.

For the second case, only one of the two generators is combined with a shift, the other one given by the reflection. It has 16 fixed $T^{2}$, which are identified pairwise by the free generator. Due to $b_{1}\left(T^{2}\right)=2$, each contributes two scalars which form a hypermultiplet together with their axionic partners. The singularities corresponding to the fixed tori are of type $A_{1}$, leading to a gauge group $U(1)^{8}$. Finally, all the tori are parallel, such that 
$\pi_{\mathrm{O} 7} \circ \pi_{\mathrm{O} 7}=0$, consistent with $n_{\mathrm{T}}=9, n_{\mathrm{H}}=12+8=20$. Still, this model requires D7-branes to cancel the RR charge of the 16 O7-planes. To summarize, all the F-theory vacua on Voisin-Borcea Calabi-Yau 3-fold can consistently be described as generalized six-dimensional orientifolds with intersecting D7-branes, according to the proposed chiral spectrum of table 1 .

\section{Intersecting Brane Worlds in Four Dimensions}

In this section we generalize the results from the previous section to intersecting brane worlds on orientifolds of Calabi-Yau 3-folds.

\subsection{Supersymmetric type II compactifications with D-branes}

The starting point is the type IIA superstring compactified on a Calabi-Yau space $\mathcal{M}^{6}$. The non-trivial Hodge numbers of $\mathcal{M}^{6}$ are denoted by $h^{(1,1)}$ and $h^{(2,1)}$. As it is well known, the closed string sector in four dimensions is built by $\mathcal{N}=2$ supergravity coupled to $h^{(1,1)}$ vector multiplets with gauge group $U(1)^{h^{(1,1)}+1}$. The bosonic degrees of freedom of the vector multiplets are given by the $h^{(1,1)}$ complex Kähler fields $T^{k}$, containing the Kähler deformations of the internal metric $g_{i j}$ plus the internal antisymmetric NSNS fields $b_{i j}$, as well as by $h^{(1,1)} \mathrm{RR}$ vector fields from the 3 -form potential $C_{\mu i j}$. Second, there

arise $h^{(2,1)}+1$ neutral hypermultiplets. More specifically, $h^{(2,1)}$ hypermultiplets are built from $h^{(2,1)}$ complex structure moduli fields $U^{i}$ of $g_{i j}$ plus $h^{(2,1)}$ further complex scalar fields from the RR 3-form potential $C_{i j k}$. The universal hypermultiplet consists out of the dilaton $\phi$, the $b_{\mu \nu} 2$-form plus two RR scalars from $C_{i j k}$.

One important issue about matter coupled to (ungauged) $\mathcal{N}=2$ supergravity is that the moduli space is a product space of the form

$$
\mathcal{M}_{\text {Moduli }}=\mathcal{M}_{\mathrm{V}}\left[h^{(1,1)}\right] \otimes \mathcal{M}_{\mathrm{H}}\left[h^{(2,1)}+1\right]
$$

Due to the special Kähler property, the Kähler potential of $\mathcal{M}_{\mathrm{V}}$ can be expressed as

$$
\mathcal{K}(T, \bar{T})=-\log \left(i \bar{Y}^{K}(\bar{T}) G_{K}(T)-i Y^{K}(T) \bar{G}_{K}(\bar{T})\right)
$$

where the $Y^{K}\left(K=0, \ldots, h^{(1,1)}\right)$ are homogeneous coordinates of $\mathcal{M}_{\mathrm{V}}$ and, at least in a certain symplectic basis, the $G_{K}$ are the first derivatives of a holomorphic prepotential $G(Y)$, i.e. $G_{K}=\partial_{K} G(Y)$. In the so-called special gauge the Kähler moduli of $\mathcal{M}^{6}$ can 
be simply defined as $T^{k}=Y^{k} / Y^{0}\left(k=1, \ldots, h^{(1,1)}\right)$. The holomorphic prepotential is determined by the classical geometry of the Calabi-Yau space $\mathcal{M}^{6}$, namely by the cubic intersection numbers $C_{K L M}$, in addition to all world-sheet, rational (closed string) instantons. However, there are no perturbative string corrections to $\mathcal{M}_{\mathrm{V}}$, since the dilaton belongs to a hypermultiplet.

The couplings and self-interactions of the $h^{(2,1)}+1$ hypermultiplets are determined by the quaternionic space $\mathcal{M}_{\mathrm{H}}$ of (real) dimension $4 h^{(2,1)}+4$. In general its structure is very hard to determine, as $\mathcal{M}_{\mathrm{H}}$ receives string loop corrections (but there are no contributions due to world sheet instantons). However, at least classically, $\mathcal{M}_{\mathrm{H}}$ contains a special Kähler manifold $\widetilde{\mathcal{M}}_{\mathrm{H}}$ of complex dimension $h^{(2,1)}$ as a subspace, as it can be seen from the so-called c-map (time-like T-duality to IIB on the same Calabi-Yau space $\mathcal{M}^{6}$ ). This space $\widetilde{\mathcal{M}}_{\mathrm{H}}$ is parameterized by the complex structure moduli fields $U^{i}\left(i=1, \ldots, h^{(2,1)}\right)$ of $\mathcal{M}^{6}$. Being special Kähler, the same formalism as above applies for $\widetilde{\mathcal{M}}_{\mathrm{H}}$, i.e. there exist a holomorphic prepotential $F(X)$ with corresponding Kähler potential

$$
\mathcal{K}(U, \bar{U})=-\log \left(i \bar{X}^{I}(\bar{U}) F_{I}(U)-i X^{I}(U) \bar{F}_{I}(\bar{U})\right)
$$

with homogeneous fields $X^{I}\left(I=0, \ldots, h^{(2,1)}\right)$ and first derivatives $F_{I}=\partial_{I} F(X)$. As before, in the special Kähler gauge the complex structure moduli are given as $U^{i}=X^{i} / X^{0}$. It is useful to define an integral basis for $H_{3}\left(\mathcal{M}^{6}, \mathbb{Z}\right)$, given by $\left(\alpha^{I}, \beta_{J}\right)\left(I, J=0, \ldots, h^{(2,1)}\right)$ with the property $\alpha^{I} \circ \alpha^{J}=\beta_{I} \circ \beta_{J}=0$ and $\alpha^{I} \circ \beta_{J}=\delta_{J}^{I}$. Sometimes the $\alpha^{I}$ are called electric cycles, and the $\beta_{I}$ are called dual magnetic cycles, however this is clearly a basis dependent statement. Using the holomorphic 3 -form $\Omega_{3}$, one can define the period integrals

$$
X^{I}=\int_{\alpha^{I}} \Omega_{3}, \quad F_{I}=\int_{\beta_{I}} \Omega_{3}
$$

and similarly the normalized periods

$$
\widehat{X}^{I}=\int_{\alpha^{I}} \widehat{\Omega}_{3}, \quad \widehat{F}_{I}=\int_{\beta_{I}} \widehat{\Omega}_{3} .
$$

In fact, the periods (5.4) just correspond to the homogeneous coordinates and the derivatives of the prepotential of the special Kähler geometry introduced before. One can expand every homology 3-cycle $\pi_{a}$ in terms of the basis cycles $\alpha^{I}$ and $\beta_{J}$,

$$
\pi_{a}=e_{I}^{a} \alpha^{I}+m_{a}^{J} \beta_{J}
$$


where the $e_{I}^{a}, m_{a}^{J}$ are integer expansion coefficients.

If we would just consider intersecting D6-branes on the Calabi-Yau manifold, this information would be enough to compute for instance the massless spectrum of chiral fermions. Such models do have $\mathcal{N}=2$ supersymmetry in the bulk and the open string sector does always break supersymmetry completely, which can be seen from the fact that D6-branes always have positive tension which cannot be canceled. If we are interested in models which can preserve an $\mathcal{N}=1$ supersymmetry both in the closed and the open string sector, we have to take an orientifold of the Calabi-Yau where now the world-sheet parity transformation is combined with an anti-holomorphic involution $\bar{\sigma}$. Note, that combining $\Omega$ with a holomorphic involution is not a symmetry of the type IIA string. In fact, $\Omega \bar{\sigma}$ breaks the bulk supersymmetry in the closed string sector from $\mathcal{N}=2$ to $\mathcal{N}=1$. This implies that all bulk $\mathcal{N}=2$ superfields are truncated to $\mathcal{N}=1$ superfields by the $\Omega \bar{\sigma}$ projection. As discussed in section 2.2. there will be $h^{(2,1)}$ complex moduli $U_{i}$, each consisting out of a real complex structure modulus and a linear combination of RR scalars, plus $h^{(1,1)}-a$ complexified Kähler moduli $T_{k}$, where $a$ is the number of the Kähler fields not invariant under $\Omega \bar{\sigma}$. In addition there will be in general $s_{l}(l=1, \ldots, b)$ open string moduli describing the positions of the D-branes. Due to the presence of these fields we do not expect that the total, $\left(h^{(1,1)}+h^{(2,1)}+b-a\right)$-dimensional moduli space has a direct product structure.

The O6-planes are located at the fixed point set of the anti-holomorphic involution, a sLag 3-cycle in the Calabi-Yau manifold. The tadpoles induced by the O6-plane can be canceled by the D6-branes and the positive and negative tensions can potentially compensate each other. In the case that all D6-branes wrap sLag cycles calibrated with respect to the same 3 -form as the orientifold plane is, i.e. $\Re\left(\Omega_{3}\right)$, the background preserves $\mathcal{N}=1$ supersymmetry. It has been established that for such branes on sLag cycles, so-called Atype branes, the $\mathrm{F}$ - and $\mathrm{D}$-terms in the effective action receive the following contributions 31,35: The F-terms, i.e. the superpotential, only depends on the Kähler moduli, getting contributions only from disc and $\mathbb{R I P}^{2}$ instantons. The D-terms, including the FayetIliopoulos term, only depends on the complex structure moduli. In section 2 we have determined the disc level scalar potential and have seen explicitly that it only depends on the complex structure moduli. Therefore, it is clear, that in the effective theory this term appears as a Fayet-Iliopoulos term. D-flatness then imposes conditions on some of the complex structure moduli, freezing them dynamically. Under small changes of the Kähler 
moduli the D-term clearly still vanishes, but the F-term may not, as it gets contributions from disc and $\mathbb{R P}^{2}$ instantons. We will come back to this point later.

If such type IIA vacua with configurations of D6-branes and O6-planes do preserve $\mathcal{N}=1$ supersymmetry, one expects that there exists a lift of the type IIA model to M-theory on a singular seven-dimensional compact $G_{2}$ manifold [83, 84, 12, 13]. In fact not very much is known about the explicit construction of such manifolds, but one can consider the construction of supersymmetric intersecting brane world models of D6-branes and O6-planes as an implicit way of determining the expected properties of such singular manifolds.

\subsection{Chiral fermion spectrum}

In order to compute intersection numbers involving the O6-planes it is necessary that we choose generators (not necessarily a basis) for $H_{3}\left(\mathcal{M}^{6}, \mathbb{Z}\right)$, which allows us to determine both the homology class $\pi_{\mathrm{O} 6}$ of the orientifold plane and the action of $\Omega \bar{\sigma}$ on this basis. As in the K3 case, blown-up orbifold models do naturally give rise to such a basis by combining the inherited toroidal and the exceptional cycles. The intersection matrix for the 3 -cycles is anti-symmetric, so that all self-intersection numbers vanish. Since $Q_{6}=-4$, the RR-tadpole cancellation condition (2.14) reads

$$
\sum_{a} N_{a}\left(\pi_{a}+\pi_{a}^{\prime}\right)=4 \pi_{\mathrm{O} 6}
$$

The gauge group in the generic case, when no D6-brane is invariant under $\Omega \bar{\sigma}$, is again given by (2.15). The spectrum of left-handed massless chiral fermions is shown in table 9 . It is again motivated by computations at the orbifold limit and at large volume, as well as by the general consideration on the topological nature of the zero-modes of the Dirac operator.

\begin{tabular}{|c|c|}
\hline Representation & Multiplicity \\
\hline$\left[\mathbf{A}_{\mathbf{a}}\right]_{L}$ & $\frac{1}{2}\left(\pi_{a}^{\prime} \circ \pi_{a}+\pi_{\mathrm{O} 6} \circ \pi_{a}\right)$ \\
\hline$\left[\mathbf{S}_{\mathbf{a}}\right]_{L}$ & $\frac{1}{2}\left(\pi_{a}^{\prime} \circ \pi_{a}-\pi_{\mathrm{O} 6} \circ \pi_{a}\right)$ \\
\hline$\left[\left(\overline{\mathbf{N}}_{\mathbf{a}}, \mathbf{N}_{\mathbf{b}}\right)\right]_{L}$ & $\pi_{a} \circ \pi_{b}$ \\
\hline$\left[\left(\mathbf{N}_{\mathbf{a}}, \mathbf{N}_{\mathbf{b}}\right)\right]_{L}$ & $\pi_{a}^{\prime} \circ \pi_{b}$ \\
\hline
\end{tabular}

Table 9: Chiral spectrum in $d=4$ 
We therefore claim, that table 9 holds for every smooth six-dimensional background space including Calabi-Yau manifolds and the six-dimensional torus [73]. Note that in contrast to the six-dimensional case, there are no chiral fields in the adjoint representation of $U\left(N_{a}\right)$, as the self-intersection numbers vanish. Computing the non-abelian gauge anomaly $S U\left(N_{a}\right)^{3}$ one finds

$$
A_{\text {non-abelian }} \sim \pi_{a} \circ \pi_{a}=0
$$

Thus, there is no independent check of the construction by testing the resulting spectra for anomalies.

In order to avoid confusion we would like to add the following comment. As for instance pointed out in [31], due to the generation of an $\mathcal{N}=1$ superpotential, it is not clear whether D-branes can be followed smoothly from the large radius regime to regions deep in the interior of the Kähler moduli space. In view of this, we are surely not stating that the spectrum is invariant all over the moduli space. If we go to a point in moduli space where an exact conformal field theory description is available, one can compute the overlap of the corresponding boundary states respectively cross-cap states [85, 31] and use them to define a stringy version of the intersection form. On the other hand, in the large volume regime the methods of classical geometry are available. When passing from one regime to another, the formerly stable configurations may decay into new ones and the spectrum and gauge group changes. But the important point is, whenever the setting is describable by a selection of D-branes on cycles of middle dimension and with flat gauge bundle, table 9 may apply.

\subsection{Background 3-form flux}

To close this section we comment on the possibility to turn on background fluxes ${ }^{3}$, as well. Due to the couplings

$$
\int_{\mathbb{R}^{1,3} \times \mathcal{M}^{6}} H_{3}^{\mathrm{NS}} \wedge G_{6-2 n} \wedge C_{2 n+1}
$$

in the ten-dimensional type IIA string theory, there can exist one more source for the RR 7-form gauge field,

$$
\int_{\mathbb{R}^{1,3} \times \mathcal{M}^{6}} G_{0} H_{3}^{\mathrm{NS}} \wedge C_{7} .
$$

3 Type II models with different sources of RR flux in four dimensions have been discussed e.g. in $86-101$. 
Here, $H_{3}^{\mathrm{NS}}$ is the NSNS 3-form and $G_{0}$ the RR 0-form field strength, which can be considered as a cosmological constant. This coupling induces the term

$$
G_{0}\left[H_{3}^{\mathrm{NS}}\right]
$$

on the left hand side of the RR-tadpole cancellation condition (5.7), where $\left[H_{3}^{\mathrm{NS}}\right]$ denotes the Poincaré dual 3-cycle of the cohomology class of $H_{3}^{\mathrm{NS}}$. Thus, by turning on a nonvanishing NSNS 3-form flux, more general configurations of intersecting D6-branes can satisfy the RR-tadpole cancellation conditions. Anomaly cancellation in such settings has been discussed in [98], and the possibility that such couplings allow a new solution of the strong $C P$ problem has been pointed out in [27]. An important result of the analysis of the anomaly inflow into the brane world volume, which is generated by (5.9), concerns the chiral matter. In fact, the anomaly cancellation in the presence of such background fluxes appears to work out without adding extra contribution from chiral fermions on the world volume of the D6-branes. Therefore, the spectrum of table 9 may be assumed to cover these cases just as well.

However turning on $0 \neq H_{3}^{N S} \in H^{3}(X, \mathbb{R})$ will change the theory drastically if one goes away from the large volume limit where the supergravity description is valid. The NSNS $B$-field should then be thought of as a connection on a non-trivial gerbe, and this will modify the K-theory groups that classify the charges. Especially if the characteristic class of this gerbe is not torsion then there is no Chern isomorphism with ordinary (co)homology any more. This means that the homology class of a brane configuration is no longer an invariant, as was first discovered in the context of WZW models in [102]. This can be understood as D-brane instantons [103], where the instanton background violates the usual conservation of the D-brane homology class.

\section{The Green-Schwarz mechanism}

We have seen that the non-abelian gauge anomalies of all $S U\left(N_{a}\right)$ factors in the gauge group vanish, given the chiral spectrum of table 9 . On the other hand, the mixed anomalies, denoted $U(1)_{a}-S U\left(N_{b}\right)^{2}$, naively do not and a generalized Green-Schwarz mechanism has to be invoked. Computing the $U(1)_{a}-S U\left(N_{b}\right)^{2}$ anomaly in the effective four-dimensional gauge theory for $a \neq b$ one finds

$$
A_{a b}^{(1)}=\frac{N_{a}}{2}\left(-\pi_{a}+\pi_{a}^{\prime}\right) \circ \pi_{b}
$$


For $a=b$ one gets

$$
A_{a a}^{(1)}=\left(\frac{N_{a}-2}{2}\right) 2 \pi_{\mathrm{O} 6} \circ \pi_{a}+\left(\frac{N_{a}}{2}\right) 2\left(\pi_{a}^{\prime} \circ \pi_{a}-\pi_{\mathrm{O} 6} \circ \pi_{a}\right)-\frac{1}{2} \sum_{b \neq a} N_{a} \pi_{a} \circ\left(\pi_{b}+\pi_{b}^{\prime}\right),
$$

where we have used the fact that the $U(1)$ charge of the symmetric and anti-symmetric representations of $U(N)$ are \pm 2 . Using the tadpole cancellation condition the above expression can be simplified to precisely the form (6.1) for $b=a$.

The Green-Schwarz mechanism works quite analogously as in [5]. On each D6 $6_{a}$-brane there exist Chern-Simons couplings of the form

$$
\int_{\mathbb{R}^{1,3} \times \pi_{a}} C_{3} \wedge \operatorname{Tr}\left(F_{a} \wedge F_{a}\right), \quad \int_{\mathbb{R}^{1,3} \times \pi_{a}} C_{5} \wedge \operatorname{Tr}\left(F_{a}\right)
$$

where $F_{a}$ denotes the gauge field on the $\mathrm{D} 6_{a}$-brane. Now we expand every 3 -cycle $\pi_{a}$ and $\pi_{a}^{\prime}$ in the basis $\left(\alpha^{I}, \beta_{J}\right)$

$$
\begin{aligned}
& \pi_{a}=e_{I}^{a} \alpha^{I}+m_{a}^{J} \beta_{J}, \\
& \pi_{a}^{\prime}=\left(e_{I}^{a}\right)^{\prime} \alpha^{I}+\left(m_{a}^{J}\right)^{\prime} \beta_{J} .
\end{aligned}
$$

Moreover, we define the four-dimensional axions $\Phi_{I}$ and 2 -form $B^{I}, I=0, \ldots, h^{(2,1)}$, as

$$
\begin{array}{ll}
\Phi_{I}=\int_{\alpha^{I}} C_{3}, & \Phi^{I+h^{(2,1)}+1}=\int_{\beta_{I}} C_{3}, \\
B^{I}=\int_{\beta_{I}} C_{5}, & B_{I+h^{(2,1)}+1}=\int_{\alpha^{I}} C_{5} .
\end{array}
$$

In four dimensions $\left(d \Phi_{I}, d B^{I}\right)$ and $\left(d \Phi^{I+h^{(2,1)}+1}, d B_{I+h^{(2,1)}+1}\right)$ are Hodge dual to each other. The general couplings (6.3) can now be expressed as

$$
\begin{aligned}
\int_{\mathbb{R}^{1,3} \times \pi_{a}} C_{3} \wedge \operatorname{Tr}\left(F_{a} \wedge F_{a}\right)= & \sum_{I}\left(e_{a}^{I}+\left(e_{a}^{I}\right)^{\prime}\right) \int_{\mathbb{R}^{1,3}} \Phi_{I} \wedge \operatorname{Tr}\left(F_{a} \wedge F_{a}\right) \\
& +\sum_{I}\left(m_{I}^{a}+\left(m_{I}^{a}\right)^{\prime}\right) \int_{\mathbb{R}^{1,3}} \Phi^{I+h^{(2,1)}+1} \wedge \operatorname{Tr}\left(F_{a} \wedge F_{a}\right), \\
\int_{\mathbb{R}^{1,3} \times \pi_{a}} C_{5} \wedge \operatorname{Tr}\left(F_{a}\right)= & N_{a} \sum_{I}\left(m_{I}^{a}-\left(m_{I}^{a}\right)^{\prime}\right) \int_{\mathbb{R}^{1,3}} B^{I} \wedge F_{a} \\
& +N_{a} \sum_{I}\left(e_{a}^{I}-\left(e_{a}^{I}\right)^{\prime}\right) \int_{\mathbb{R}^{1,3}} B_{I+h^{(2,1)}+1} \wedge F_{a},
\end{aligned}
$$




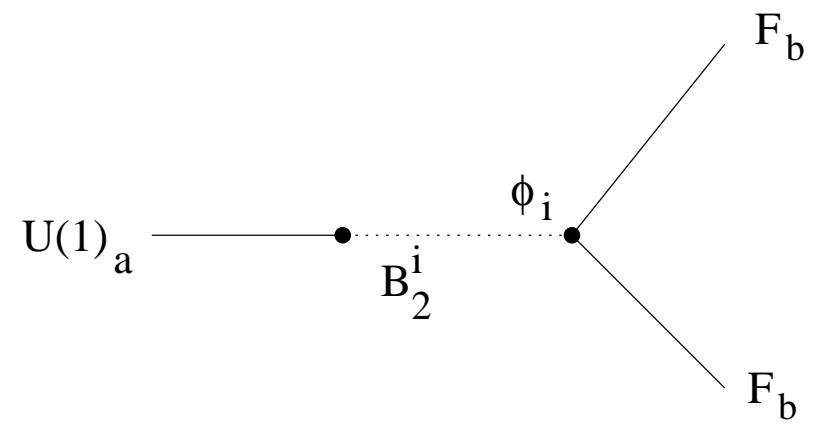

Figure 5

where we have used that the gauge field on the $\Omega \bar{\sigma}$ mirror brane is $F_{a}^{\prime}=-F_{a}$. The treelevel contribution to the mixed gauge anomaly described by these couplings takes the form depicted in figure 5 .

Adding up all terms for the $U(1)_{a}-S U\left(N_{b}\right)^{2}$ anomaly we get

$$
\begin{aligned}
A_{a b}^{(2)} & \sim N_{a} \sum_{I}\left(e_{a}^{I}+\left(e_{a}^{I}\right)^{\prime}\right)\left(m_{I}^{b}-\left(m_{I}^{b}\right)^{\prime}\right)+N_{a} \sum_{I}\left(m_{I}^{a}+\left(m_{I}^{a}\right)^{\prime}\right)\left(e_{b}^{I}-\left(e_{b}^{I}\right)^{\prime}\right) \\
& \sim 2 N_{a}\left(\pi_{a}-\pi_{a}^{\prime}\right) \circ \pi_{b}
\end{aligned}
$$

which has just the right form to cancel the anomalous contribution (6.1) of the chiral fermions.

\section{Examples on Calabi-Yau 3-folds}

Here, we collect examples of intersecting brane worlds in four dimensions. They are given by orbifolds and the algebraic realization of the quintic in $\mathbb{C P}^{4}$.

\subsection{Preliminaries on Calabi-Yau orbifolds}

There is a certain number of orbifolds of $T^{6}$ preserving supersymmetry in four dimensions. These have been classified in [64,65]. The construction of orbifold examples is very similar to the $6 \mathrm{D}$ case. Therefore, we restrict ourselves to the study of only three examples, namely the $\mathbb{Z}_{2} \times \mathbb{Z}_{2}$, the $\mathbb{Z}_{3}$ and the $\mathbb{Z}_{4}$ orbifolds. For the first two examples all 3-cycles are inherited from the $T^{6}$, where for the $\mathbb{Z}_{2} \times \mathbb{Z}_{2}$ orbifold one gets $h^{(2,1)}=3$ and for the $\mathbb{Z}_{3}$ orbifold $h^{(2,1)}=0$.

Such orbifolds are defined by $v_{I}=(1 / N, 1 / N,-2 / N)$ for the two $\mathbb{Z}_{N}$ and by $v_{I}^{(1)}=$ $(1 / 2,-1 / 2,0)$ and $v_{I}^{(2)}=(0,1 / 2,-1 / 2)$ for the two generators of $\mathbb{Z}_{2}^{2}$. Note, that the 
ordinary $\Omega$ orientifolds of type I string theory, e.g. discussed in [104, 105, 106, 107, 47, are T-dual to $\Omega \bar{\sigma}$ orientifolds of asymmetric orbifolds and are not considered in this paper.

We shall employ completely analogous conventions as in the case of six-dimensional models to denote the orbifold backgrounds [108, 109, 110]. The background tori $T^{6}$ for the groups $\mathbb{Z}_{2}^{2}$ and $\mathbb{Z}_{4}$ will be defined by a factorized $S U(2)^{6}$ lattice, while the $\mathbb{Z}_{3}$ torus is given by the $S U(3)^{3}$ lattice. On any single $T_{I}^{2}$ there can be a distinction of two in general inequivalent models according to the complex structures $\tau_{\mathbf{A}}$ and $\tau_{\mathbf{B}}$. This results in a total of six models, $\{\mathbf{A A A}, \mathbf{A B A}, \mathbf{B B A}, \mathbf{A A B}, \mathbf{A B B}, \mathbf{B B B}\}$, for the $\mathbb{Z}_{N}$ and the four $\{\mathbf{A A A}, \mathbf{A A B}, \mathbf{A B B}, \mathbf{B B B}\}$ for $\mathbb{Z}_{2}^{2}$. Actually, we are not going to elaborate all the examples in detail, but simply look for special cases to illustrate the construction. We again pick a basis of 3-cycles on the torus by tensoring the 1-cycles $\gamma_{i}$, which are defined by the basis vectors $\mathbf{e}_{i}^{I}$ of the $\mathbf{A}$ type elementary cells. As in (4.6) we define

$$
\bar{\pi}_{i j k}=\gamma_{i} \otimes \gamma_{j} \otimes \gamma_{k}
$$

The 3-cycles on the orbifold are then given by taking the independent orbits of the $\bar{\pi}_{i j k}$ under the orbifold generators.

At the orbifold point, where the complete CFT description is available, not only the Dterm but also the F-term vanishes in the case of supersymmetric configurations. However, blowing up the orbifold singularities might not be a flat direction of the exact potential, as new non-trivial discs and $\mathbb{R P}^{2}$ appear in the background which might lead to nonvanishing contributions to the superpotential via open string world sheet instantons. We shall discuss the corrections to the superpotential and the D-terms later in some more detail.

\subsection{The orbifold $T^{6} / \mathbb{Z}_{2} \times \mathbb{Z}_{2}$}

In this case the number of 3 -cycles is given by $b_{3}=2+2 h^{(2,1)}=8$. They can be represented by $\left\{\pi_{135}, \pi_{246}, \pi_{245}, \pi_{136}, \pi_{236}, \pi_{145}, \pi_{146}, \pi_{235}\right\}$, which have the intersection form

$$
I_{T^{4} / \mathbb{Z}_{2} \times \mathbb{Z}_{2}}^{\text {Torus }}=\bigoplus_{k=1}^{4}\left(\begin{array}{cc}
0 & 4 \\
-4 & 0
\end{array}\right)_{k} .
$$

For the four different orientifolds the homology classes of the O6-planes are summarized in table 10 . 


\begin{tabular}{|c|c|}
\hline Complex structure & $\pi_{\mathrm{O} 6}$ \\
\hline AAA & $2\left(\pi_{135}+\pi_{245}+\pi_{146}+\pi_{236}\right)$ \\
\hline AAB & $\pi_{135}+\pi_{246}+\pi_{136}+\pi_{245}+\pi_{235}-\pi_{146}+\pi_{145}-\pi_{236}$ \\
\hline ABB & $\pi_{135}-\pi_{246}+\pi_{235}+\pi_{146}$ \\
\hline BBB & $\pi_{135}+\pi_{246}$ \\
\hline
\end{tabular}

Table 10: $\quad T^{6} / \mathbb{Z}_{2} \times \mathbb{Z}_{2}$ orientifolds

The action of $\bar{\sigma}$ on the basis for the homology lattice is evident, regarding earlier results for the six-dimensional $T^{4} / \mathbb{Z}_{2}$ orbifold of section 4.1. Using these data one can construct a variety of intersecting brane world models. A couple of explicit examples including a quasi-realistic three-generation supersymmetric Standard Model can be found in [12, 13, 28]. There, the computation was performed in the orbifold limit and the spectra were computed by some effort in solving constraints for the Chan-Paton matrices. Actually, the results for the massless spectra precisely match with table 9 .

\subsection{The orbifold $T^{6} / \mathbb{Z}_{3}$}

The orbifold $T^{6} / \mathbb{Z}_{3}$ has Hodge numbers $\left(h^{(1,1)}, h^{(2,1)}\right)=(36,0)$, so that the number of 3 -cycle is $b_{3}=2$. A basis is given by

$$
\begin{aligned}
& \pi_{1}=\bar{\pi}_{136}+\bar{\pi}_{145}+\bar{\pi}_{235}-\bar{\pi}_{146}+\bar{\pi}_{236}+\bar{\pi}_{245}, \\
& \pi_{2}=\bar{\pi}_{135}+\bar{\pi}_{246}-\bar{\pi}_{136}-\bar{\pi}_{145}+\bar{\pi}_{235} .
\end{aligned}
$$

It leads to the intersection matrix

$$
I_{T^{6} / \mathbb{Z}_{3}}^{\text {Torus }}=\left(\begin{array}{cc}
0 & 1 \\
-1 & 0
\end{array}\right) .
$$

For the choice AAA of the complex structure the homology class of the orientifold plane is

$$
\pi_{\mathrm{O} 6}=\pi_{1},
$$

and the action of $[\bar{\sigma}]_{\mathbf{A A A}}$ on $H_{3}\left(T^{6} / \mathbb{Z}_{3}, \mathbb{Z}\right)$ reads

$$
[\bar{\sigma}]_{\mathbf{A A A}}=\left(\begin{array}{cc}
1 & 0 \\
1 & -1
\end{array}\right) .
$$


We skip the computation for the other choices of complex structure. Instead, we show that one can indeed recover the 3 generation $S U(5)$ GUT model found in [11] as a $T^{6} / \mathbb{Z}_{3}$ orbifold compactification. Choosing $N_{1}=5$ and $N_{2}=1$ D6-branes wrapped on the cycles

$$
\begin{array}{ll}
N_{1}=5: & \eta_{1}=-\pi_{1}+3 \pi_{2}, \\
N_{1}=5: & \eta_{1}^{\prime}=2 \pi_{1}-3 \pi_{2}, \\
N_{2}=1: & \eta_{2}=-2 \pi_{1}+3 \pi_{2}, \\
N_{2}=1: & \eta_{2}^{\prime}=\pi_{1}-3 \pi_{2} .
\end{array}
$$

These branes support a gauge group $U(5) \times U(1)$. Computing the relevant intersection numbers we obtain the chiral spectrum

\begin{tabular}{|c|c|}
\hline Representation & Multiplicity \\
\hline$[(\mathbf{1 0}, \mathbf{0})+(\mathbf{1}, \mathbf{- 2})]_{L}$ & 3 \\
{$[(\overline{\mathbf{5}}, \mathbf{1})]_{L}$} & 3 \\
\hline
\end{tabular}

Table 11: $\mathbb{Z}_{3}$ orientifolds

which agrees with the spectrum found in [1]. There, the gauge symmetry breaking patterns, the perturbative stability of the model and some phenomenological implications have been studied.

\subsection{The orbifold $T^{6} / \mathbb{Z}_{4}$}

In this case, there are four 3-cycles inherited from the $T^{6}$ corresponding to one untwisted complex structure deformation in the orbifold model. A basis is given by

$$
\begin{aligned}
& \pi_{1}=2 \bar{\pi}_{135}-2 \bar{\pi}_{245}, \\
& \pi_{2}=2 \bar{\pi}_{136}-2 \bar{\pi}_{246}, \\
& \pi_{3}=2 \bar{\pi}_{145}+2 \bar{\pi}_{235}, \\
& \pi_{4}=2 \bar{\pi}_{146}+2 \bar{\pi}_{236},
\end{aligned}
$$

with the intersection matrix

$$
I_{T^{6} / \mathbb{Z}_{4}}^{\text {Torus }}=\bigoplus_{k=1}^{2}\left(\begin{array}{cc}
0 & -2 \\
2 & 0
\end{array}\right)_{k} .
$$


Let us just present one example for an ABA model, which has a supersymmetric groundstate. In this case, the O6-plane is wrapping the 3-cycle

$$
\pi_{\mathrm{O} 6}=2\left(\pi_{1}+\pi_{2}+\pi_{3}-\pi_{4}\right) .
$$

Introducing the four stacks of D6-branes

$$
\begin{array}{ll}
N_{1}=2: & \eta_{1}=3\left(\pi_{1}+\pi_{2}\right)-\left(\pi_{3}+\pi_{4}\right), \\
N_{1}=2: & \eta_{1}^{\prime}=3\left(\pi_{3}-\pi_{4}\right)-\left(\pi_{1}-\pi_{2}\right), \\
N_{2}=2: & \eta_{2}=\pi_{1}+\pi_{3}, \\
N_{2}=2: & \eta_{2}^{\prime}=\pi_{1}+\pi_{3}
\end{array}
$$

cancels the RR-tadpole. the gauge group given by $U(2)^{2}$. Computing the intersection numbers leads to the spectrum

\begin{tabular}{|c|c|}
\hline Representation & Multiplicity \\
\hline$[(\mathbf{A}, \mathbf{1})]_{L}$ & 16 \\
{$[(\mathbf{S}, \mathbf{1})]_{L}$} & 8 \\
{$[(\overline{\mathbf{2}}, \mathbf{2})]_{L}+[(\mathbf{2}, \overline{\mathbf{2}})]_{L}$} & 4 \\
\hline
\end{tabular}

Table 12: $\mathbb{Z}_{4}$ orientifolds

which is, of course, free of non-abelian gauge anomalies. We now fix the complex structures of the first two $T_{I}^{2}$ and allow $\Im\left(\tau^{3}\right)=U$ to vary. At the orbifold point, the scalar potential reads

$$
\mathcal{V}=T_{6} e^{-\phi_{4}}\left[16 \sqrt{2 U}+16 \sqrt{10\left(U+\frac{1}{U}\right)}-32 \sqrt{2}\left(\sqrt{U}+\frac{1}{\sqrt{U}}\right)\right]
$$

which can be shown to be positive, $\mathcal{V} \geq 0$. It vanishes for $U=1 / 2$. Thus, we have found another $\mathcal{N}=1$ supersymmetric intersecting brane world model which should lift in M-theory to a $G_{2}$ manifold.

\subsection{The Quintic}

As an example of a Calabi-Yau manifold that is not a torus orbifold consider the Fermat quintic $Q$

$$
\sum_{i=1}^{5} z_{i}^{5}=0 \quad \subset \mathbb{C P}^{4}
$$


together with the obvious involution from the complex conjugation of the coordinates $z_{i} \rightarrow \bar{z}_{i}$. The fixed points are the real quintic $\sum_{i=1}^{5} x_{i}^{5}=0 \subset \mathbb{R P}^{4}$. Topologically this is a sLag $\mathbb{R I P}^{3}$.

Now the Fermat quintic has $\mathbb{Z}_{5}^{5}$ acting on it via

$$
z_{i} \mapsto \omega^{k_{i}} z_{i} \quad \omega=e^{\frac{2 \pi i}{5}}, k_{i} \in \mathbb{Z}_{5}
$$

The diagonal $\mathbb{Z}_{5}$ acts trivially, therefore we get $5^{4}=625$ different minimal $\mathbb{R} \mathbb{P}^{3} \mathrm{~s}$

$$
\left|k_{2}, k_{3}, k_{4}, k_{5}\right\rangle \stackrel{\text { def }}{=}\left\{\left[x_{1}: \omega^{k_{2}} x_{2}: \omega^{k_{3}} x_{3}: \omega^{k_{4}} x_{4}: \omega^{k_{5}} x_{5}\right] \mid x_{i} \in \mathbb{R}, \sum_{i=1}^{5} x_{i}^{5}=0\right\}
$$

The intersection number of $|1,1,1,1\rangle$ and $\left|k_{2}, k_{3}, k_{4}, k_{5}\right\rangle$ was determined in [31], it can be written as the coefficient of the monomial $g_{2}^{k_{2}} g_{3}^{k_{3}} g_{4}^{k_{4}} g_{5}^{k_{5}}$ in

$$
I_{\mathbb{R} \mathbb{P}^{3}}=\prod_{i=1}^{5}\left(g_{i}+g_{i}^{2}-g_{i}^{3}-g_{i}^{4}\right) \in \mathbb{Z}\left[g_{1}, g_{2}, g_{3}, g_{4}, g_{5}\right] /\left\langle g_{i}^{5}=1, \prod g_{i}=1\right\rangle
$$

All the other intersection numbers are then determined by the $\mathbb{Z}_{5}^{4}$ symmetry. The ensuing intersection matrix $M \in \operatorname{Mat}(625, \mathbb{Z})$ has rank $204=b_{3}$, so the $\left|k_{2}, k_{3}, k_{4}, k_{5}\right\rangle$ generate the full $H_{3}(Q ; \mathbb{R})$.

Of course, not all those minimal $\mathbb{R P}^{3}$ are $\Re\left(\Omega_{3}\right)$ calibrated, but rather $\Re\left(\omega^{k} \Omega_{3}\right)$ calibrated. Indeed the $\Re\left(\Omega_{3}\right)$ calibrated sLag cycles $L$ cannot span $H_{3}(Q)$ since $\left.\Im\left(\Omega_{3}\right)\right|_{L}=$ $0 \Rightarrow L$ is orthogonal to $\operatorname{PD}\left(\Im\left(\Omega_{3}\right)\right)$. To determine the $\Re\left(\Omega_{3}\right)$ sLags we need to know how $\mathbb{Z}_{5}^{4}$ acts on the holomorphic volume form. This is clear from the residue formula

$$
2 \pi i \Omega_{3}=\oint_{\Gamma} \frac{\epsilon^{i_{1} \cdots i_{5}} z_{i_{1}} \mathrm{~d} z_{i_{2}} \wedge \mathrm{d} z_{i_{3}} \wedge \mathrm{d} z_{i_{4}} \wedge \mathrm{d} z_{i_{5}}}{\sum_{i=1}^{5} z_{i}^{5}}
$$

So $\Omega_{3}$ transforms as $\Omega_{3} \mapsto\left(\prod_{i} \omega^{k_{i}}\right) \Omega_{3}$, and the $\Re\left(\Omega_{3}\right)$ calibrated $\mathbb{R P}^{3}$ s are the $\left|k_{2}, k_{3}, k_{4}, k_{5}\right\rangle$ with $\sum_{i=2}^{5} k_{i}=0 \bmod 5$. There are $\frac{1}{5} 5^{4}=125$ such sLags, and using the intersection matrix one can check that they generate a 101-dimensional subspace of $H_{3}(Q)$. Furthermore those 125 cycles have vanishing intersection numbers among themselves, so by wrapping branes on these sLag cycles one cannot obtain chiral fermions.

Even without chiral fermions, is it at least possible to find supersymmetric tadpolefree models besides the one with all branes sitting on top of the orientifold plane? First consider the RR tadpole. Since the $125 \mathrm{sLag} \mathbb{R P}^{3}$ s span only a 101-dimensional subspace of $H_{3}(Q)$ there are 24 relations between them. This yields a 24 dimensional family of 
brane configurations that formally cancel the RR tadpole (2.14). But even if the overall homology class adds up correctly we have to make sure that we use only branes, and no antibranes (whether some cycle is a brane or antibrane is determined by its orientation relative to the orientation induced by the calibration). So in the 24 dimensional space of relations we may only use the positive coefficients if we want to preserve supersymmetry. It can easily be seen that there is no solution besides the zero relation (for example note that the coefficients in the relations add up to 0 ).

Secondly let us consider the NSNS tadpole. For this we need to know the volume of some linear combination of the sLags. But because we got all the $\mathbb{R P}^{3}$ s by acting with a symmetry they all have the same volume (say, 1):

$$
\operatorname{Vol}\left(\sum_{a=1}^{125} n_{a}\left|k_{2}^{(a)}, k_{3}^{(a)}, k_{4}^{(a)}, k_{5}^{(a)}\right\rangle\right)=\sum_{a=1}^{125}\left|n_{a}\right|
$$

So let $\sum \tilde{n}_{a}\left|k_{2}^{(a)}, k_{3}^{(a)}, k_{4}^{(a)}, k_{5}^{(a)}\right\rangle$ be an arbitrary (nonzero) relation, i.e. homologous to zero, and let $\tilde{n}_{1}$ be the coefficient of $|1,1,1,1\rangle$. Then it is not difficult ${ }^{4}$ to see that

$$
\left|Q+\tilde{n}_{1}\right|+\sum_{a=2}^{125}\left|\tilde{n}_{a}\right|>|Q|
$$

So the volume of the cycle always increases if we go away from the configuration with all branes wrapping the orientifold plane. This means that there is also no NSNS tadpole free configuration besides the trivial one, at least not without introducing other sLag cycles besides the $125 \mathbb{R P}^{3}$ s.

\subsection{The Quintic Standard Model}

We have seen that using the 3-cycles in (7.15) we cannot obtain interesting brane configurations if we want to preserve supersymmetry. However one might hope that one could give up supersymmetry and then at least realize the Standard Model with intersecting branes. This is indeed possible, as we will show in this section.

First note that all intersection numbers of the 625 minimal $\mathbb{R I P}^{3}$ s are in the range $-2, \ldots, 2$, while we need \pm 3 for some cycles. So we cannot simply wrap branes on the

4 For example, note that there is an integral basis for the relations such that the coefficient of $|1,1,1,1\rangle$ is always $-1,0$, or 1 , and such that for each of the 24 generators there is some $\mathbb{R P}^{3} \mathrm{~s}$ with coefficient \pm 1 that does not occur in the other relations. 
$\mathbb{R P}^{3} \mathrm{~s}$, we must use linear combinations. Fortunately one can represent every class in $H_{3}(Q ; \mathbb{Z})$ by a connected $\operatorname{Spin}^{\mathbb{C}}$ submanifold for dimensional reasons. In addition to the O6-plane on the cycle $\pi_{\mathrm{O} 6}=|0,0,0,0\rangle$ we wrap D6-branes on the following 3-cycles

$$
\begin{aligned}
& \pi_{a}=|0,0,3,1\rangle \quad \pi_{a}^{\prime}=|0,0,2,4\rangle \\
& \pi_{b}=|4,3,0,3\rangle \quad \pi_{b}^{\prime}=|1,2,0,2\rangle \\
& \pi_{c}=|3,0,1,1\rangle-2|4,3,0,3\rangle \quad \pi_{c}^{\prime}=|2,0,4,4\rangle-2|1,2,0,2\rangle \\
& \pi_{d}=|4,2,4,4\rangle-2|0,0,3,1\rangle \quad \pi_{d}^{\prime}=|1,3,1,1\rangle-2|0,0,2,4\rangle
\end{aligned}
$$

It is straightforward to check that the homology classes are primitive, i.e. not a multiple of some other class in $H_{3}(Q, \mathbb{Z})$, by finding one $\mathbb{R}^{3}$ such that the intersection number is \pm 1 . The intersection numbers of the given 3-cycles are shown in table 13 .

\begin{tabular}{|c||c|c|c|c|c|c|c|c|c|}
\hline$\circ$ & $\pi_{a}$ & $\pi_{b}$ & $\pi_{c}$ & $\pi_{d}$ & $\pi_{a}^{\prime}$ & $\pi_{b}^{\prime}$ & $\pi_{c}^{\prime}$ & $\pi_{d}^{\prime}$ & $\pi_{\mathrm{O} 6}$ \\
\hline \hline$\pi_{a}$ & 0 & -1 & 3 & 0 & 0 & -2 & 3 & 0 & 0 \\
\hline$\pi_{b}$ & 1 & 0 & 0 & 0 & -2 & 0 & 0 & 3 & 0 \\
\hline$\pi_{c}$ & -3 & 0 & 0 & 3 & 3 & 0 & 0 & -3 & 0 \\
\hline$\pi_{d}$ & 0 & 0 & -3 & 0 & 0 & 3 & -3 & 0 & 0 \\
\hline$\pi_{a}^{\prime}$ & 0 & 2 & -3 & 0 & 0 & 1 & -3 & 0 & 0 \\
\hline$\pi_{b}^{\prime}$ & 2 & 0 & 0 & -3 & -1 & 0 & 0 & 0 & 0 \\
\hline$\pi_{c}^{\prime}$ & -3 & 0 & 0 & 3 & 3 & 0 & 0 & -3 & 0 \\
\hline$\pi_{d}^{\prime}$ & 0 & -3 & 3 & 0 & 0 & 0 & 3 & 0 & 0 \\
\hline$\pi_{\mathrm{O} 6}$ & 0 & 0 & 0 & 0 & 0 & 0 & 0 & 0 & 0 \\
\hline
\end{tabular}

Table 13: Intersection numbers

Note the symmetries of the intersection matrix

$$
\begin{gathered}
\pi_{i} \circ \pi_{j}=-\pi_{j} \circ \pi_{i}=\pi_{j}^{\prime} \circ \pi_{i}^{\prime}=-\pi_{i}^{\prime} \circ \pi_{j}^{\prime}, \\
\pi_{i} \circ \pi_{j}^{\prime}=\pi_{j} \circ \pi_{i}^{\prime}=-\pi_{i}^{\prime} \circ \pi_{j}=\pi_{j} \circ \pi_{i}^{\prime} .
\end{gathered}
$$

The intersection pattern in table 13 is precisely the one required for the Standard Model as proposed in [8]: If one wraps four different stacks of D6-branes, namely a stack of 3 branes on $\pi_{a}$, a stack of 2 branes on $\pi_{b}$, and a single brane on $\pi_{c}$ and $\pi_{d}$ then the gauge 
group is $U(3) \times U(2) \times U(1)^{2}$; in addition one gets just the necessary bifundamental chiral fermions. Since $\pi_{i} \circ \pi_{i}^{\prime}=\pi_{i} \circ \pi_{\mathrm{O} 6}=0$ there are no chiral fermions in the symmetric or antisymmetric representations of the gauge groups. The chiral massless spectrum is shown in table 14 .

\begin{tabular}{|l|l|l|l|l|}
\hline Sector & Field & $S U(3) \times S U(2) \times U(1)^{4}$ & $U(1)_{Y}$ & Multiplicity \\
\hline$(a b)$ & $Q_{L}$ & $(\mathbf{3}, \mathbf{2})_{(1,-1,0,0)}$ & $1 / 3$ & 1 \\
$\left(a^{\prime} b\right)$ & $Q_{L}$ & $(\mathbf{3}, \mathbf{2})_{(1,1,0,0)}$ & $1 / 3$ & 2 \\
$(a c)$ & $U_{R}$ & $(\overline{\mathbf{3}}, \mathbf{1})_{(-1,0,1,0)}$ & $-4 / 3$ & 3 \\
$\left(a^{\prime} c\right)$ & $D_{L}$ & $(\overline{\mathbf{3}}, \mathbf{1})_{(-1,0,-1,0)}$ & $2 / 3$ & 3 \\
\hline$\left(b^{\prime} d\right)$ & $L_{L}$ & $(\mathbf{1}, \mathbf{2})_{(0,-1,0,-1)}$ & -1 & 3 \\
$(c d)$ & $E_{R}$ & $(\mathbf{1}, \mathbf{1})_{(0,0,-1,1)}$ & 2 & 3 \\
$\left(c^{\prime} d\right)$ & $N_{L}$ & $(\mathbf{1}, \mathbf{1})_{(0,0,1,1)}$ & 0 & 3 \\
\hline
\end{tabular}

Table 14: Chiral left-handed fermions for the 3 generation model.

The anomaly-free hypercharge is given by

$$
U(1)_{Y}=\frac{1}{3} U(1)_{a}-U(1)_{c}+U(1)_{d} .
$$

The phenomenological properties of this model are analogous to the one described in detail in [8].

Of course, so far our model does have a nonvanishing RR tadpole: The sum of the homology classes

$$
\begin{aligned}
\pi & \stackrel{\text { def }}{=} \sum_{a} N_{a}\left(\pi_{a}+\pi_{a}^{\prime}\right)+Q_{q} \pi_{O q}= \\
& =3\left(\pi_{a}+\pi_{a}^{\prime}\right)+2\left(\pi_{b}+\pi_{b}^{\prime}\right)+\left(\pi_{c}+\pi_{c}^{\prime}\right)+\left(\pi_{d}+\pi_{d}^{\prime}\right)-4 \pi_{\mathrm{O} 6}
\end{aligned}
$$

does not vanish. For example, one can easily find other cycles that have nonzero intersection with $\pi$. However the intersection number of $\pi$ and the standard model branes does vanish. This is not surprising since the intersection numbers were precisely chosen to yield the Standard Model so that this set of intersecting branes yields an anomaly-free spectrum by themselves.

Thus, one can introduce a hidden brane that carries the right charge to cancel the tadpole but does not intersect the Standard Model branes, so there is no bifundamental 
matter charged under both the SM and the hidden gauge group. For this we simply add

one brane in the homology class $\pi_{H} \stackrel{\text { def }}{=}-\pi$. As already noted it does not intersect the SM branes, so this is really a hidden brane.

We find it quite amusing that it is fairly easy to construct a non-supersymmetric three generation standard model on the quintic Calabi-Yau. Using different 3-cycles on the quintic it remains to be seen whether it is also possible to construct a realistic supersymmetric intersecting brane model coming as close as possible to the MSSM.

\section{Stability, scalar potential and gauge couplings}

Non-supersymmetric brane configurations are in general unstable. On the one hand, depending on the intersection angles there can be tachyons localized at the intersection points. Phenomenologically it was suggested that these tachyons might be interpreted as Standard Model Higgs fields [111,1], where in particular in [22] it was demonstrated that the gauge symmetry breaking is consistent with this point of view. On the other hand, even if tachyons are absent one generally faces uncanceled NSNS tadpoles, which might destabilize the configuration [20,11,21,24]. In [20] it was shown that it is at least possible for appropriate choices of the D-branes that the complex structure moduli are stabilized by the induced open string tree level potential. The stabilization of the dilaton remains a major challenge as in all non-supersymmetric string models.

For supersymmetric intersecting brane worlds we can expect much better stability properties. First tachyons are absent in these models due to the Bose-Fermi degeneracy. However, since for orientifolds on Calabi-Yau spaces the configuration only preserves $\mathcal{N}=1$ supersymmetry, in general non-trivial F-term and D-term potentials can be generated. In the case that an exact CFT description is available, like in the orbifold examples, it is clear that at that point in moduli space the F- and D-terms vanish, but in general such terms will be generated. Luckily, the generation of D-term and F-term potentials for D-branes wrapping sLag cycles of some underlying Calabi-Yau manifold has been the subject of intense study during the recent years. Therefore, in the remainder of this section we will collect partially known results for the contributions to the F-term and D-term potential. 


\subsection{F-term superpotential}

The effective $\mathcal{N}=1$ superpotential of a type II compactification on a Calabi-Yau 3-fold with D6-branes and O6-planes on sLag cycles receives contributions from different sources. Due to supersymmetry there are no string loop corrections to the superpotential, and the Peccei-Quinn symmetry forbids perturbative $\alpha^{\prime}$ corrections. The only corrections possible arise from world-sheet instantons. They are provided by oriented closed strings with the topology of a sphere, wrapping homologically non-trivial 2-cycles in the CalabiYau manifold, and by unoriented closed strings with the topology of $\mathbb{R P}^{2}$, ending on the orientifold plane [112]. Finally, the superpotential can receive contributions from open strings discs glued to the various D6-branes [113, 114, 115, 43].

It is known that closed string instantons on a type II Calabi-Yau background do not generate a superpotential, so let us discuss the disc instanton corrections a bit more detailed. Consider a D6-brane which is wrapped on a supersymmetric Lagrangian 3-cycle $\Sigma \subset \mathcal{M}^{6}$. In general, all holomorphic open string disc instantons will contribute to the superpotential, which will be given in terms of an instanton sum of the form [38

$$
w\left(s, T^{k}\right)=\sum_{k, n, \vec{m}} \frac{1}{n^{2}} N_{k, \vec{m}} \exp (n[k s-\vec{m} \cdot \vec{T}]) .
$$

Here the open string modulus $s$ parameterizes the size of the holomorphic disc, $N_{k, \vec{m}}$ are integers counting the number of domain wall D4-branes ending on the D6-brane, which wrap a certain 2-cycle class captured by $\vec{m}$, and $k$ finally denotes the wrapping number around the boundary. In the large volume limit, $T^{k} \rightarrow \infty$, the classical superpotential is vanishing. Thus, very similar to heterotic string models with $(0,2)$ world-sheet supersymmetry, also for supersymmetric intersecting brane models one expects the background to be destabilized by world-sheet instanton corrections. However, in the $(0,2)$ context is is known that there exist a subclass of models, the so-called Distler-Kachru models [116], which are not destabilized by world-sheet instantons [117,118, 119, 120. It would be interesting to determine a stable class of $\mathcal{N}=1$ supersymmetric intersecting brane models, as well.

To compute this instanton expansion directly is a horrendous task, as one does not understand the background well enough to determine all non-trivial holomorphic discs. However, the instanton expansion for A-type branes and orientifold planes, D-branes and O-planes on sLag cycles, can be computed in simple cases by the use of mirror symmetry. The contribution from the disc and $\mathbb{R P}^{2}$ world-sheets arise from domain walls in the type 
IIB model [121,122]. Such domain-walls are given by D5-branes and O5-planes wrapping supersymmetric 3 -cycles in $\mathcal{W}^{6}$, the mirror dual of $\mathcal{M}^{6}$. Therefore the superpotential can only depend on the complex structure of $\mathcal{W}^{6}$ and there are no world-sheet instanton corrections on the type IIB side. The superpotential simply reads

$$
w=\int_{\mathcal{W}^{6}} G \wedge \Omega_{3}
$$

where $G=d C_{2}$ is the RR 3-form field strength. Note, that (8.2) is more general in the sense that even without explicit D5-branes all sources for the $\mathrm{RR}$ flux $G$ also contribute to this superpotential. For some non-compact Calabi-Yau 3-folds and certain D6-brane configurations (8.2) has indeed been computed, leading to highly non-trivial results on the number of holomorphic discs in the dual backgrounds $\mathcal{M}^{6}$.

For the particular case we are going to study in section 10, where we consider the mirror situation with D9-branes with gauge fluxes $\mathcal{F}$, the equation of motion for $G$ can be obtained from the formulas given there. It reads

$$
\frac{1}{\kappa^{2}} d G=-\frac{\mu_{9}}{8 \pi^{2}}\left[\sum_{a} 2 n_{a} \operatorname{Tr}\left(\mathcal{F}_{a} \wedge \mathcal{F}_{a}\right)-\operatorname{Tr}\left(\mathcal{R}_{a} \wedge \mathcal{R}_{a}\right)\right]+2 \mu_{5} \delta(\mathrm{O} 5)
$$

Here, $\delta(\mathrm{O} 5)$ denotes a form which is supported at the location of the O5-planes. Note, that (8.3) also includes the contribution from the O9-plane to the curvature term. It can be integrated and using the result from [45] for the O5-plane we get

$$
\begin{aligned}
w=-\frac{\mu_{9} \kappa^{2}}{8 \pi^{2}} & {\left[\sum_{a} \int_{\mathcal{W}^{6}} 2 n_{a} \operatorname{Tr}\left(A_{a} \wedge d A_{a}+\frac{2}{3} A_{a} \wedge A_{a} \wedge A_{a}\right) \wedge \Omega_{3}\right.} \\
& \left.-\int_{\mathcal{W}^{6}} \operatorname{Tr}\left(\omega \wedge d \omega+\frac{2}{3} \omega \wedge \omega \wedge \omega\right) \wedge \Omega_{3}\right]+2 \mu_{5} \kappa^{2} \int_{C} \Omega_{3},
\end{aligned}
$$

where $\omega$ denotes the spin-connection and $C$ a 3-cycle satisfying $\partial C=\pi_{\mathrm{O} 5}$. The superpotential (8.4) looks very similar to the superpotential for the type I or heterotic string. From this discussion it is clear that to really compute the superpotential for the A-type models with intersecting D6-branes it is essential to gain a better understanding of the detailed mirror map for the D6-branes. 


\subsection{D-term potential at tree level}

The self-energy of the D-branes is due to the tension of the wrapped D-branes. Since the open string tree-level scalar potential only depends on the complex structure moduli it is clear that in the effective $\mathcal{N}=1$ field theory this potential does not arise from a holomorphic superpotential but from a D-term. The D-term scalar potential has the following general form

$$
\mathcal{V}_{\mathrm{D}-\text { term }}=\sum_{a} \frac{1}{2 g_{a}^{2}}\left(\sum_{i} q_{a}^{i}\left|\phi_{i}\right|^{2}+\xi_{a}\right)^{2} .
$$

Here $g_{a}$ is the gauge coupling of some $U(1)_{a}$ gauge group, and $\xi_{a}$ is the Fayet-Iliopoulos term associated to $U(1)_{a}$. The scalar fields $\phi_{i}$ will obtain a positive or negative quadratic mass term for non-vanishing $\xi_{a}$, depending on the sign of their $U(1)_{a}$ charges $q_{a}^{i}$. In the intersecting brane worlds the potential energy of the wrapped branes corresponds to the term $\frac{1}{g_{a}^{2}} \xi_{a}^{2}$ in (8.5), as we will discuss in the following, and the scalars $\phi_{i}$ are open strings fields which are massive, massless or tachyonic depending on the choice of moduli parameters. $\mathcal{N}=1$ supersymmetry will be unbroken, if the potential vanishes in the groundstate. In particular this can be achieved for $\xi_{a}=0$, where the scalars $\phi_{i}$ are all

massless. If, however, $\xi_{a}>0$, a supersymmetric groundstate is only accessible after the condensation of a tachyonic scalar.

Recall that the disc level scalar potential for D6-branes wrapping sLag cycles can be written as

$$
\begin{aligned}
\mathcal{V} & =T_{6} e^{-\phi_{4}}\left[\sum_{a} N_{a}\left|\int_{\pi_{a}} \widehat{\Omega}_{3}\right|+\sum_{a} N_{a}\left|\int_{\pi_{a}^{\prime}} \widehat{\Omega}_{3}\right|-4 \int_{\pi_{\mathrm{O} 6}} \Re\left(\widehat{\Omega}_{3}\right)\right] \\
& =2 T_{6} e^{-\phi_{4}} \sum_{a} N_{a}\left(\left|\int_{\pi_{a}} \widehat{\Omega}_{3}\right|-\int_{\pi_{a}} \Re\left(\widehat{\Omega}_{3}\right)\right)
\end{aligned}
$$

where we have used (2.19) and (2.14). On the other hand, the $U(1)_{a}$ gauge coupling is given by

$$
\frac{1}{g_{U(1)_{a}}^{2}}=\frac{N_{a}}{g_{a}^{2}}=\frac{N_{a} M_{s}^{3}}{(2 \pi)^{4}} e^{-\phi_{4}}\left|\int_{\pi_{a}} \widehat{\Omega}_{3}\right| .
$$

Hence, by direct comparison with (8.5) we see that the FI-term can be identified as

$$
\xi_{a}^{2}=\frac{M_{s}^{4}}{2 \pi^{2}}\left(\frac{\left|\int_{\pi_{a}} \widehat{\Omega}_{3}\right|-\int_{\pi_{a}} \Re\left(\widehat{\Omega}_{3}\right)}{\left|\int_{\pi_{a}} \widehat{\Omega}_{3}\right|}\right),
$$


which apparently vanishes if the D-brane is calibrated with respect to the same 3 -form as the orientifold plane.

Since the FI-term is not a holomorphic quantity one expects higher loop corrections to the classical result (8.8). Using the expansion (5.6) of the 3-cycle $\pi_{a}$ in terms the basis 3-cycles $\alpha^{I}$ and $\beta_{J}$, one can write

$$
\left|\int_{\pi_{a}} \widehat{\Omega}_{3}\right|=\left|e_{I}^{a} \hat{X}^{I}(U)-m_{a}^{I} \hat{F}_{I}(U)\right|
$$

This expression is invariant under modular symplectic transformations in $S p\left(2 h^{(2,1)}+2, \mathbb{Z}\right)$. Note that this type of potential was already discussed in the context of automorphic functions on Calabi-Yau spaces in [123]. Moreover a very similar expression appears as the superpotential due to RR- and NSNS-fluxes on Calabi-Yau spaces, e.g. discussed in 89, 90, 91, 94]. The vacuum structure of this type of potentials was investigated in some detail in [91] with result that at a generic point in the moduli space supersymmetry is completely broken. However the minima of $\mathcal{V}$ are generically such that the system is dynamically driven to those degeneration points in the moduli space (large complex structure limit, conifold points) where full $\mathcal{N}=2$ supersymmetry is restored. In addition, there might by isolated singular points (field theory Seiberg-Witten points), where only $\mathcal{N}=1$ supersymmetry is unbroken. A general analysis of those supersymmetric attractor points was also provided in a beautiful work of Moore 124,125 and also in the context of supersymmetric $\mathcal{N}=2$ black holes in 126,127.

The tree level scalar potential $\mathcal{V}$ was computed in [11,21] (see also [24]) for the case of intersecting D6-branes on $T^{6}$ and also for the case of the $T^{6} / \mathbb{Z}_{3}$ orbifold. For the case of the toroidal background, $\mathcal{V}$ only depends on the three complex structure moduli $U^{I}$ of the three two-tori. For the $\mathbb{Z}_{3}$ orbifold with $h^{(2,1)}=0$, it is simply constant. Let us "check" the general Calabi-Yau formula (8.9) for the case of the torus $T_{1}^{2} \times T_{2}^{2} \times T_{3}^{2}$ with three complex structure moduli $U^{I}, I=1,2,3$. This discussion also applies for every orbifold with $h^{(2,1)}=3$ like the $\mathbb{Z}_{2} \times \mathbb{Z}_{2}$ orbifold. The moduli space for these three fields is $\widetilde{\mathcal{M}}_{\mathrm{H}}=(S U(1,1) / U(1))^{3}$. The corresponding prepotential for the complex structure moduli is given by the expression

$$
F(X)=\frac{X^{1} X^{2} X^{3}}{X^{0}}=\left(X^{0}\right)^{2} U^{1} U^{2} U^{3}, \quad U^{I}=\frac{X^{I}}{X^{0}}
$$


Then, first without any further restriction on the expansion coefficients $e_{I}$ and $m^{I}$, the scalar potential takes the following form

$$
\mathcal{V}=T_{6} e^{-\phi_{4}} \frac{\left|e_{0}+e_{1} U^{1}+e_{2} U^{2}+e_{3} U^{3}+m^{0} U^{1} U^{2} U^{3}+m^{1} U^{2} U^{3}+m^{2} U^{1} U^{3}+m^{3} U^{1} U^{2}\right|}{\prod_{I=1}^{3} \sqrt{\Im\left(U^{I}\right)}}
$$

However, we want to assume that the D6-brane wraps such homology 3-cycles which factorizes into three 1-cycles around each of the three tori; hence this 3-cycle can be characterized by three pairs of unrestricted wrapping numbers $\left(r_{I}, s_{I}\right)$. This means that the $e_{I}$ and $m^{I}$ in (8.11) are not completely arbitrary but the following relations have to hold:

$$
\begin{aligned}
& e_{0}=r_{1} r_{2} r_{3}, \quad e_{1}=s_{1} r_{2} r_{3}, \quad e_{2}=s_{2} r_{1} r_{3}, \quad e_{3}=s_{3} r_{1} r_{2}, \\
& m^{0}=s_{1} s_{2} s_{3}, \quad m^{1}=r_{1} s_{2} s_{3}, \quad m^{2}=r_{2} s_{1} s_{3}, \quad m^{3}=r_{3} s_{1} s_{2} .
\end{aligned}
$$

Then, the potential 8.11) can be rewritten as

$$
\mathcal{V}=T_{6} e^{-\phi_{4}} \prod_{I} \frac{\left|r_{I}+s_{I} U^{I}\right|}{\sqrt{\Im\left(U^{I}\right)}}
$$

This expression agrees with the potential obtained in [11,21] and in [24].

\subsection{Gauge couplings}

Each gauge factor supported on a stack of D6-branes comes with its own gauge coupling $g_{a}$, which can be deduced from the Dirac-Born-Infeld action to be

$$
\frac{1}{g_{a}^{2}}=\frac{M_{s}^{3}}{(2 \pi)^{4}} e^{-\phi_{4}}\left|\int_{\pi_{a}} \widehat{\Omega}_{3}\right|
$$

Thus, the gauge coupling also depends on the volume of the sLag 3-cycle the D6-brane is wrapping around. In a supersymmetric theory the gauge coupling can be combined with an axionic theta-angle into the holomorphic gauge kinetic function $f$. The relevant axionic coupling is contained in the Chern-Simons part of the D6-brane action and looks like

$$
\mathcal{S}_{\text {axion }}=\mu_{6} \int_{\mathbb{R}^{3,1} \times \pi_{a}} 2 \pi^{2}\left(\alpha^{\prime}\right)^{2} C_{3} \wedge \operatorname{Tr}\left(\mathcal{F}_{a} \wedge \mathcal{F}_{a}\right)
$$

Hence, the axion is given by

$$
\lambda_{a}=\frac{2 M_{s}^{3}}{(2 \pi)^{4}} \int_{\pi_{a}} C_{3}
$$


These two couplings combine into a complex coupling

$$
f=\frac{M_{s}^{3}}{(2 \pi)^{4}}\left[e^{-\phi_{4}} \int_{\pi_{a}} \Re\left(\widehat{\Omega}_{3}\right)+2 i \int_{\pi_{a}} C_{3}\right]
$$

where the real part depends on the $h^{(2,1)}$ real complex structure moduli. This was already shown for the toroidal case in [19]. For a general Calabi-Yau, the complex gauge coupling depend holomorphically on the $h^{(2,1)}+1$ chiral superfields that contain the complex structure moduli as scalar components. Moreover, it is known that for $\mathcal{N}=1$ supersymmetric theories the gauge kinetic function receives quantum correction only at the one loop level. Thus, as in 128 one will get corrections from world-sheet instantons with annulus, Möbius strip and Klein-bottle topology. Since the gauge couplings depend on the volume of the 3cycles, it is clear that the constraints one gets for realistic intersecting brane world models to meet the phenomenological gauge couplings at the weak scale are rather moderate and at least at the classical level easy to satisfy by choosing appropriate values for the complex structure moduli.

Finally, we would like to comment on the possibility to obtain scenarios with a low string scale and large extra dimensions 62,63 with intersecting D6-branes. Since the four-dimensional Planck mass is given by $M_{p l}^{2}=M_{s}^{8} e^{-2 \phi_{4}}$, the following relation holds

$$
\frac{1}{g_{a}^{2}} \sim \frac{M_{p l}}{M_{s}}\left|\int_{\pi_{a}} \widehat{\Omega}_{3}\right|
$$

so that gauge couplings of order one can be obtained for small values of the string scale, if we can choose

$$
\left|\int_{\pi_{a}} \widehat{\Omega}_{3}\right| \ll 1
$$

for all 3-cycles $\pi_{a}$. In the toroidal case with factorizable D6-branes it was not possible to meet the requirement (8.19) for all D6-branes while preserving chirality. This was due to the fact, that a large transverse volume requires at least some of the complex structure moduli to become large. Then, (8.18) was violated, because in order to preserve chirality not all D6-branes on a torus could avoid the large direction. This obstruction does no longer appear for general Calabi-Yau models, where low scale string models hence seem to be accessible. 


\section{Mirror symmetry}

So far we have considered type IIA orientifolds with A-type D6-branes wrapping sLag 3-cycles in the Calabi-Yau. We have formulated the consistency conditions for the absence of RR-charges on the internal Calabi-Yau and have presented a general purely topological formula for the chiral massless spectrum localized at the intersection points of the D6branes. In this section we will give the mirror symmetric formulation of such intersecting brane world models.

Here, one starts with the type IIB string on the mirror Calabi-Yau space $\mathcal{W}^{6}$ with Hodge numbers $\widetilde{h}^{(2,1)}=h^{(1,1)}$ and $\widetilde{h}^{(1,1)}=h^{(2,1)}$. Let us briefly discuss the fate of the intersecting D6-branes and the orientifold O6-plane under mirror symmetry. It is known that A-type branes are mapped under mirror symmetry to B-type branes [129], which are wrapped on even-dimensional holomorphic cycles in the $H_{2 p}\left(\mathcal{W}^{6} ; \mathbb{Z}\right)$ homology of the mirror manifold and carry a holomorphic stable gauge bundle with curvature 2 -form $\mathcal{F}$ on their world volumes. The calibration condition $\Re\left(e^{i \theta} \Omega_{3}\right)=d$ vol $\left.\right|_{\mathrm{D} q}$ of the A-branes, is now replaced by the MMMS equation 130

$$
\frac{1}{3 !} \mathcal{F} \wedge \mathcal{F} \wedge \mathcal{F}-\frac{1}{2 !} J \wedge J \wedge \mathcal{F}=\tan (\theta)\left(\frac{1}{2 !} J \wedge \mathcal{F} \wedge \mathcal{F}-\frac{1}{3 !} J \wedge J \wedge J\right)
$$

together with the requirement, that the $(2,0)$ component of $\mathcal{F}$ vanishes. The parameter $\theta$ takes the role of the free phase in the angle condition (2.29) of the sLag cycles. For simplicity, we will take all branes to be D9-branes. The precise map of intersecting D6branes to D9-branes with constant gauge field strength is known for the torus, but for a general Calabi-Yau it is hard to find explicitly. In the case of orbifold realizations mirror symmetry is simply given by T-duality along three of the six compact directions which maps a symmetric $\mathbb{Z}_{N}$ orbifold to an asymmetric $\hat{\mathbb{Z}}_{N}$ orbifold. Moreover, intersecting D6-branes are mapped to D9-branes with constant magnetic flux as has been studied in [1, 73,131$]$.

Even without knowing the precise mirror map for a generic Calabi-Yau model we can independently formulate the models in the mirror symmetric setting. Thus, we start with the type IIB string theory compactified on a Calabi-Yau manifold $\mathcal{W}^{6}$. Now, we divide out this model by the world-sheet parity transformation $\Omega$. As a result we get an orientifold O9-plane wrapping the entire Calabi-Yau manifold and O5-planes wrapping holomorphic 2-cycles $\pi_{\mathrm{O} 5}$ of the Calabi-Yau. In principle, a mirror configuration could also contain O7- and O3-planes, however, not together with O9- and O5-planes, as this would break 
supersymmetry. Now we introduce stacks of $\mathrm{D} 9_{a}$-branes $^{5}$ wrapping the entire threefold $n_{a}$ times and each stack carrying a $U\left(N_{a}\right)$ gauge bundle defined by $\mathcal{F}_{a}$. Via the Chern-Simons couplings (2.8) in the Born-Infeld action these fluxes introduce charges under the lower degree RR-forms as well. Under $\Omega$ a stack of D9-branes with a $U\left(N_{a}\right)$ gauge connection $\mathcal{A}_{a}$ is mapped to another stack of D9-branes with a $U\left(N_{a}\right)$ gauge connection

$$
\mathcal{A}_{a}^{\prime}=-\gamma_{a} \mathcal{A}_{a}^{T} \gamma_{a}^{-1}
$$

where the $\gamma_{a}$ denote unitary matrices. Since the $\mathcal{A}_{a}$ are skew-Hermitian this is identical to

$$
\mathcal{A}_{a}^{\prime}=\gamma_{a} \mathcal{A}_{a}^{*} \gamma_{a}^{-1},
$$

implying that the action on the gauge field strength $\mathcal{F}_{a}=d \mathcal{A}_{a}+\mathcal{A}_{a} \wedge \mathcal{A}_{a}$ is also given by

$$
\mathcal{F}_{a}^{\prime}=\gamma_{a} \mathcal{F}_{a}^{*} \gamma_{a}^{-1}
$$

The RR-tadpole cancellation conditions can be derived from the Chern-Simons couplings

$$
\begin{aligned}
\mathcal{S}_{\mathrm{CS}}=\mu_{9} \sum_{p}\left(\int_{\mathcal{W}^{6}} C_{p} \wedge \sum_{a} n_{a}[\right. & \left.\operatorname{ch}\left(\mathcal{F}_{a}\right)+\operatorname{ch}\left(\mathcal{F}_{a}^{*}\right)\right] \wedge \sqrt{\hat{\mathcal{A}}(\mathcal{R})} \\
+ & \left.Q_{9} \int_{\mathcal{W}^{6}} C_{p} \wedge \sqrt{\hat{\mathcal{L}}(\mathcal{R} / 4)}\right)+\mu_{5} Q_{5} \int_{\pi_{\pi_{5}}} C_{6}
\end{aligned}
$$

and can be written as

$$
\sum_{a} n_{a}\left[\operatorname{ch}\left(\mathcal{F}_{a}\right)+\operatorname{ch}\left(\mathcal{F}_{a}^{*}\right)\right] \wedge \sqrt{\hat{\mathcal{A}}(\mathcal{R})}-32 \sqrt{\hat{\mathcal{L}}(\mathcal{R} / 4)}-2 \frac{\mu_{5}}{\mu_{9}} \delta\left(\pi_{\mathrm{O} 5}\right)=0
$$

where $\delta\left(\pi_{\mathrm{O} 5}\right)$ denotes the Poincaré dual 4 -form of $\pi_{\mathrm{O} 5}$. Since on a Calabi-Yau manifold one has $h^{(0,0)}=h^{(3,3)}=1$ and $h^{(1,1)}=h^{(2,2)}$, expanding the tadpole condition (9.6) into a basis one gets $2 h^{(1,1)}+2$ different RR-forms and their respective tadpole cancellation conditions. However, similar to the type IIA orientifolds only half of these conditions are non-trivial, as one half of the RR-forms are projected out.

In the large volume regime the chiral massless spectrum can be deduced from the Atiyah-Singer index theorem. The four-dimensional chiral fermions transforming in the

5 As in the toroidal case we expect that D-branes of smaller dimension can be considered as special degeneration limits of bundles over D9-branes. The appropriate framework to describe such degenerate bundles is given by coherent sheaves [132. 
bi-fundamental representation of two factors of the total gauge group are sections in a spinor bundle associated to the tensor product of the two individual gauge bundles. Due to the property $\operatorname{ch}\left(\mathcal{F}_{a} \otimes \mathcal{F}_{b}\right)=\operatorname{ch}\left(\mathcal{F}_{a}\right) \wedge \operatorname{ch}\left(\mathcal{F}_{b}\right)$ of the Chern character, their multiplicity is given by

$$
\begin{aligned}
I(a, b) & =\frac{n_{a} n_{b}}{N_{a} N_{b}} \int_{\mathcal{W}^{6}} \operatorname{ch}\left(\bar{N}_{a}, N_{b}\right) \\
& =\frac{n_{a} n_{b}}{N_{a} N_{b}} \int_{\mathcal{W}^{6}} \operatorname{ch}\left(\mathcal{F}_{a}^{*}\right) \wedge \operatorname{ch}\left(\mathcal{F}_{b}\right) \wedge \hat{\mathcal{A}}(\mathcal{R})
\end{aligned}
$$

For the case of flat D9-branes with constant gauge curvatures this formula can also be produced from the open string quantization, of course. Moreover, the number of $\Omega$ invariant self-intersection points is given by the Möbius amplitude, which was derived in [133],

$$
I(a)=8 \frac{n_{a}}{N_{a}} \int_{\mathcal{W}^{6}} \operatorname{ch}\left(\mathcal{F}_{a}\right) \wedge \sqrt{\hat{\mathcal{A}}(\mathcal{R})} \wedge \sqrt{\hat{\mathcal{L}}(\mathcal{R} / 4)}+\frac{1}{2} \frac{n_{a}}{N_{a}} \int_{\pi_{\mathrm{O} 5}} \operatorname{ch}\left(\mathcal{F}_{a}\right)
$$

Thus, the spectrum of chiral fermions for a set of D9-branes with gauge fluxes is now given by table 15 .

\begin{tabular}{|c|c|}
\hline Representation & Multiplicity \\
\hline$\left[\mathbf{A}_{\mathbf{a}}\right]_{L}$ & $\frac{1}{2}\left(I\left(a^{*}, a\right)+I(a)\right)$ \\
\hline$\left[\mathbf{S}_{\mathbf{a}}\right]_{L}$ & $\frac{1}{2}\left(I\left(a^{*}, a\right)-I(a)\right)$ \\
\hline$\left[\left(\overline{\mathbf{N}}_{\mathbf{a}}, \mathbf{N}_{\mathbf{b}}\right)\right]_{L}$ & $I(a, b)$ \\
\hline$\left[\left(\mathbf{N}_{\mathbf{a}}, \mathbf{N}_{\mathbf{b}}\right)\right]_{L}$ & $I\left(a^{*}, b\right)$ \\
\hline
\end{tabular}

Table 15: $\quad$ Chiral spectrum in $d=4$

Using the RR-tadpole cancellation condition (9.6) it is straightforward to confirm that the generic massless spectrum in table 15 is free of non-abelian gauge anomalies.

As a simple check one can verify that the Atiyah-Singer index theorem (9.7) applied to $U(1)$ gauge bundles with constant field strength on a flat six-torus $T^{6}=T_{1}^{2} \times T_{2}^{2} \times T_{3}^{2}$ indeed gives the result derived in [1]. The following constant $U(1)$ gauge fluxes are T-dual to factorizing intersecting D6-branes on $T^{6}$

$$
\mathcal{F}_{a}=-2 \pi i \sum_{I=1}^{3} \frac{m_{a}^{I}}{n_{a}^{I} \operatorname{Vol}\left(T_{I}^{2}\right)} \mathbf{1}_{\left(N_{a}, N_{a}\right)} d x_{I} \wedge d y_{I}
$$


where $x_{I}, y_{I}$ are Cartesian coordinates on the torus $T_{I}^{2}$. The evaluation of the integral in (9.7) for the number of chiral fermions

$$
I(a, b)=\prod_{I=1}^{3}\left(n_{a}^{I} m_{b}^{I}-m_{a}^{I} n_{b}^{I}\right)
$$

which agrees with the formula deduced in [1] by CFT methods. One can push this list of analogies further, for instance in evaluating the potential in the dual frame. In the end, the two mirror sides are just equivalent descriptions of identical physics.

\section{Lift to M-theory and related issues}

In the previous sections we have described in quite general terms intersecting D6-brane models on general orientifolds of Calabi-Yau manifolds. These models provide a huge class of four-dimensional string compactifications with quasi-realistic gauge groups and chiral matter content, where the gauge degrees of freedom are localized on the D6-branes with co-dimension three and the chiral matter sits at the intersections of these D6-branes of co-dimension six. In general, such models break supersymmetry completely, but this is not automatic. Demanding supersymmetry actually fixes some of the complex structure moduli dynamically and deviating from the supersymmetric locus introduces a D-term potential, such that the breaking can be described spontaneously. In order to have any chance to preserve supersymmetry in these models, it is essential that we are dealing with orientifolds containing O6-planes with negative tension, so that the overall tension can potentially vanish.

An interesting issue in type IIA vacua with only D6-branes, and possibly O6-planes, is the fact that the eleven-dimensional lift to M-theory is purely geometrical in the sense that the eleven-dimensional 3 -form is trivial. This can be easily seen by looking at the fields which couple to the 6-brane background. The D6-branes are the magnetically charged monopoles of the KK vector, and thus only couple to components of the eleven-dimensional metric, the RR 1-form $C_{1}$ and the dilaton of type IIA. The geometric lift of isolated D6-branes in flat ten-dimensional space-time is then a non-trivial $U(1)$ fibration over an $S^{2} \subset \mathbb{R}^{3}$, a Taub-Nut space. The base $S^{2}$ is given by the asymptotic infinity of their transverse space $\mathbb{R}^{3}$ in ten-dimensions, and the $U(1)$ fiber defines the dilaton of type IIA. Similarly, the O6-planes lift to an Atiyah-Hitchin space. It is important that these spaces are equipped with metrics that allow a consistent ten-dimensional interpretation of 
the configuration. This requires in particular, that the fiber circle is regular everywhere and approaches a finite radius at infinity. Then, the geometry corresponds to a type IIA vacuum with a finite coupling everywhere, which upon rescaling may be assumed to be small at infinity.

In order to get a chiral model it is not sufficient to consider parallel D6-branes and O6-planes, as we have discussed at length. In chiral backgrounds the charges cannot cancel locally and the dilaton and the RR 1-form potential vary. Supersymmetric settings of this type preserve exactly four supercharges, i.e. $\mathcal{N}=1$ supersymmetry in four dimensions. By the general classification of the number of Killing spinors on a seven-dimensional manifold equipped with the Levi-Civita connection, they lift to an M-theory compactification on a manifold with holonomy $G_{2}$. Due to the fact, that smooth $G_{2}$-manifolds only have abelian isometries, to lift a chiral model with a non-abelian gauge group, we need to allow for singularities [134, 135, [3, [36]. In practice, little is known about the lift of compact six-dimensional backgrounds to eleven dimensions, except for orbifold models [137 and CFT results [138,139,140,141,142], which, however, do not directly relate to a geometric interpretation of the manifold. In the following section we just like to collect a number of observations and speculations starting from the known cases of local models. In a sense, we would like to take the attitude that supersymmetric orientifolds with intersecting branes in ten dimensions provide an alternative approach to the study of M-theory compactifications on certain singular $G_{2}$ manifolds. ${ }^{6}$

\subsection{Local models of intersecting D6-branes}

It has been argued, that the proper lift of two intersecting D6-banes in $\mathbb{R}^{6}$ is given by a non-compact seven-dimensional cone [149]. Concretely, it was observed by M. Atiyah and E. Witten that one can define a $U(1)$ action on $\mathbb{C P}^{3}$ such that $\mathbb{C P}^{3} / U(1)=S^{5}$ and $\operatorname{Fix}(U(1))=S^{2} \cup S^{2}$. Taking the seven-dimensional cone on $\mathbb{C P}^{3}$ leads to a $U(1)$-manifold with a fiber that degenerates exactly along two intersecting sLag 3-planes within $\mathbb{R}^{6}$. Smoothing out this geometry is believed to describe the geometric lift of two intersecting D6-branes located at the fixed locus of the $U(1)$ that defines the dilaton. Actually, a

6 It would be also interesting to get a better understanding about the relation between Mtheory on a compact $G_{2}$ manifold respectively intersecting D6-branes in type IIA and orbifold compactifications of heterotic M-theory to six and four dimensions with chiral matter fields located at the intersections of the various orbifold fixed planes 143-148]. 
metric on the cone on $\mathbb{C P}^{3}$ with holonomy $G_{2}$ is known [150,151,152]. It describes a space topologically $\mathbb{R}^{3} \times S^{4}$, where the co-associative 4-cycle $S^{4}$ replaces the singular tip of the cone. Unfortunately, this metric can not function as a candidate for the lift of the type IIA vacuum without modifications, as the circle meant to define the string coupling is not finite at infinity.

In 153 (see also [154]) a generalization of the construction towards multiple inter-

secting D6-branes was proposed. Using $\mathbb{C P}^{3} / U(1)=S^{7} / U(1)^{2}$, one can divide out by any linear combination $U(1)^{\prime}$ of the two $U(1)$ in the first step to define the base of another seven-dimensional cone. The linear combination of the two generators is defined by two integers $(n, m)$, which simultaneously play the role of the weights of the weighted projective space $S^{7} / U(1)^{\prime}=\mathbf{W C P}_{n, n, m, m}^{3}$ and of the multiplicities of the two D6-branes. These are now modeled by the $A_{n-1}$ and $A_{m-1}$ singularities of the weighted projective space. Not even knowing the proper metric to describe the lift of a single pair of D6-branes, there appear to be little clues, how to determine a sufficiently regular metric for this more demanding case (see e.g. [155] for some discussion). Still, one may want to add more physical observations about what we expect these spaces to behave like, even without knowing their precise definition.

\subsection{Geometric transitions}

In particular, in the type IIA background we encounter a number of transitions which should lift to viable topology changing transitions of the $G_{2}$ manifold. At a supersymmetric intersection of two D6-branes the bosonic superpartner of the chiral fermion is a marginal operator transforming in the bi-fundamental representation of the two gauge factors. This flat direction indicates a deformation of the two intersecting D6-branes towards a single D6-brane with the same volume while preserving the homology class [156]. This transition can be thought of as the six-dimensional analogue of the deformation of sLag 2-cycles, that was studied in section 4.7.1. In the effective field theory it can be considered as moving on a Higgs-branch, where the gauge symmetry of the two individual stacks is broken to the diagonal. It is clear that in the M-theory such a smooth transition must lift to a topology change between the two different singular $G_{2}$ manifolds described above. In a local model, we then expect a smooth transition between seven-dimensional cones on different threedimensional weighted projective spaces. 
An even more dramatic change is given by the conifold transition, where a 3-cycle in the Calabi-Yau shrinks to zero size and the resulting singularity is resolved by a 2cycle. Following C. Vafa [157] D6-branes which are wrapped around the shrinking 3-cycle become some RR 2-form flux through the resolving 2-sphere. In fact, this type of topology change is quite well understood for the deformed conifold geometry $T^{*} S^{3}$ with $N$ wrapped D6-branes on the sLag $S^{3}$, and the resolved conifold $\mathcal{O}(-1) \oplus \mathcal{O}(-1) \rightarrow \mathbb{C P}^{1}$ with $N$ units of flux for the RR 2-form $G_{2}$. In the corresponding M-theory lift, this situation was geometrically interpreted by a flop transition between two $S^{3}$ in the corresponding noncompact $G_{2}$ manifold [158]. Here, the situation is slightly better in that an appropriate metric on one side of the transition is known, while for the metric after the flop numerical studies have been performed [159,160.

For compact Calabi-Yau manifolds the conifold transition affects the whole Calabi-Yau manifold. Then, in general we have to expect that after the transition all the D6-branes and the O6-planes are arranged in a completely new way. Whether this quite complicated type IIA transition lifts to a simple flop transition on the $G_{2}$ manifold is clearly a difficult question. But one may try to derive at least some consistency requirement from the tadpole conditions analogous to (2.14). So let us assume that we are left after the conifold transition with some remaining 3-cycles $\pi_{a}\left(a=1, \ldots, N^{\prime}\right)$ with $N_{a}$ D6-branes wrapped around as well as with the $\mathrm{O} 6$ homology 3 -cycle $\pi_{\mathrm{O} 6}$. In addition $N^{\prime \prime}$ new 2-cycles $S_{b}^{2}$ $\left(b=1, \ldots, N^{\prime \prime}\right)$ have emerged after the conifold transition, which each supports $N_{b}$ units of Ramond 2-form flux:

$$
N_{b}=\int_{S_{b}^{2}} G_{2}, \quad G_{2}=d C_{1} .
$$

Before we try to generalize the type IIA tadpole condition including the Ramond 2 -form fluxes, let us switch to the type IIB mirror picture. Here the conifold transition replaces D5-branes wrapped around a 2-sphere by some flux of the 3-form gauge field strength $H_{3}^{\mathrm{R}}+\tau H_{3}^{\mathrm{NS}}$ through some appropriate dual 3-cycles $C_{b}^{3}$ and $\tilde{C}_{b}^{3}$. Then we will get the following tadpole condition for the 3 -form fluxes in the absence of space-time filling D3-branes (see e.g. the discussion in [97]):

$$
\sum_{b=1}^{N^{\prime \prime}} \int_{C_{b}^{3} \times \tilde{C}_{b}^{3}} H_{3}^{\mathrm{R}} \wedge H_{3}^{\mathrm{NS}}=0 .
$$

Coming back to the type IIA side, it was argued in [157] (see also the comments in [161]) that the mirror description of the type IIB NS 3-form flux is given in type IIA in 
terms of a non-vanishing flux of a NS 4-form field strength $H_{4}^{\mathrm{NS}}$. Actually the role of this NS 4-form field strength is still not very well understood in the literature. It is associated with the lack of integrability of the complex structure of the Calabi-Yau space due to the presence of the Ramond 2-form flux. Hence $H_{4}^{\mathrm{NS}}$ reflects the back-reaction of turning on the Ramond 2-form flux on the six-dimensional Calabi-Yau metric. In any case, if this interpretation is correct, the following tadpole condition for the Ramond 2-form fluxes suggests itself:

$$
\sum_{b=1}^{N^{\prime \prime}} \int_{S_{b}^{2} \times C_{b}^{4}} G_{2} \wedge H_{4}^{\mathrm{NS}}=0 .
$$

Here the sum is meant to be over the relevant homology 2-cycles $S_{b}^{2}$. In addition we have also to satisfy the tadpole condition for the still remaining wrapped D6-branes:

$$
\sum_{a}^{N^{\prime}} N_{a}\left(\pi_{a}+\pi_{a}^{\prime}\right)=4 \pi_{O 6} .
$$

These two equations constitute two sets of independent conditions, one for the wrapped D6-branes on the homology 3-cycles, and a second for the fluxes through the homology 2-cycles. Therefore due to these two equations we expect that the conifold transition with D6-branes on a compact CY-space is only possible under strong restrictions, namely only if the disappearing D6-branes, and hence at the same the newly emerging 2-form fluxes satisfy the Ramond tadpole conditions on their own.

\section{Conclusions}

In this paper we have investigated aspects of intersecting brane worlds on orientifolds of K3 and Calabi-Yau manifolds. As our main result we have provided a general formula for the chiral massless spectrum in terms of topological invariants. In view of the AtiyahSinger index theorem it is required that the chiral fermions are determined entirely by the topology of the configurations when classical geometry is valid. However, after having witnessed the construction of various orientifold models over the years using explicit and elaborate conformal field theory techniques, it appears to be quite amusing that the most important part of the massless spectra can be so easily determined from the intersection numbers of cycles in the blown-up geometries. As a new example, which mainly just demonstrates the power of the general topological formulas, we derived a "realistic" three 
generation Standard-like Model from wrapping D-branes on 3-cycles of the quintic CalabiYau.

We have pointed out that these intersecting brane world models are interesting for many reasons. From the phenomenological point of view they generically produce effective six- and four-dimensional models with chiral fermions and do provide an intrinsic mechanism to break supersymmetry. In order for realistic non-supersymmetric models to make sense it is viable that the string scale is not much large than the TeV scale. It would be interesting to construct concrete models for which such a low string scale scenario is realized. But the central issue in non-supersymmetric model building still remains to be the problem of stabilizing the scalar fields, in the first place the dilaton, of course.

For specific choices of the intersecting branes it is possible to get models with $\mathcal{N}=1$ supersymmetry in four dimensions. For these models it would be a major step forward if one could determine the instanton generated F-term superpotential using mirror symmetry. In particular, it would be interesting to see in how far there exist intersecting brane worlds which are not destabilized by such potentials. At least we know that supersymmetric intersecting brane worlds on orbifold backgrounds are exact minima of the scalar potential. Of course, the issue of supersymmetry breaking is crucial to relate these models to the low-energy world. Moreover, every supersymmetric intersecting brane world is expected to lift to an M-theory compactification on a singular compact $G_{2}$ manifold. Since not very much is known about such $G_{2}$ manifolds, intersecting branes might provide an important approach to detect at least part of their structure.

Another important problem in four-dimensional intersecting brane world models is the question of gauge coupling unification, or respectively, to obtain realistic values for the gauge couplings. This seems to be quite non-trivial in non-supersymmetric models with a TeV string scale, but probably easier in supersymmetric models with a high string scale and low-energy supersymmetry breaking. In any case, to analyze this problem one has to compute the higher loop threshold corrections to the gauge couplings of the open strings on the Calabi-Yau manifolds.

Finally, let us mention the possibility of spontaneously breaking the gauge group of the Standard Model by a tachyonic scalar field which has the quantum numbers of the Standard Model Higgs field. This mechanism was discussed in [1, 6, 8, 11, 22, and it is described in terms of brane language by the recombination of the $S U(2)_{L}$ and $U(1)_{Y}$ D-branes into a single $U(1)_{Q}$ D-brane. Following the change of intersecting numbers triggered by the D-brane recombination process, information about the mass generation 
for quarks and leptons can be in principle obtained. Therefore, a careful examination of the tachyon potential and the rearrangement of topological D-brane intersection numbers due to tachyon condensation on Calabi-Yau spaces would be worth to be investigated. In summary, a lot of theoretical and phenomenological problems related to D-branes on Calabi-Yau spaces are still to be solved.

\section{Acknowledgments}

We would like to thank A. Klemm for useful discussions. The work is supported in part by the EC under the RTN project HPRN-CT-2000-00131. R.B. would like to thank the Theoretical Physics groups at the University Bonn and at the Universidad Autonoma de Madrid in addition to ISAS-SISSA Trieste for hospitality. We thank L. Görlich and T. Ott for pointing out an error in an earlier version of this paper.

R.B. would like to dedicate this paper to the memory of his colleague Sonia Stanciu. He shared a creative time with her as graduate students at the University Bonn in the early nineties. 


\section{References}

[1] R. Blumenhagen, L. Görlich, B. Körs and D. Lüst, Noncommutative Compactifications of Type I Strings on Tori with Magnetic Background Flux, JHEP 0010 (2000) 006, hep-th/0007024.

[2] C. Angelantonj, I. Antoniadis, E. Dudas, A. Sagnotti, Type I Strings on Magnetized Orbifolds and Brane Transmutation, Phys. Lett. B 489 (2000) 223, hep-th/0007090.

[3] R. Blumenhagen, L. Görlich, B. Körs and D. Lüst, Magnetic Flux in Toroidal Type I Compactification, Fortsch. Phys. 49 (2001) 591, hep-th/0010198.

[4] C. Angelantonj, A. Sagnotti, Type I Vacua and Brane Transmutation, hep-th/0010279.

[5] G. Aldazabal, S. Franco, L. E. Ibanez, R. Rabadan, A. M. Uranga, D = 4 Chiral String Compactifications from Intersecting Branes, J. Math. Phys. 42 (2001) 3103, hep-th/0011073.

[6] G. Aldazabal, S. Franco, L. E. Ibanez, R. Rabadan, A. M. Uranga, Intersecting Brane Worlds, JHEP 0102 (2001) 047, hep-ph/0011132.

[7] R. Blumenhagen, B. Körs and D. Lüst, Type I Strings with $F$ and B-Flux, JHEP 0102 (2001) 030, hep-th/0012156.

[8] L. E. Ibanez, F. Marchesano, R. Rabadan, Getting just the Standard Model at Intersecting Branes, JHEP 0111 (2001) 002, hep-th/0105155.

[9] S. Förste, G. Honecker and R. Schreyer, Orientifolds with Branes at Angles, JHEP 0106 (2001) 004, hep-th/0105208.

[10] R. Rabadan, Branes at Angles, Torons, Stability and Supersymmetry, Nucl. Phys. B 620 (2002) 152, hep-th/0107036.

[11] R. Blumenhagen, B. Körs, D. Lüst and T. Ott, The Standard Model from Stable Intersecting Brane World Orbifolds, Nucl. Phys. B 616 (2001) 3, hep-th/0107138.

[12] M. Cvetic, G. Shiu and A. M. Uranga, Three-Family Supersymmetric Standard-like Models from Intersecting Brane Worlds Phys. Rev. Lett. 87 (2001) 201801, hepth/0107143.

[13] M. Cvetic, G. Shiu and A. M. Uranga, Chiral Four-Dimensional N=1 Supersymmetric Type IIA Orientifolds from Intersecting D6-Branes, Nucl. Phys. B 615 (2001) 3, hepth/0107166.

[14] D. Bailin, G. V. Kraniotis and A. Love, Standard-like Models from Intersecting D4branes, Phys. Lett. B 530 (2002) 202, hep-th/0108131.

[15] L. E. Ibanez, Standard Model Engineering with Intersecting Branes, hep-ph/0109082.

[16] R. Blumenhagen, B. Körs, D. Lüst and T. Ott, Intersecting Brane Worlds on Tori and Orbifolds, hep-th/0112015.

[17] G. Honecker, Non-supersymmetric Orientifolds with D-branes at Angles, hep-th/0112174.

[18] G. Honecker, Intersecting Brane World Models from D8-branes on $\left(T^{2} \times T^{4} / \mathbb{Z}_{3}\right) / \Omega R_{1}$ Type IIA Orientifolds, JHEP 0201 (2002) 025, hep-th/0201037. 
[19] D. Cremades, L. E. Ibanez and F. Marchesano, SUSY Quivers, Intersecting Branes and the Modest Hierarchy Problem, hep-th/0201205.

[20] R. Blumenhagen, B. Körs and D. Lüst, Moduli Stabilization for Intersecting Brane Worlds in Type 0'String Theory, hep-th/0202024.

[21] R. Blumenhagen, B. Körs, D. Lüst and T. Ott, Hybrid Inflation in Intersecting Brane Worlds, hep-th/0202124.

[22] D. Cremades, L. E. Ibanez and F. Marchesano, Intersecting Brane Models of Particle Physics and the Higgs Mechanism, hep-th/0203160.

[23] C. Kokorelis, GUT Model Hierarchies from Intersecting Branes, hep-th/0203187.

[24] J. Garcia-Bellido and R. Rabadan, Complex Structure Moduli Stability in Toroidal Compactifications, JHEP 0205 (2002) 042, hep-th/0203247.

[25] D. Cremades, L. E. Ibanez and F. Marchesano, Standard Model at Intersecting D5branes: Lowering the String Scale, hep-th/0205074.

[26] C. Kokorelis, New Standard Model Vacua from Intersecting Branes, hep-th/0205147.

[27] G. Aldazabal, L. E. Ibanez and A. M. Uranga, Gauging Away the Strong CP Problem, hep-ph/0205250.

[28] M. Cvetic, P. Langacker, and G. Shiu, Phenomenology of A Three-Family Standardlike String Model, hep-ph/0205252.

[29] M. Klein, Couplings in Pseudo-Supersymmetry, hep-th/0205300.

[30] G. Aldazabal and A.M. Uranga, Tachyon-free Non-supersymmetric Type IIB Orientifolds via Brane-Antibrane Systems, JHEP 9910 (1999) 024, hep-th/9908072.

[31] I. Brunner, M. R. Douglas, A. Lawrence and C. Römelsberger, D-branes on the Quintic, JHEP 0008 (2000) 015, hep-th/9906200.

[32] P. Kaste, W. Lerche, C. A. Lutken and J. Walcher, D-branes on K3-fibrations, Nucl. Phys. B 582 (2000) 203, hep-th/9912147.

[33] S. Kachru, S. Katz, A. Lawrence and J. McGreevy, Open String Instantons and Superpotentials, Phys.Rev. D62 (2000) 026001, hep-th/9912151.

[34] K. Hori, A. Iqbal and C. Vafa, D-Branes And Mirror Symmetry, hep-th/0005247.

[35] S. Kachru, S. Katz, A. Lawrence and J. McGreevy, Mirror Symmetry for Open Strings, hep-th/0006047.

[36] W. Lerche, C. A. Lutken and C. Schweigert, D-branes on ALE Spaces and the ADE Classification of Conformal Field Theories, Nucl. Phys. B 622 (2002) 269, hepth/0006247.

[37] M. Aganagic and C. Vafa, Mirror Symmetry, D-Branes and Counting Holomorphic Discs, hep-th/0012041.

[38] M. Aganagic, A. Klemm and C. Vafa, Disk Instantons, Mirror Symmetry and the Duality Web, Z.Naturforsch. A57 (2002) 1-28, hep-th/0105045.

[39] P. Mayr, N=1 Mirror Symmetry and Open/Closed String Duality, hep-th/0108229. 
[40] S. Govindarajan, T. Jayaraman and T. Sarkar, Disc Instantons in Linear Sigma Models, hep-th/0108234.

[41] S. Hellermann, S. Kachru, A. Lawrence and J. McGreevy, Linear Sigma Models for Open Strings, hep-th/0109069.

[42] A. Iqbal, A. K. Kashani-Poor, Discrete Symmetries of the Superpotential and Calculation of Disk Invariants, hep-th/0109214.

[43] W. Lerche and P. Mayr, On N=1 Mirror Symmetry for Open Type II Strings, hepth/0111113.

[44] J. D. Blum, Calculation of Nonperturbative Terms in Open String Models, hepth/0112039.

[45] B. Acharya, M. Aganagic, K. Hori and C. Vafa, Orientifolds, Mirror Symmetry and Superpotentials, hep-th/0202208.

[46] M. Berkooz, M. R. Douglas and R. G. Leigh, Branes Intersecting at Angles, Nucl. Phys. B 480 (1996) 265, hep-th/9606139.

[47] C. Angelantonj and A. Sagnotti, Open Strings, hep-th/0204089.

[48] E. G. Gimon and J. Polchinski, Consistency Conditions for Orientifolds and DManifolds, Phys. Rev. D54 (1996) 1667, hep-th/9601038.

[49] M. Bianchi and A. Sagnotti, On the Systematics of Open String Theories, Phys. Lett. B 247 (1990) 517.

[50] E. G. Gimon and C. V. Johnson, K3 Orientifolds, Nucl. Phys. B 477 (1996) 715, hep-th/9604129.

[51] R. Blumenhagen, L. Görlich and B. Körs, Supersymmetric Orientifolds in 6D with D-Branes at Angles, Nucl. Phys. B 569 (2000) 209, hep-th/9908130.

[52] C. Angelantonj, R. Blumenhagen, Discrete Deformations in Type I Vacua, Phys. Lett. B 473 (2000) 86, hep-th/9911190.

[53] G. Pradisi, Type I Vacua from Diagonal $\mathbb{Z}_{3}$-Orbifolds, Nucl. Phys. B 575 (2000) 134, hep-th/9912218.

[54] R. Blumenhagen, L. Görlich and B. Körs, A New Class of Supersymmetric Orientifolds with D-Branes at Angles, hep-th/0002146.

[55] M. R. Douglas, Branes within Branes, hep-th/9512077.

[56] M. Green, J. A. Harvey, G. Moore, I-Brane Inflow and Anomalous Couplings on DBranes Class. Quant. Grav. 14 (1997) 47, hep-th/9605033.

[57] J. F. Morales, C. A. Scrucca and M. Serone, Anomalous Couplings for D-branes and O-planes, Nucl. Phys. B 552 (1999) 291, hep-th/9812071.

[58] C. A. Scrucca and M. Serone, Anomalies and Inflow on D-branes and O-planes, Nucl. Phys. B 556 (1999) 197, hep-th/9903145.

[59] B. Stefański, jr, Gravitational Couplings of D-branes and O-planes, Nucl. Phys. B 548 (1999) 275, hep-th/9812088. 
[60] E. S. Fradkin and A. A. Tseytlin, Nonlinear Electrodynamics From Quantized Strings, Phys. Lett. B 163 (1985) 123.

[61] R. G. Leigh, Dirac-Born-Infeld Action From Dirichlet Sigma Model, Mod. Phys. Lett. A 4 (1989) 2767.

[62] N. Arkani-Hamed, S. Dimopoulos, and G. Dvali, The Hierarchy Problem and New Dimensions at a Millimeter, Phys. Lett. B 429 (1998) 263, hep-ph/9803315.

[63] I. Antoniadis, N. Arkani-Hamed, S. Dimopoulos, and G. Dvali, New Dimensions at a Millimeter to a Fermi and Superstrings at a TeV, Phys. Lett. B 436 (1998) 257, hep-ph/9804398.

[64] L. J. Dixon, J. A. Harvey, C. Vafa and E. Witten, Strings On Orbifolds, Nucl. Phys. B 261 (1985) 678.

[65] L. J. Dixon, J. A. Harvey, C. Vafa and E. Witten, Strings On Orbifolds 2, Nucl. Phys. B 274 (1986) 285.

[66] J. D. Blum and A. Zaffaroni, An Orientifold from F Theory, Phys.Lett. B 387 (1996) 71, hep-th/9607019.

[67] A. Dabholkar and J. Park, A Note on Orientifolds and F-theory, Phys. Lett. B 394 (1997) 302, hep-th/9607041.

[68] J. D. Blum, F Theory Orientifolds, $M$ Theory Orientifolds and Twisted Strings, Nucl.Phys. B 486 (1997) 34, hep-th/9608053.

[69] A. Dabholkar and J. Park, An Orientifold of Type IIB theory on K3, Nucl. Phys. B 472 (1996) 207, hep-th/9602030; Strings on Orientifolds, Nucl. Phys. B 477 (1996) 701, hep-th/9604178.

[70] A. Sen, A Non-perturbative Description of the Gimon-Polchinski Orientifold, Nucl. Phys. B 489 (1997) 139, hep-th/9611186.

[71] A. Sen, F-theory and the Gimon-Polchinski Orientifold, Nucl. Phys. B498 (1997) 135, hep-th/9702061.

[72] S. Kachru and J. McGreevy, Supersymmetric Three-cycles and (Super)symmetry Breaking, Phys.Rev. D61 (2000) 026001, hep-th/9908135.

[73] R. Blumenhagen, L. Görlich, B. Körs and D. Lüst, Asymmetric Orbifolds, Noncommutative Geometry and Type I Vacua, Nucl. Phys. B 582 (2000) 44, hep-th/0003024.

[74] D. R. Morrison and C. Vafa, Compactifications of F-Theory on Calabi-Yau Threefolds - II, Nucl. Phys. B 476 (1996) 437, hep-th/9603161.

[75] R. L. Bryant, Some Examples of Special Lagrangian Tori, math.DG/9902076

[76] C. Vafa, Evidence for F-Theory, Nucl. Phys. B 469 (1996) 403, hep-th/9602022.

[77] D. R. Morrison and C. Vafa, Compactifications of F-Theory on Calabi-Yau Threefolds - I, Nucl. Phys. B 473 (1996) 74, hep-th/9602114.

[78] A. Sen, F-theory and Orientifolds, Nucl. Phys. B 475 (1996) 562, hep-th/9605150.

[79] T. Banks, M. R. Douglas and N. Seiberg, Probing F-theory with Branes, Phys. Lett. B 387 (1996) 278, hep-th/9605199. 
[80] C. Voisin, Journées de Géométrie Algébrique d'Orsay, eds. A. Beauville et al. (Orsay, 1992), Astérisque No. 218 (1993) 273.

[81] C. Borcea, K3 surfaces with involution and mirror pairs of Calabi-Yau manifolds, in: Essays on Mirror Manifolds II, eds. B. Greene and S.-T. Yau, (International Press and AMS 1997).

[82] V. Nikulin, in: Proceedings of the International Congress of Mathematicians (Berkeley 1986) 654.

[83] D. Joyce, Compact Manifolds of Special Holonomy, Oxford University Press, 2000

[84] S. Kachru and J. McGreevy, M-theory on Manifolds of $G_{2}$ Holonomy and Type IIA Orientifolds, JHEP 0106 (2001) 027, hep-th/0103223.

[85] A. Recknagel and V. Schomerus, D-branes in Gepner models, Nucl. Phys. B 531 (1998) 185, hep-th/9712186.

[86] B. de Wit, D. J. Smit and N. D. Hari Dass, Residual Supersymmetry Of Compactified $D=10$ Supergravity, Nucl. Phys. B 283 (1987) 165.

[87] J. Polchinski and A. Strominger, New Vacua for Type II String Theory, Phys. Lett. B 388 (1996) 736, hep-th/9510227.

[88] J. Michelson, Compactifications of Type IIB Strings to Four Dimensions with nontrivial Classical Potential, Nucl. Phys. B 495, 127 (1997) hep-th/9610151.

[89] T. R. Taylor and C. Vafa, RR flux on Calabi-Yau and Partial Supersymmetry Breaking, Phys. Lett. B 474 (2000) 130, hep-th/9912152.

[90] P. Mayr, On Supersymmetry Breaking in String Theory and its Realization in Brane Worlds, Nucl. Phys. B 593 (2001) 99, hep-th/0003198.

[91] G. Curio, A. Klemm, D. Lüst and S. Theisen, On the Vacuum Structure of Type II String Compactifications on Calabi-Yau Spaces with H-fluxes, Nucl. Phys. B 609 (2001) 3, hep-th/0012213.

[92] M. Haack and J. Louis, M-theory Compactified on Calabi-Yau Fourfolds with Background Flux, Phys. Lett. B 507 (2001) 296, hep-th/0103068.

[93] S. B. Giddings, S. Kachru and J. Polchinski, Hierarchies from Fluxes in String Compactifications, hep-th/0105097.

[94] G. Curio, A. Klemm, B. Körs and D. Lüst, Fluxes in Heterotic and Type II String Compactifications, Nucl. Phys. B 620 (2002) 237, hep-th/0106155.

[95] G. Dall'Agata, Type IIB Supergravity Compactified on a Calabi-Yau Manifold with H-fluxes, JHEP 0111 (2001) 005, hep-th/0107264.

[96] J. Louis and A. Micu, Heterotic String Theory with Background Fluxes, Nucl. Phys. B 626, 26 (2002) hep-th/0110187.

[97] S. Kachru, M. Schulz and S. Trivedi, Moduli Stabilization from Fluxes in a Simple IIB Orientifold, hep-th/0201028.

[98] A. M. Uranga, D-brane, Fluxes and Chirality, hep-th/0201221. 
[99] J. Louis and A. Micu, Type II Theories Compactified on Calabi-Yau Threefolds in the Presence of Background Fluxes, hep-th/0202168.

[100] K. Dasgupta, K. Oh, J. Park and R. Tatar, Geometric Transition versus Cascading Solution, JHEP 0201 (2002) 031, hep-th/0110050.

[101] K. Becker, M. Becker, M. Haack and J. Louis, Supersymmetry Breaking and alpha' Corrections to Flux induced Potentials, hep-th/0204254.

[102] S. Fredenhagen and V. Schomerus, Branes on Group Manifolds, Gluon Condensates, and twisted K-theory, JHEP 0104 (2001) 007, hep-th/0012164.

[103] J. Maldacena, G. Moore, and N. Seiberg, D-Brane Instantons and K-Theory Charges, JHEP 0111 (2001) 062, hep-th/0108100.

[104] M. Berkooz and R. G. Leigh, $A D=4 N=1$ Orbifold of Type I Strings, Nucl. Phys. B 483 (1997) 187, hep-th/9605049.

[105] C. Angelantonj, M. Bianchi, G. Pradisi, A. Sagnotti and Y. Stanev, Chiral Asymmetry in Four-Dimensional Open-String Vacua, Phys.Lett. B 385 (1996) 96, hepth/9606169.

[106] G. Aldazabal, A. Font, L. E. Ibanez and G. Violero, $D=4, N=1$, Type IIB Orientifolds, Nucl. Phys. B 536 (1998) 29, hep-th/9804026.

[107] Z. Kakushadze, On Four-dimensional $N=1$ Type I Compactifications, Nucl. Phys. B 535 (1998) 311, hep-th/9806008.

[108] R. Blumenhagen, L. Görlich and B. Körs, Supersymmetric 4 D Orientifolds of Type IIA with D6-branes at Angles, JHEP 0001 (2000) 040, hep-th/9912204.

[109] S. Förste, G. Honecker and R. Schreyer, Supersymmetric $\mathbb{Z}_{N} \times \mathbb{Z}_{M}$ Orientifolds in 4-D with D-branes at Angles, Nucl. Phys. B 593 (2001) 127, hep-th/0008250.

[110] S. Förste, G. Honecker and R. Schreyer, Orientifolds with Branes at Angles, JHEP 0106 (2001) 004, hep-th/0105208.

[111] C. Bachas, A Way to Break Supersymmetry, hep-th/9503030.

[112] B. Acharya, M. Aganagic, K. Hori and C. Vafa, Orientifolds, Mirror Symmetry and Superpotentials, hep-th/0202208.

[113] H. Ooguri and C. Vafa, Knot Invariants and Topological Strings, Nucl. Phys. B 577 (2000) 419, hep-th/9912123.

[114] M. Aganagic and C. Vafa, Mirror Symmetry, D-branes and Counting Holomorphic Discs hep-th/0012041.

[115] M. Aganagic, A. Klemm and C. Vafa, Disk Instantons, Mirror Symmetry and the Duality Web, Z. Naturforsch. A 57 (2002) 1, hep-th/0105045.

[116] J. Distler, S. Kachru, (0,2) Landau-Ginzburg Theory, Nucl. Phys. B 413 (1994) 213, hep-th/9309110.

[117] J. Distler, S. Kachru, Singlet Couplings and (0,2) Models, Nucl. Phys. B 430 (1994) 13 , hep-th/9406090. 
[118] E. Silverstein and E. Witten, Criteria for Conformal Invariance of (0,2) Models, Nucl. Phys. B 444 (1995) 161, hep-th/9503212.

[119] R. Blumenhagen and A. Wisskirchen, Exactly Solvable (0,2) Supersymmetric String Vacua With GUT Gauge Groups, Nucl. Phys. B 454 (1995) 561, hep-th/9506104.

[120] R. Blumenhagen, R. Schimmrigk, and A. Wisskirchen, The (0,2) Exactly Solvable Structure of Chiral Rings, Landau-Ginzburg Theories and Calabi-Yau Manifolds, Nucl. Phys. B 461 (1996) 460, hep-th/9510055.

[121] S. Gukov, C. Vafa and E. Witten, CFT's From Calabi-Yau Four-folds, Nucl. Phys. B 584 (2000) 69 [Erratum-ibid. B608 (2001) 477], hep-th/9906070.

[122] S. Gukov, Solitons, Superpotentials and Calibrations, Nucl. Phys. B 574 (2000) 169, hep-th/9911011.

[123] S. Ferrara, C. Kounnas, D. Lüst, and F. Zwirner, Duality Invariant Partition Functions and Automorphic Superpotentials for $(2,2)$ String Compactifications, Nucl. Phys. B 365 (1991) 431.

[124] G. W. Moore, Attractors and Arithmetic, hep-th/9807056.

[125] G. W. Moore, Arithmetic and Attractors, hep-th/9807087.

[126] K. Behrndt, G. Lopes Cardoso, B. de Wit, R. Kallosh, D. Lüst and T. Mohaupt, Classical and Quantum $N=2$ Supersymmetric Black Holes, Nucl. Phys. B 488 (1997) 236, hep-th/9610105.

[127] F. Denef, Attractors at Weak Gravity, Nucl. Phys. B 547 (1999) 201, hep-th/9812049.

[128] P. Mayr, Summing up Open String Instantons and N=1 String Amplitudes, hepth/0203237.

[129] C. Vafa, Extending Mirror Conjecture to Calabi-Yau with Bundles, hep-th/9804131.

[130] M. Marino, R. Minasian, G. W. Moore and A. Strominger, Nonlinear Instantons from Supersymmetric p-branes, JHEP 0001 (2000) 005, hep-th/9911206.

[131] B. Körs, Open Strings In Magnetic Background Fields, Fortsch. Phys. 49 (2001) 759.

[132] M. Douglas, D-branes, Categories and N=1 Supersymmetry, hep-th/0011017.

[133] C. Römelsberger, (Fractional) Intersection Numbers, Tadpoles and Anomalies, hepth/0111086.

[134] G. Papadopoulos and P. K. Townsend, Compactification of D $=11$ Supergravity on Spaces of Exceptional Holonomy, Phys. Lett. B 357 (1995) 300, hep-th/9506150.

[135] B. S. Acharya, On Realising $N=1$ Super Yang-Mills in M theory, hep-th/0011089.

[136] E. Witten, Anomaly Cancellation On Manifolds Of $G_{2}$ Holonomy, hep-th/0108165.

[137] S. Kachru and J. McGreevy, M-theory on Manifolds of $G_{2}$ Holonomy and Type IIA Orientifolds, JHEP 0106 (2001) 027, hep-th/0103223.

[138] S. L. Shatashvili and C. Vafa, Superstrings and Manifolds of Exceptional Holonomy, hep-th/9407025.

[139] R. Blumenhagen and V. Braun, Superconformal Field Theories for Compact G Man- $_{2}$ ifolds, JHEP 0112 (2001) 006, hep-th/0110232. 


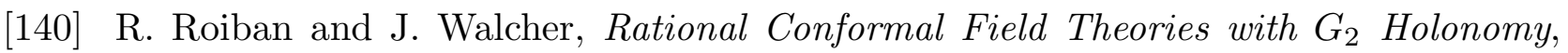
JHEP 0112 (2001) 008, hep-th/0110302.

[141] T. Eguchi and Y. Sugawara, String Theory on $G_{2}$ Manifolds based on Gepner Construction, Nucl. Phys. B 630 (2002) 132, hep-th/0111012.

[142] B. Noyvert, Unitary Minimal Models of SW(3/2,3/2,2) Superconformal Algebra and Manifolds of $G_{2}$ Holonomy, JHEP 0203 (2002) 030, hep-th/0201198.

[143] M. Faux, D. Lüst and B. A. Ovrut, Intersecting Orbifold Planes and Local Anomaly Cancellation in M-theory, Nucl. Phys. B 554 (1999) 437, hep-th/9903028.

[144] V. Kaplunovsky, J. Sonnenschein, S. Theisen and S. Yankielowicz, On the Duality between Perturbative Heterotic Orbifolds and $M$-theory on $T^{4} / \mathbb{Z}_{N}$, Nucl. Phys. B 590 (2000) 123, hep-th/9912144.

[145] M. Faux, D. Lüst and B. A. Ovrut, Local Anomaly Cancellation, M-theory Orbifolds and Phase-transitions, Nucl. Phys. B 589 (2000) 269, hep-th/0005251.

[146] M. Faux, D. Lüst and B. A. Ovrut, An M-theory Perspective on Heterotic K3 Orbifold Compactifications, hep-th/0010087.

[147] C. F. Doran, M. Faux and B. A. Ovrut, Four-dimensional $N=1$ Super Yang-Mills Theory from an $M$ theory Orbifold, hep-th/0108078.

[148] E. Gorbatov, V. S. Kaplunovsky, J. Sonnenschein, S. Theisen and S. Yankielowicz, On Heterotic Orbifolds, M-theory and Type I' Brane Engineering, JHEP 0205, 015 (2002) hep-th/0108135.

[149] M. Atiyah and E. Witten, M-theory Dynamics on a Manifold of $G_{2}$ Holonomy, hepth/0107177.

[150] R. L. Bryant and S. Salamon, On the Construction of some Complete Metrics with Exceptional Holonomy, Duke Math. J. 58 (1989) 829.

[151] G. W. Gibbons, D. N. Page and C. N. Pope, Einstein Metrics On $S^{* * 3} R^{* * 3}$ And $R^{* *} 4$ Bundles, Commun. Math. Phys. 127 (1990) 529.

[152] M. Cvetic, G. W. Gibbons, H. Lu and C. N. Pope, Cohomogeneity One Manifolds of Spin(7) and $G_{2}$ Holonomy, Phys. Rev. D 65 (2002) 106004, hep-th/0108245.

[153] B. Acharya and E. Witten, Chiral Fermions from Manifolds of $G_{2}$ Holonomy, hepth/0109152.

[154] P. Berglund and A. Brandhuber, Matter from $G_{2}$ Manifolds, hep-th/0205184.

[155] S. S. Gubser, TASI lectures: Special Holonomy in String Theory and M-theory, hepth/0201114.

[156] A. M. Uranga, Localized Instabilities at Conifolds, hep-th/0204079.

[157] C. Vafa, Superstrings and Topological Strings at Large N, J. Math. Phys. 42 (2001) 2798, hep-th/0008142.

[158] M. Atiyah, J. M. Maldacena and C. Vafa, An M-theory Flop as a Large N Duality, J. Math. Phys. 42 (2001) 3209, hep-th/0011256. 
[159] A. Brandhuber, J. Gomis, S. S. Gubser and S. Gukov, Gauge Theory at Large $N$ and New $G_{2}$ Holonomy Metrics, Nucl. Phys. B 611 (2001) 179, hep-th/0106034.

[160] A. Brandhuber, $G_{2}$ Holonomy Spaces from Invariant Three-forms, Nucl. Phys. B 629 (2002) 393, hep-th/0112113.

[161] G. Curio, B. Körs and D. Lüst, Fluxes and Branes in Type II Vacua and M-theory Geometry with $G_{2}$ and Spin(7) Holonomy, hep-th/0111165. 